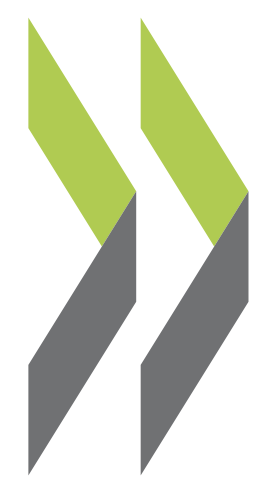

OECD Economics Department Working Papers No. 925

\title{
Less Income Inequality and More Growth - Are They Compatible? Part 2. The Distribution of Labour Income
} Isabell Koske, 
Organisation de Coopération et de Développement Économiques

Organisation for Economic Co-operation and Development

10-Jan-2012

ECONOMICS DEPARTMENT

English - Or. English

LESS INCOME INEQUALITY AND MORE GROWTH - ARE THEY COMPATIBLE?

PART 2. THE DISTRIBUTION OF LABOUR INCOME

ECONOMICS DEPARTMENT WORKING PAPER No. 925

by Isabell Koske, Jean-Marc Fournier and Isabelle Wanner

All Economics Department Working Papers are available through OECD's internet website www.oecd.org/eco/workingpapers

JT03314100

Document complet disponible sur OLIS dans son format d'origine

Complete document available on OLIS in its original format 


\section{ABSTRACT/RÉSUME \\ Less income inequality and more growth - Are they compatible? \\ Part 2. The distribution of labour income}

This paper explores the role of macroeconomic factors and structural policies in shaping the distribution of labour income. Technological change and globalisation play at least some role in driving inequality patterns, but structural policy can also have an important influence on inequality outcomes, in particular through education and labour market policies. Drawing on empirical analysis of the links between structural policies and the distribution of labour income, the paper looks at potential policy tradeoffs and complementarities with respect to the two policy objectives of lowering income inequality and raising economic growth. It concludes that many policies yield a double dividend in the sense that they contribute to achieving both goals simultaneously. This relates in particular to policies that facilitate the accumulation of human capital, that make educational achievement less dependent on personal and social circumstances, that reduce labour market dualism and that promote the labour market integration of immigrants and women.

\section{JEL classification codes: D31; F16; G18; I24; J31; J58; J71; O33}

Keywords: Income inequality; labour income; globalisation; technological change; education; labour market institutions; product market regulation

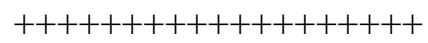

\section{Moins d'inégalités de revenu et plus de croissance - Ces deux objectifs sont-ils compatibles? Partie 2. La répartition des revenus du travail}

Ce papier explore le rôle des facteurs macroéconomiques et des politiques structurelles comme déterminants de la distribution des revenus du travail. Si les mutations technologiques et la mondialisation contribuent, à tout le moins, à la formation des inégalités, les politiques publiques, en particulier l'éducation et les politiques du marché du travail, peuvent aussi avoir une influence importante. À partir de l'analyse empirique des liens entre politiques publiques et répartition des revenus du travail, ce document examine les éventuels arbitrages et complémentarités entre les deux objectifs que sont la réduction des inégalités de revenu d'une part et le relèvement de la croissance économique d'autre part. Il conclut que nombre de politiques sont doublement payantes car elles contribuent à la réalisation simultanée de ces deux objectifs. Cela vaut en particulier pour les politiques favorisant l'accumulation de capital humain, rendant le potentiel d'éducation moins tributaire de la situation personnelle et sociale, réduisant le dualisme du marché du travail et promouvant l'intégration des immigrants et des femmes sur le marché du travail. Classification JEL : D31 ; F16 ; G18 ; I24 ; J31 ; J58 ; J71 ; O33

Mots-clés : Inégalités de revenus ; revenu du travail ; mondialisation ; mutations technologiques ; éducation ; institutions du marché du travail ; réglementation du marché de produits 


\section{TABLE OF CONTENTS}

\section{LESS INCOME INEQUALITY AND MORE GROWTH - ARE THEY COMPATIBLE?}

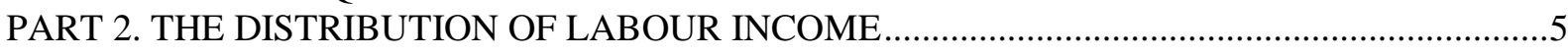

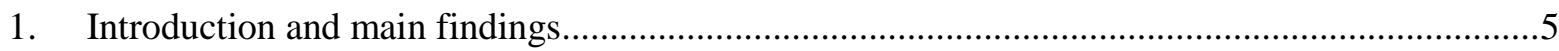

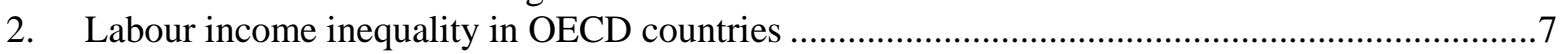

3. The role of non-policy factors in shaping labour income inequality .......................................

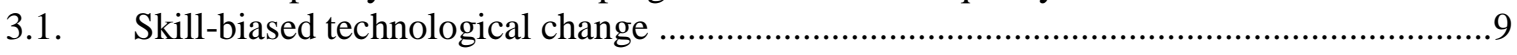

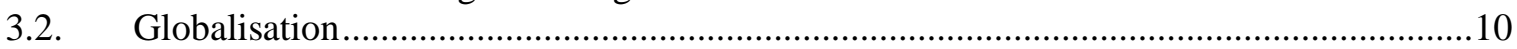

4. The role of structural policies in shaping labour income inequality........................................11

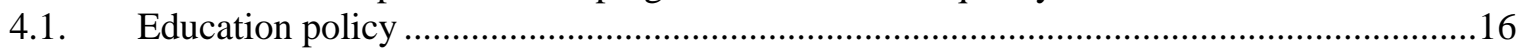

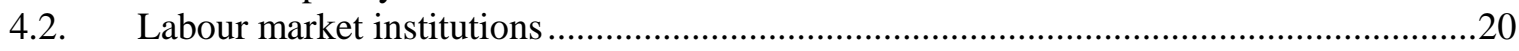

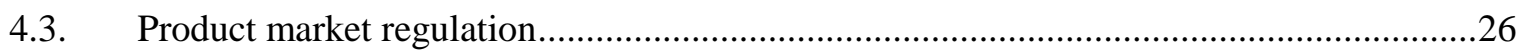

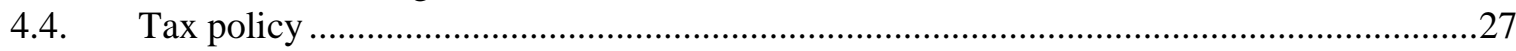

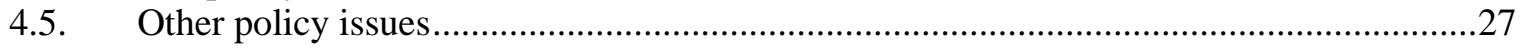

5. Identifying reform options to reduce labour income inequality ................................................. 31

6. Reducing labour income inequality and boosting GDP per capita: policy trade-offs and ..............

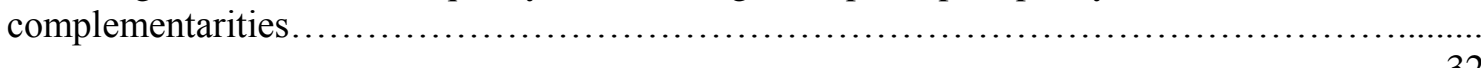

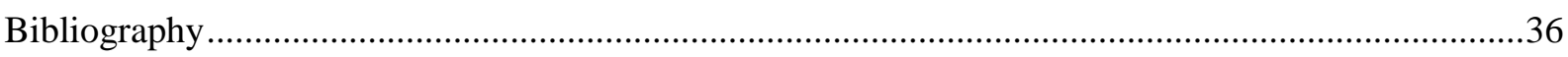

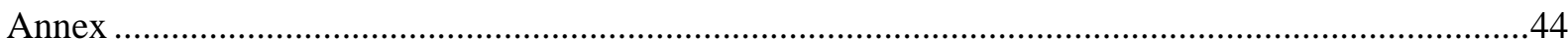

\section{Boxes}

1. The determinants of labour income inequality - a cross-country time-series analysis based on macro data

2. The determinants of labour earnings inequality - the quantile regression analysis based on micro data.

3. Explaining cross-country differences in labour earnings inequality - a decomposition based on unconditional quantile regressions.

\section{Tables}

1. Estimation results of the Bayesian model averaging analysis ................................................14

2. Results of the analysis of interaction terms for selected inequality measures .............................16

3. The impact of structural policies on labour earnings inequality ..............................................32

A1. Details on the data set used in the Bayesian model averaging analysis ......................................4

\section{Figures}

1. Labour earnings inequality .8

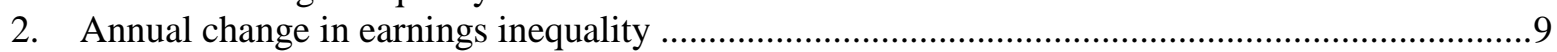

3. Employment rates of 25-64 year-olds by educational attainment ............................................17 
4. The impact of education on the distribution of earnings

5. Decomposition of cross-country differences in the logarithm of the $90 / 10$ percentile ratio.

6. Union membership - unconditional quantile regression results.............................................23

7. Earnings effect of having a temporary instead of a permanent work contract ..........................24

8. The interaction between labour market institutions and trade - wage dispersion .......................26

9. The interaction between labour market institutions and trade - employment.............................27

10. Annual change in inequality among women and men between the mid-1990s and the mid-2000s

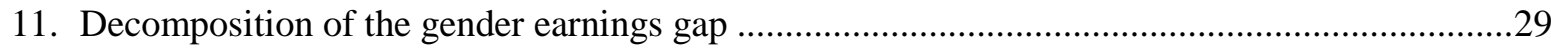

12. Decomposition of the earnings gap between natives and immigrants .......................................30

13. Indicators of policies that influence labour income inequality - the example of Canada ............31

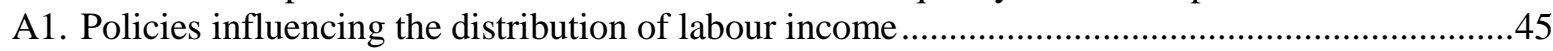

This document and any map included herein are without prejudice to the status of or sovereignty over any territory, to the delimitation of international frontiers and boundaries and to the name of any territory, city or area.

The statistical data for Israel are supplied by and under the responsibility of the relevant Israeli authorities. The use of such data by the OECD is without prejudice to the status of the Golan Heights, East Jerusalem and Israeli settlements in the West Bank under the terms of international law. 
ECO/WKP(2012)2

\title{
LESS INCOME INEQUALITY AND MORE GROWTH - ARE THEY COMPATIBLE?
}

\section{PART 2. THE DISTRIBUTION OF LABOUR INCOME}

\author{
by
}

\author{
Isabell Koske, Jean-Marc Fournier and Isabelle Wanner ${ }^{1}$
}

\section{Introduction and main findings}

Many OECD countries have seen inequality rising over the past decade. Much of this rise has reflected a widening dispersion of labour income. It seems that the benefits of economic growth have not been shared equally across all parts of the population. These developments have led to a renewed interest among researchers and policy makers in understanding the causes of labour income inequality. As the rise in inequality happened alongside rapid technological progress as well as rapidly rising trade and financial integration, much of the debate has focused on the role of technological change and globalisation in shaping the distribution of income. However, these two factors are likely to be only part of a more complex story as policy also influences labour income, both directly and indirectly by affecting the linkages with macroeconomic developments.

Against this background, this paper investigates the determinants of labour income inequality drawing on both existing and new empirical evidence. Specifically, it analyses the role of macroeconomic factors - in particular technological change and globalisation - and the role of structural policies - in particular education policy and labour and product market regulation - in shaping the distribution of labour income. While it is ultimately disposable income inequality (ideally adjusted for publicly-provided in-kind services) that matters, labour income inequality is one of its major sources and thus warrants a distinct analysis. The focus on labour income implies that important population sub-groups (such as retirees) and income sources (such as capital income) are ignored in the discussion. Moreover, tax and transfer policies are only briefly touched upon in this paper, as a thorough elaboration of their inequality effects can be found in Joumard et al. (2012), who focus on disposable rather than labour income inequality.

The following main conclusions emerge from the analysis of the drivers of labour income inequality:

- Both labour earnings inequality (inequality among those who earn an income from employment) and labour income inequality (inequality among all people in the working-age population, whether they work or not) differ widely across the OECD, reflecting crosscountry differences in wage rates, hours worked and inactivity rates.

1. The authors are members of the Economics Department of the OECD. This is one of the background papers for the OECD's project on Income Distribution and Growth-enhancing Policies. The authors would like to thank Jørgen Elmeskov, Jean-Luc Schneider, Peter Hoeller, Romain Duval, Isabelle Joumard, Mauro Pisu and Kaja Fredriksen for their useful comments and suggestions and Susan Gascard for excellent editorial support. 
- Technological change and globalisation have played a role in shaping inequality patterns, but the marked cross-country differences are also likely to reflect differences in policy and institutional settings.

- While the link between education and labour income inequality is ambiguous from a theoretical point of view, empirical evidence indicates that policies that increase upper-secondary and tertiary graduation rates help reduce inequality. Policies that promote more equal access to education (e.g. no early tracking or improving the provision of early childhood care) are also likely to reduce income inequality.

- A lower minimum wage tends to widen the earnings distribution, but the implied negative impact on labour income equality may at least partly be offset by higher employment.

- Higher union density appears to reduce labour income inequality in most countries through a more equal distribution of earnings. Although not investigated in this paper, legal extensions of collective wage agreements may have similar effects.

- Reducing labour market dualism by narrowing the gap between the protection of permanent and temporary jobs seems to lower income inequality through both a lower wage dispersion and lower unemployment.

- Several studies suggest that spending on active labour market policies may reduce income inequality by boosting employment, though the effectiveness of such measures varies widely across different types of programmes.

- The evidence on the link between product market liberalization and the dispersion of earnings is rather mixed (some studies find a positive relationship while others are unable to detect a significant link), but several studies indicate that the removal of competitionunfriendly product market regulations reduces labour income inequality by boosting employment.

- Structural policies can also mitigate particular aspects of income inequality, such as inequality between men and women. The empirical analysis carried out in this paper suggests that gender differences in working hours and choice of occupation and sector account for a sizable part of the earnings gap. Policies to reduce these differences (e.g. improvements in the access to childcare) could thus lead to more equal labour market outcomes among men and women. A large part of the gender earnings gap remains unexplained after accounting for other factors, indicating that policies that reduce gender discrimination may also help.

- Immigrants underperform relative to natives in terms of employment and wages in most countries, implying that policies that foster the integration of immigrants could reduce inequality.

Drawing on these links between structural policies and the distribution of labour income, the paper derives the following conclusions regarding possible policy trade-offs and complementarities with respect to the two policy objectives of lowering income inequality and raising economic growth:

- Many policies entail a double dividend in the sense that they reduce income inequality while at the same time boosting long-run GDP per capita. Examples include facilitating the 
accumulation of human capital, making educational potential less dependent on personal and social circumstances, reducing labour market dualism, raising spending on active labour market policies, promoting the integration of immigrants, fostering female labour market participation, avoiding gender stereotyping in education, and fighting discrimination.

- Several policies may lower income inequality at the cost of lower GDP per capita. One example are the legal extension of collective wage agreements. While the paper only explores the link between union membership and labour income inequality, the finding that higher union membership compresses the wage distribution tentatively indicates that legal extensions may have a similar effect. At the same time legal extensions may reduce economic growth by forcing firms out of the market, thus lowering competitive pressures and raising unemployment.

- For several policies that aim at boosting GDP per capita, the income inequality effects are less clear-cut. These include in particular: avoiding too high and long-lasting unemployment benefits (while in the short run such benefits are likely to reduce inequality by providing income support to the unemployed, they may raise inequality in the long run by reducing employment); liberalising product markets (the empirical evidence is inconclusive regarding the inequality impact, possibly because it depends on the nature of the reform); and lowering minimum wages (which is more likely to reduce income inequality if the cuts start from a high level).

The remainder of this paper is structured as follows. Section 2 discusses cross-country differences in both the level and recent evolution of labour income inequality. ${ }^{2}$ Section 3 then investigates the role of non-policy factors (globalisation and technological change) on the distribution of labour income, while section 4 focuses on policy factors (in particular education, labour and product market policies). Section 4 also touches upon gender inequality and migration as both affect labour income inequality. Section 5 presents country diamonds than can help to identify policy priorities for individual OECD countries and section 6 concludes with policy recommendations on how to reduce labour income inequality, also drawing attention to potential policy trade-offs or complementarities with respect to economic growth.

\section{Labour income inequality in OECD countries}

Countries differ widely with respect to the level of labour earnings inequality among individuals of working age (Figure 1). Among the OECD countries, earnings inequality for full-time employees is highest in Chile, the United States and Portugal, while Switzerland, Belgium and Denmark are the most equal countries. Inequality is generally higher for all the full-time employed, reflecting the wider dispersion of earnings among the self-employed. Extending the analysis to part-time workers, the unemployed and the inactive raises the Gini index further, reflecting the large income differentials for these groups and the group of full-time workers (unemployed individuals and the inactive enter the calculation with zero income as transfers are not taken into account). The increase in the Gini index is particularly large for countries where part-time workers make up a sizable share of total employment (e.g. Australia, Germany and the Netherlands), and for countries with a high unemployment rate and many inactive people in the working-age population. While the Gini indices of the population subgroups are highly correlated (the correlation coefficients are between 0.8 and 0.9 ), there are several countries for which the choice of the group matters considerably for the inequality ranking.

2. The focus on labour income inequality implies a focus on the working age population, meaning that retirees and children are ignored. Whenever subgroups of the working-age population are referred to (for example, when discussing specific empirical results) this is explicitly stated in the text. 
Figure 1. Labour earnings inequality

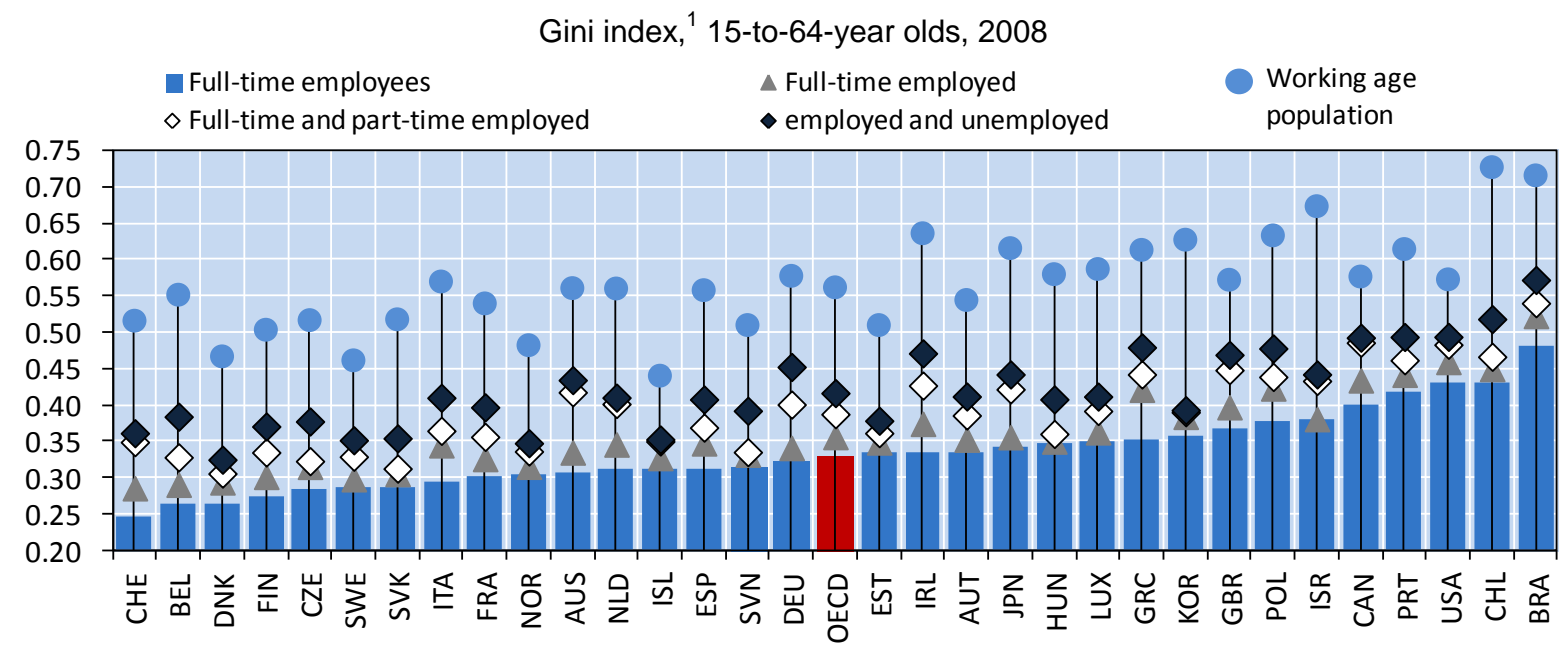

1. The Gini index ranges from zero (perfect equality) to one (perfect inequality).

Note: The group of employed individuals includes both dependent and self-employed individuals. The working age population includes all persons aged 15 to 64 except for students and persons above the country's statutory retirement age. The Gini coefficients take into account labour earnings only; the precise definition of labour earnings differs across countries. 2005 for Israel, 2006 for Brazil, 2007 for France, Korea and the United States, 2009 for Chile and Japan. The values for the OECD are calculated as unweighted averages across all OECD countries for which data are available.

Source: Panel Study of Income Dynamics (PSID) for the United States; Household Income and Labour Dynamics in Australia Survey (HILDA) for Australia; National Socioeconomic Characterization Survey for Chile; Korean Labour and Income Panel Study (KLIPS) for Korea; Luxembourg Income Study (LIS) for Brazil and Israel; Japan Household Panel Survey (JHPS) for Japan, Swiss Household Panel (SHP) for Switzerland, and European Union Statistics on Income and Living Conditions (EU-SILC) for the other countries.

The evolution of inequality among the full-time employed also differed widely. While many OECD countries saw a marked rise in inequality over the past decade, it remained broadly unchanged or even declined in others (Figure 2). ${ }^{3}$ In about half of the countries that experienced an increase in labour earnings inequality between the mid-1990s and the mid-2000s, it was driven by rising inequality in the upper half of the earnings distribution. In the remaining countries, the increase in inequality was more broad-based, affecting all parts of the earnings distribution. Only in Germany was rising inequality due to increasing inequality at the bottom half of the earnings distribution. In the majority of countries, the widening of the earnings distribution was accompanied by a rise in employment, the only exceptions being the Czech Republic, Poland and the United States.

3. Diverging trends in working hours may account for part of the differences in the evolution of labour earnings, but the size of this effect is difficult to gauge. 
Figure 2. Annual change in labour income inequality

Percentage change in 90/10 percentile ratio and percentage point change in employment rate Full-time employees, mid-1990s to mid-2000s

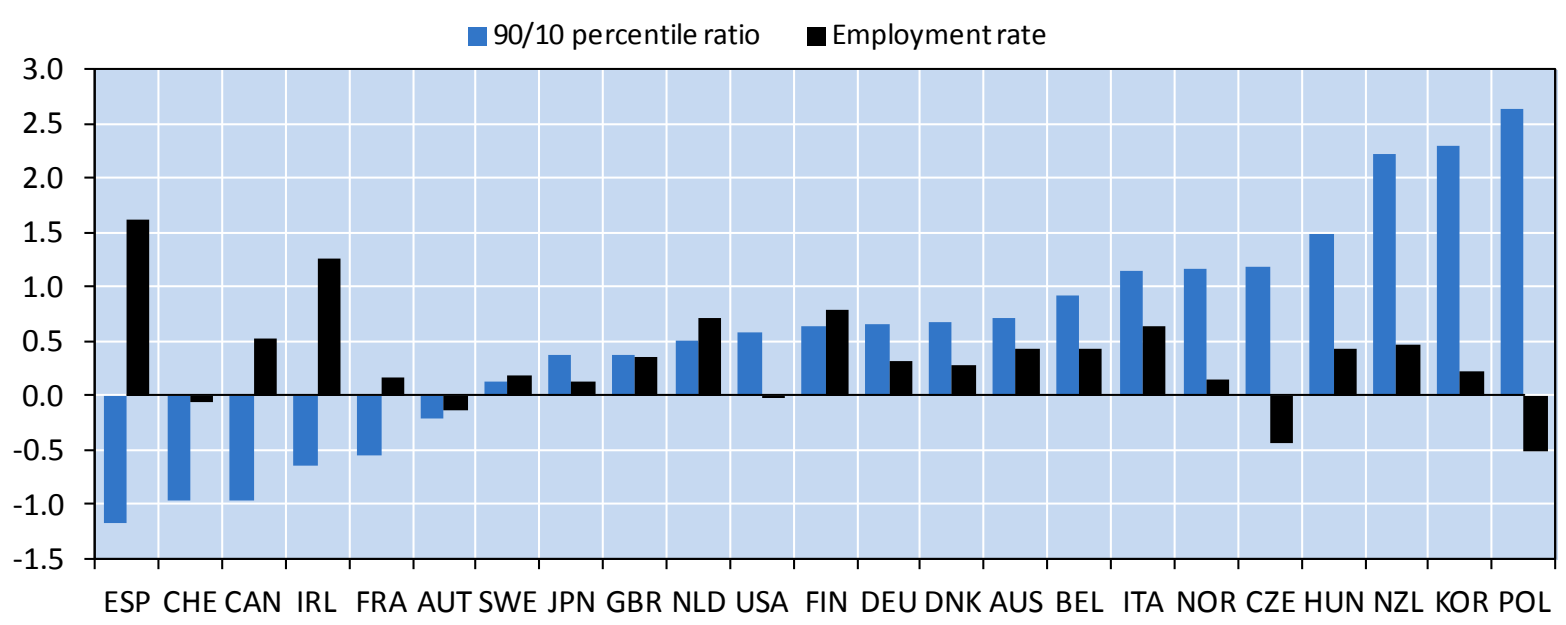

Note: For the employment rate the annual change between 1995 and 2005 is shown. For the percentile ratio, the mid-1990s refer to 1993 for Belgium, to 1994 for Canada, to 1996 for Denmark and the Czech Republic, to 1997 for Ireland and Norway, and to 1995 for all other countries; the mid-2000s refer to 2004 for Poland and to 2005 for all other countries.

Source: National household and enterprise surveys.

\section{The role of non-policy factors in shaping labour income inequality}

\subsection{Skill-biased technological change}

One prominent explanation for the rise in labour income inequality is skill-biased technological change. In its (more recent) nuanced version (Acemoglu and Autor, 2010), this theory assumes that highly-skilled workers have an advantage in performing non-routine abstract tasks while medium-skilled workers have an advantage in performing routine tasks that are based on precise and well-understood procedures, while low-skilled workers have an advantage in performing non-routine manual tasks. ${ }^{4}$ To the extent that computers substitute for routine tasks, they reduce the demand for medium-skilled workers, while that for the high-skilled and (possibly) low-skilled rises. If the demand shifts are not offset by equal shifts in supply, technological progress reduces the earnings or employment of medium-skilled workers relative to both the low- and high-skilled ones. The effect on the earnings of high-skilled relative to low-skilled workers is a priori ambiguous, but it is likely to be positive, because laid-off medium-skilled workers move down in the task distribution and depress the earnings of the low skilled so that the relative earnings of high skilled workers rise. ${ }^{5}$

4. The (older) canonical view (see, for example, Tinbergen, 1974, 1975; Katz and Murphy, 1992) focused instead on high and low-skilled workers, with technological progress raising the productivity and thus wages more for the high than the low skilled. While this view was successful in explaining wage developments in the United States between the 1960s and 1980s, it fails to explain more recent developments, in particular the polarisation of wages and employment.

5. Performance pay is one channel through which changes in returns to skill, due e.g. to technological progress, translate into higher wage inequality. Lemieux et al. (2009) show that a rise in the demand for skills induces more firms to offer performance-pay contracts (because the market returns to effort 
The implications of this hypothesis for wage and employment patterns have found empirical support for many OECD countries. The past decades have seen a polarisation of employment by skill level (e.g. Autor et al., 2006, Goos et al., 2009). In particular, labour demand seems to have shifted from routine to non-routine tasks, with the shift concentrated in those industries that computerised rapidly (Autor et al., 2003). It is more difficult to find support at the macro level, possibly reflecting problems in identifying the right group of workers and in properly capturing technological change. While new cross-country time-series evidence suggests that technological change has contributed to a rise in earnings inequality, the choice of specification matters for the significance and size of the effect and also for where in the earnings distribution the impact is most pronounced (see Box 1, Table 1 and OECD, 2011a).

\subsection{Globalisation}

While globalisation has many facets, the debate on the link between globalisation and labour income inequality has mostly focused on trade, migration (which is discussed below) and, to a lesser extent, investment flows. Early empirical studies were based on the Stolper-Samuelson theorem, which predicts that trade increases the real return for the factor with which a country is most abundantly endowed. However, the theorem proved to be inconsistent with the evidence and in particular, the observed rise in inequality in low-income countries (e.g. Goldberg and Pavcnik, 2007; Feenstra, 2008; Stone and Cavazos, 2011). Several new mechanisms have thus been explored (see Harrison et al., 2010 for an overview). ${ }^{6}$ For the most part, they predict that labour income inequality should increase with globalisation:

- Offshoring: Feenstra and Hanson (1996) explain the simultaneous rise in labour income inequality in both rich and poor countries by the reallocation of tasks from the richer to the poorer ones. The tasks that are reallocated are typically not skill intensive from the perspective of the skill-rich country, but they are from the perspective of the skill-poor country. As a result, offshoring makes labour demand more skill intensive in both groups of countries. Empirical studies tend to confirm that offshoring from high to low-wage countries leads to a rise in inequality in the source country, though there is disagreement about the magnitude of this effect. ${ }^{7}$ Workers performing routine tasks appear to suffer the most from offshoring (Ebenstein et al., 2009).

- Firm heterogeneity: If firms differ in their profitability and the least productive firms are battered by import competition, trade may increase labour income inequality by lowering employment or by reducing the relative earnings of low-income workers if high-income workers work disproportionately in (high-productivity) exporting firms (e.g. Egger and Kreickemeier, 2009; Helpman et al., 2010). ${ }^{8}$ Empirical studies support the thrust of these theoretical models,

and thus the benefits of implementing performance-pay systems increase) and results in more wage inequality among workers with performance-related pay.

6. Globalisation may also influence consumption inequality. For example, if cheap imports from low-wage countries are disproportionately consumed by low-income households, globalisation should lower inequality (e.g. Broda and Romalis, 2009).

7. Liu and Trefler (2008), for example, conclude that the effects are at best small, while Ebenstein et al. (2009), who also account for occupational exposure to globalisation, find sizable negative effects.

8. Note that any change in employment has direct repercussions for the dispersion of earnings, with the direction of the effect depending on which individuals lose their job. If it is low-income workers (which is likely to be the case here), the decline in employment is mirrored by a compression of earnings among those who remain employed. 
showing for example that trade liberalisation is associated with an increase in the wages of workers at multinational enterprises and a decline in wages at domestically-oriented firms (Amiti and Davis, 2008).

- Trade-induced innovation: By spurring innovation, trade may raise labour income inequality both temporarily - since the R\&D process is skill intensive (Dinopoulos and Segerstrom, 1999; Neary, 2003) - and permanently provided it results in skill-biased technological change (Acemoglu, 2002). There is evidence that firms that begin exporting after trade liberalisation upgrade technology and workers' skills more quickly (Bustos, 2007).

While the positive link between globalisation and inequality is supported by a growing body of firm-level studies, it is more difficult to establish a robust link between globalisation and inequality at the aggregate level. ${ }^{9}$ Cross-country time-series regressions suggest that trade increases the dispersion of earnings only if unions are weak or if employment protection is lax, whereas the negative employment effects of trade are more pronounced under the presence of strong unions (see Figures 8 and 9 and the discussion below). However, these results should be interpreted with great care given the many limitations of the analysis (Box 1). The impact of cross-border financial flows that may for instance be associated with offshoring appears to depend on their direction. A greater stock of external assets is found to be associated with a more equal earnings distribution in OECD countries by raising the relative earnings of those at the bottom. Since financial outflows from OECD countries are still dominated by flows towards other OECD economies rather than emerging markets, this finding is in line with earlier evidence showing that offshoring to high-income locations raises the wages of routine workers (e.g. Ebenstein et al., 2009). By contrast, external liabilities do not seem to have a strong impact on the distribution of earnings, though there is some evidence that they boost employment, possibly because FDI inflows boost labour demand.

\section{The role of structural policies in shaping labour income inequality}

This section discusses the impact of education, labour and product market policies on labour income inequality both from a theoretical and empirical perspective. Tax policy is only briefly touched upon since a thorough discussion of its inequality effects can be found in Joumard et al. (2012). The empirical discussion draws on new cross-country time-series regressions (Box 1) as well as existing literature, in particular the study by Fournier and Koske (2012) which estimates quantile regressions based on household survey data for 31 different countries (the methodology used in this study is briefly summarized in Box 2). The quantile regressions do not allow investigating as many policies as the cross-country time-series regressions, but they have the important advantage of relying on large household survey datasets, so that the results are more reliable. The discussion of gender and migration issues draws on existing literature as well as Oaxaca-Blinder decompositions based on household survey data.

9. While some studies confirm that trade raises inequality (e.g. Richardson, 1995; Wood, 1995) others draw the opposite conclusion (e.g. Checchi and García-Peñalosa, 2010) or are unable to establish a significant link (e.g. Edwards, 1997; OECD, 2011a). 


\section{Box 1. The determinants of labour income inequality - a cross-country time-series analysis}

Building on recent OECD work (OECD, 2011a), the determinants of labour income inequality are explored by estimating a series of reduced-form equations for 22 OECD countries ${ }^{1}$ that relate different measures of income inequality $Y$ to measures of globalisation $G L O B$, technological change $T E C H$, human capital $H C$, policy indicators $P$ and a set of control variables $X$ :

$$
Y_{i t}=\alpha G L O B_{i t}+\beta T E C H_{i t}+\gamma H C_{i t}+\delta P_{i t}+\phi X_{i t}+\mu_{i}+\lambda_{t}+\varepsilon_{i t}
$$

where $i$ and $t$ denote country and time. All equations include country and period fixed effects to control for unobserved cross-country heterogeneity and unobserved shocks that affect all countries simultaneously. Equation (1) is estimated for several dependent variables: $I$ ) the 90/10, 90/50 and 50/10 percentile ratios for full-time employees to capture the dispersion of wages among the employed; ii) the employment rate; and iii) the Gini index to capture the combined impact. As time-series data for the Gini index for labour income are not available for a wide range of countries, the Gini indices are based on disposable income and are calculated over the whole population (not just the working-age population). The estimated coefficients in the Gini regressions thus capture more than just the impact of the explanatory variables on labour income inequality (for example, the coefficient on the unemployment benefit replacement rate captures both the indirect effects whereby a higher replacement rate may reduce employment as well as the dispersion of wages and the direct effect whereby it raises the income of the unemployed).

Three measures of globalisation are used: total trade (exports plus imports), foreign assets and foreign liabilities (all expressed as a ratio to GDP). Technical change is measured by the number of patents per one million inhabitants. The stock of human capital is captured by the share of the population with post-secondary education. The policy indicators span four different areas: labour market policy (measured by the overall level of employment protection and the level of protection on regular and temporary work, the unemployment benefit replacement rate and the ratio of the minimum to the median wage), product market regulation (measured by an index of regulation in seven non-manufacturing industries), financial market regulation (measured by a financial reform index), and taxation (measured by the labour tax wedge). Moreover, union density is included to investigate the role of wage setting mechanisms. The set of control variables includes the output gap and, except for the employment rate specifications, the female employment share and employment in agriculture and industry (both as a share of total employment). Details on the dataset are provided in the Annex.

Taking a macroeconomic perspective allows exploiting a broad range of reform experiences simultaneously and capturing the full general equilibrium effects of the explanatory variables. However, such cross-country time-series regressions are typically less robust to variations in, for example, samples, econometric methods or the set of variables included in the regression than analysis based on micro data. In order to deal with the model uncertainty problem, the Bayesian model averaging (BMA) technique proposed by Sala-i-Martin et al. (2004) - which the authors call Bayesian Averaging of Classical Estimates - is used. The method essentially constructs estimates as a weighted average of ordinary least square estimates for every possible combination of the included variables, where the weights applied to individual regressions are justified on Bayesian grounds. The results of the exercise are summarised in Tables 1 and $2 .^{2}$

Since institutions may influence inequality not only directly but also indirectly by shaping the impact of non-institutional drivers, additional equations are estimated that interact the policy variables with the measures of globalisation and technological change. The equations are estimated using a standard fixed-effects approach. Following standard practice, the interactions are specified as multiplicative terms, which take the form of products of deviations of policies and macroeconomic variables from their sample means:

$$
\begin{aligned}
Y_{i t}= & \alpha G L O B_{i t}+\beta T E C H_{i t}+\gamma H C_{i t}+\delta P_{i t}+\phi X_{i t}+\mu_{i}+\lambda_{t} \\
& +\omega\left(G L O B_{i t}-G L O B^{*}\right)\left(P_{i t}-P^{*}\right)+\tau\left(T E C H_{i t}-T E C H^{*}\right)\left(P_{i t}-P^{*}\right)+\varepsilon_{i t}
\end{aligned}
$$

$G L O B^{*}, T E C H^{*}$ and $P^{*}$ are the sample means (across countries and over time) of GLOB, TECH and $P$, respectively, and the other variables are denoted as before. Since no robust evidence could be found for an interaction between policies and, respectively, technological change and financial globalisation, the final results are based on a specification that only interacts the trade-to-GDP ratio with the policy indicators. The regression results are summarised in Figures 8 and 9, and Table 3.

1. Australia, Austria, Belgium, Canada, Czech Republic, Denmark, Finland, France, Germany, Hungary, Ireland, Italy, Japan, Netherlands, New Zealand, Norway, Poland, Spain, Sweden, Switzerland, United Kingdom, United States.

2. For the most part, the BMA technique used here confirms the conclusions drawn in OECD (2011a). The main exceptions are product market regulation, for which OECD (2011a) finds a significant negative link with wage dispersion and financial assets/liabilities for which it finds a significant positive/negative link (in contrast to the analysis here, the focus of OECD, 2011a, is on foreign direct investment). 


\section{Box 2. The determinants of labour earnings inequality - a quantile regression analysis}

Fournier and Koske (2012) investigate the determinants of labour earnings inequality using household survey data from 32 countries. ${ }^{1}$ The authors consider all individuals aged between 15 and 64 who are working either part-time or full-time and earn a positive income during the reference year. ${ }^{2}$ For each country, they estimate several equations that relate the logarithm of an individual's labour income to different sets of explanatory variables. In the baseline model, the explanatory variables include the logarithm of the individual's number of working hours, the gender, the age and age squared and dummy variables for the education level. ${ }^{3}$ Several additional variables are of interest but are excluded from the authors' baseline equation because they exist only for a subset of countries or pose potential endogeneity problems. These are dummy variables for the sector of work and the occupation, the number of years of work experience, and dummy variables for having a temporary as opposed to a permanent work contract, for being self-employed, for being member of a union, for working in the public sector, for having foreign citizenship, for being born in a foreign country, and for having obtained a PhD. These variables are added to the baseline equation in a series of alternative specifications.

While standard least squares techniques could be used to study the relationship between the explanatory variables and labour earnings, they would only provide an estimate of the effect on the mean. To draw conclusions about the impact on earnings inequality it is necessary to go beyond the mean and estimate the effect of the explanatory variables also on other parts of the earnings distribution. Fournier and Koske (2012) do this using the unconditional quantile regression technique proposed by Firpo et al. (2007a, 2009). ${ }^{4}$ The method allows investigating the marginal impact of certain characteristics (such as the level of education) on different parts of the earnings distribution such as the median or the $10^{\text {th }}$ or $90^{\text {th }}$ centile. The procedure is implemented by simultaneously estimating nine quantile regressions, one for each decile in the range 0.1 to 0.9 . For each year and country, the regression thus does not yield a single coefficient, but nine different coefficients, one for each decile.

By comparing the different coefficients, it is possible to draw conclusions about the impact of a change in a certain variable on earnings inequality. For example, if a rise in the education level of the workforce has a positive impact on the earnings of all individuals, but the impact is larger for those at the bottom of the earnings distribution (i.e. for lower deciles) than for those at the top (i.e. for higher deciles), this means that a rise in the education level of the workforce is associated with a fall in earnings inequality. It is important to note that the method is static in nature, thus abstracting from general equilibrium effects. This implies in particular that it accounts only for changes in the composition of the workforce in terms of certain characteristics, ignoring that such changes may alter the relative returns to these characteristics (for example, a rise in the share of uppersecondary graduates among the working-age population may reduce the relative returns to upper-secondary degrees).

1. Austria, Belgium, the Czech Republic, Denmark, Estonia, Finland, France, Germany, Greece, Hungary, Iceland, Ireland, Italy, Luxembourg, the Netherlands, Norway, Poland, Portugal, Slovak Republic, Slovenia, Spain, Sweden and the United Kingdom (European Union Statistics on Income and Living Conditions); Australia (Household Income and Labour Dynamics in Australia Survey); Canada (Survey of Labour and Income Dynamics); Chile (National Socioeconomic Characterization Survey); Korea (Korean Labour and Income Panel); Japan (Japan Household Panel Survey); Switzerland (Swiss Household Panel); the United States (Panel Study of Income Dynamics); Brazil and Israel (Luxembourg Income Study).

2. Individuals with zero or negative income are excluded from the analysis (although rare in the datasets, negative incomes may occur if self-employed individuals make a loss).

3. The level of education is captured by two dummy variables, the first one being equal to unity for all individuals who have at least finished upper-secondary education, and hence also takes value one for those who have a tertiary degree. The second one being equal to unity for all individual who have finished tertiary education. Hence, the coefficient on the first dummy gives the impact of an upper-secondary or post-secondary non-tertiary education relative lower-secondary education or less and the coefficient on the second dummy gives the impact of tertiary education relative to uppersecondary or post-secondary non-tertiary education.

4. Since this technique is still fairly new, the more established approach by Koenker and Bassett (1978) is also used by Fournier and Koske (2012). However, because this approach does not allow estimating the impact on overall earnings inequality of changes in the explanatory variables, the focus of the present paper is on the method by Firpo et al. (2007a, 2009). 
ECO/WKP(2012)2

Table 1. Estimation results of the Bayesian model averaging analysis

\begin{tabular}{|c|c|c|c|c|c|c|c|c|c|c|c|c|c|c|c|}
\hline \multirow{2}{*}{$\begin{array}{c}\text { Dependent variable } \\
\text { Trade (as share of GDP) }\end{array}$} & \multicolumn{3}{|c|}{ 90/10 percentile ratio } & \multicolumn{3}{|c|}{ 90/50 percentile ratio } & \multicolumn{3}{|c|}{$50 / 10$ percentile ratio } & \multicolumn{3}{|c|}{ Gini index } & \multicolumn{3}{|c|}{ Emplovment rate } \\
\hline & $\begin{array}{c}0.026 \\
(0.028) \\
{[0.135]}\end{array}$ & $\begin{array}{c}0.050 \\
(0.036) \\
{[0.169]}\end{array}$ & $\begin{array}{c}0.004 \\
(0.031) \\
{[0.102]}\end{array}$ & $\begin{array}{l}-0.001 \\
(0.002) \\
{[0.026]}\end{array}$ & $\begin{array}{l}-0.001 \\
(0.002) \\
0.025]\end{array}$ & $\begin{array}{c}0.166 \\
(0.064) \\
{[0.553]}\end{array}$ & $\begin{array}{c}0.030 \\
(0.022) \\
{[0.225]}\end{array}$ & $\begin{array}{c}0.164 \\
(0.061) \\
{[0.713]}\end{array}$ & $\begin{array}{l}-0.285 \\
(0.115) \\
{[0.750]}\end{array}$ & $\begin{array}{l}-0.002 \\
(0.003) \\
{[0.133]}\end{array}$ & $\begin{array}{l}-0.003 \\
(0.003) \\
{[0.110]}\end{array}$ & $\begin{array}{l}-0.100 \\
(0.027) \\
0.991]\end{array}$ & $\begin{array}{l}-0.025 \\
(0.012) \\
{[0.402]}\end{array}$ & $\begin{array}{l}-0.099 \\
(0.029) \\
0.967]\end{array}$ & $\begin{array}{l}-0.120 \\
(0.030) \\
{[0.999]}\end{array}$ \\
\hline Foreign assets (as share of GDP) & $\begin{array}{l}-0.087 \\
(0.025) \\
{[0.977]}\end{array}$ & $\begin{array}{l}{[0.128]} \\
-0.128 \\
(0.027) \\
{[0.998]}\end{array}$ & $\begin{array}{c}{[0.102]} \\
0.026 \\
(0.013) \\
{[0.455]}\end{array}$ & $\begin{array}{l}{[0.001} \\
(0.000) \\
{[0.063]}\end{array}$ & $\begin{array}{l}{[0.0<0]} \\
-0.001 \\
(0.000) \\
{[0.057]}\end{array}$ & $\begin{array}{c}{[0.000} \\
0.000 \\
(0.001) \\
{[0.062]}\end{array}$ & $\begin{array}{l}-0.013 \\
(0.006) \\
{[0.651]}\end{array}$ & $\begin{array}{l}{[0.045} \\
(0.007) \\
{[0.999]}\end{array}$ & $\begin{array}{c}{[0.019} \\
(0.010) \\
{[0.573]}\end{array}$ & $\begin{array}{l}-0.020 \\
(0.004) \\
{[1.000]}\end{array}$ & $\begin{array}{l}-0.011 \\
(0.002) \\
{[0.995]}\end{array}$ & $\begin{array}{c}{[.049} \\
0.049 \\
(0.008) \\
{[1.000]}\end{array}$ & $\begin{array}{c}{[0.40<]} \\
0.000 \\
(0.001) \\
{[0.113]}\end{array}$ & $\begin{array}{c}0.003 \\
0.001) \\
{[0.315]}\end{array}$ & $\begin{array}{l}-0.023 \\
(0.007) \\
{[0.981]}\end{array}$ \\
\hline $\begin{array}{l}\text { Foreign liabilities (as share of } \\
\text { GDP) }\end{array}$ & $\begin{array}{c}0.037 \\
(0.018) \\
{[0.494]}\end{array}$ & $\begin{array}{c}0.049 \\
(0.020) \\
{[0.560]}\end{array}$ & $\begin{array}{c}0.004 \\
(0.010) \\
{[0.282]}\end{array}$ & $\begin{array}{c}0.000 \\
(0.000) \\
{[0.045]}\end{array}$ & $\begin{array}{c}0.000 \\
(0.000) \\
{[0.041]}\end{array}$ & $\begin{array}{c}0.000 \\
(0.001) \\
{[0.063]}\end{array}$ & $\begin{array}{c}0.001 \\
(0.003) \\
{[0.243]}\end{array}$ & $\begin{array}{c}0.005 \\
(0.003) \\
{[0.214]}\end{array}$ & $\begin{array}{c}0.003 \\
(0.008) \\
{[0.380]}\end{array}$ & $\begin{array}{c}0.011 \\
(0.004) \\
{[0.792]}\end{array}$ & $\begin{array}{c}0.002 \\
(0.001) \\
{[0.274]}\end{array}$ & $\begin{array}{l}-0.054 \\
(0.008) \\
1.000]\end{array}$ & $\begin{array}{c}0.012 \\
(0.002) \\
{[0.979]}\end{array}$ & $\begin{array}{c}0.010 \\
(0.002) \\
{[0.800]}\end{array}$ & $\begin{array}{c}0.029 \\
(0.008) \\
{[0.993]}\end{array}$ \\
\hline Patents (per 1 million inhabitants) & $\begin{array}{c}0.035 \\
(0.011) \\
{[0.866]}\end{array}$ & $\begin{array}{c}0.026 \\
(0.009) \\
{[0.681]}\end{array}$ & $\begin{array}{l}-0.015 \\
(0.009) \\
{[0.318]}\end{array}$ & $\begin{array}{l}-0.006 \\
(0.002) \\
{[0.422]}\end{array}$ & $\begin{array}{l}-0.006 \\
(0.002) \\
{[0.447]}\end{array}$ & $\begin{array}{l}0.001 \\
(0.001) \\
{[0.094]}\end{array}$ & $\begin{array}{l}0.033 \\
(0.006) \\
{[1.000]}\end{array}$ & $\begin{array}{c}0.029 \\
(0.005) \\
{[1.000]}\end{array}$ & $\begin{array}{l}-0.006 \\
(0.004) \\
{[0.326]}\end{array}$ & $\begin{array}{l}0.000 \\
(0.000) \\
{[0.130]}\end{array}$ & $\begin{array}{c}0.000 \\
(0.000) \\
{[0.093]}\end{array}$ & $\begin{array}{l}0.001 \\
(0.001) \\
{[0.226]}\end{array}$ & $\begin{array}{l}-0.022 \\
(0.002) \\
{[1.000]}\end{array}$ & $\begin{array}{l}-0.020 \\
(0.002) \\
{[1.000]}\end{array}$ & $\begin{array}{l}-0.024 \\
(0.002) \\
{[1.000]}\end{array}$ \\
\hline $\begin{array}{l}\text { Share of population with post- } \\
\text { secondary education }\end{array}$ & $\begin{array}{l}-1.094 \\
(0.300) \\
{[0.963]}\end{array}$ & $\begin{array}{l}-0.440 \\
(0.173) \\
{[0.528]}\end{array}$ & $\begin{array}{l}-0.341 \\
(0.155) \\
{[0.404]}\end{array}$ & $\begin{array}{l}-0.440 \\
(0.090) \\
{[0.960]}\end{array}$ & $\begin{array}{l}-0.444 \\
(0.091) \\
{[0.965]}\end{array}$ & $\begin{array}{c}0.000 \\
(0.009) \\
{[0.069]}\end{array}$ & $\begin{array}{l}-0.600 \\
(0.134) \\
{[0.995]}\end{array}$ & $\begin{array}{l}-0.031 \\
(0.021) \\
{[0.156]}\end{array}$ & $\begin{array}{l}-0.294 \\
(0.120) \\
{[0.610]}\end{array}$ & $\begin{array}{c}0.030 \\
(0.016) \\
{[0.403]}\end{array}$ & $\begin{array}{c}0.002 \\
(0.003) \\
{[0.077]}\end{array}$ & $\begin{array}{c}0.005 \\
(0.009) \\
{[0.172]}\end{array}$ & $\begin{array}{c}0.000 \\
(0.003) \\
{[0.070]}\end{array}$ & $\begin{array}{l}-0.014 \\
(0.011) \\
{[0.217]}\end{array}$ & $\begin{array}{c}0.009 \\
(0.020) \\
{[0.370]}\end{array}$ \\
\hline Employment protection legislation & $\begin{array}{r}-10.252 \\
(2.495) \\
{[0.987]}\end{array}$ & & $\begin{array}{c}0.529 \\
(0.615) \\
{[0.145]}\end{array}$ & $\begin{array}{c}0.116 \\
(0.067) \\
{[0.073]}\end{array}$ & & $\begin{array}{l}1.590 \\
(0.604) \\
{[0.462]}\end{array}$ & $\begin{array}{l}-8.889 \\
(1.168) \\
{[1.000]}\end{array}$ & & $\begin{array}{l}-0.223 \\
(0.413) \\
{[0.193]}\end{array}$ & $\begin{array}{l}-0.984 \\
(0.296) \\
{[0.835]}\end{array}$ & & $\begin{array}{l}-0.039 \\
(0.069) \\
{[0.157]}\end{array}$ & $\begin{array}{c}0.001 \\
(0.027) \\
{[0.071]}\end{array}$ & & $\begin{array}{l}-1.372 \\
(0.454) \\
{[0.979]}\end{array}$ \\
\hline $\begin{array}{l}\text { Employment protection on regular } \\
\text { work }\end{array}$ & & $\begin{array}{c}19.729 \\
(5.650) \\
{[0.891]}\end{array}$ & & & $\begin{array}{l}-0.318 \\
(0.174) \\
{[0.081]}\end{array}$ & & & $\begin{array}{c}19.507 \\
(2.100) \\
{[1.000]}\end{array}$ & & & $\begin{array}{l}-2.866 \\
(0.519) \\
{[0.999]}\end{array}$ & & & $\begin{array}{l}-2.898 \\
(0.612) \\
{[0.997]}\end{array}$ & \\
\hline $\begin{array}{l}\text { Employment protection on } \\
\text { temporary work }\end{array}$ & & $\begin{array}{l}-5.868 \\
(1.230) \\
{[0.999]}\end{array}$ & & & $\begin{array}{c}0.139 \\
(0.065) \\
{[0.138]}\end{array}$ & & & $\begin{array}{l}-5.151 \\
(0.482) \\
{[1.000]}\end{array}$ & & & $\begin{array}{l}-0.107 \\
(0.048) \\
{[0.276]}\end{array}$ & & & $\begin{array}{c}0.006 \\
(0.023) \\
{[0.118]}\end{array}$ & \\
\hline Union density (in \%) & $\begin{array}{l}-0.692 \\
(0.208) \\
{[0.936]}\end{array}$ & $\begin{array}{l}-0.125 \\
(0.062) \\
{[0.261]}\end{array}$ & $\begin{array}{l}-0.653 \\
(0.197) \\
{[0.880]}\end{array}$ & $\begin{array}{l}-0.002 \\
(0.002) \\
{[0.030]}\end{array}$ & $\begin{array}{l}-0.003 \\
(0.003) \\
{[0.031]}\end{array}$ & $\begin{array}{l}-0.100 \\
(0.037) \\
{[0.469]}\end{array}$ & $\begin{array}{l}-0.448 \\
(0.101) \\
{[0.999]}\end{array}$ & $\begin{array}{l}-0.006 \\
(0.008) \\
{[0.079]}\end{array}$ & $\begin{array}{l}-0.173 \\
(0.074) \\
{[0.632]}\end{array}$ & $\begin{array}{l}-0.070 \\
(0.023) \\
{[0.787]}\end{array}$ & $\begin{array}{l}-0.116 \\
(0.030) \\
{[0.982]}\end{array}$ & $\begin{array}{l}-0.144 \\
(0.028) \\
{[1.000]}\end{array}$ & $\begin{array}{l}-0.004 \\
(0.004) \\
{[0.112]}\end{array}$ & $\begin{array}{l}-0.111 \\
(0.036) \\
{[0.927]}\end{array}$ & $\begin{array}{c}0.132 \\
(0.029) \\
{[1.000]}\end{array}$ \\
\hline $\begin{array}{l}\text { Unemployment benefit } \\
\text { replacement rate }\end{array}$ & $\begin{array}{c}0.492 \\
(0.165) \\
{[0.885]}\end{array}$ & $\begin{array}{c}0.334 \\
(0.123) \\
{[0.665]}\end{array}$ & $\begin{array}{c}0.010 \\
(0.035) \\
{[0.108]}\end{array}$ & $\begin{array}{c}0.009 \\
(0.005) \\
{[0.073]}\end{array}$ & $\begin{array}{c}0.011 \\
(0.006) \\
0.086]\end{array}$ & $\begin{array}{c}0.096 \\
(0.043) \\
{[0.372]}\end{array}$ & $\begin{array}{c}0.065 \\
(0.034) \\
{[0.390]}\end{array}$ & $\begin{array}{c}0.006 \\
(0.006) \\
{[0.088}\end{array}$ & $\begin{array}{l}-0.086 \\
(0.059) \\
{[0.341]}\end{array}$ & $\begin{array}{l}-0.154 \\
(0.021) \\
{[1.000]}\end{array}$ & $\begin{array}{l}-0.127 \\
(0.019) \\
{[1.000]}\end{array}$ & $\begin{array}{c}0.009 \\
(0.009) \\
{[0.231]}\end{array}$ & $\begin{array}{l}-0.188 \\
(0.025) \\
{[1.000]}\end{array}$ & $\begin{array}{l}-0.179 \\
(0.024) \\
{[1.000]}\end{array}$ & $\begin{array}{l}-0.172 \\
(0.040) \\
{[1.000]}\end{array}$ \\
\hline $\begin{array}{l}\text { Minimum wage (as ratio to } \\
\text { median wage) }\end{array}$ & & & $\begin{array}{l}-2.487 \\
(0.369) \\
{[1.000]}\end{array}$ & & & $\begin{array}{l}-0.014 \\
(0.014) \\
{[0.099]}\end{array}$ & & & $\begin{array}{l}-1.057 \\
(0.182) \\
{[1.000]}\end{array}$ & & & $\begin{array}{l}-0.193 \\
(0.039) \\
{[1.000]}\end{array}$ & & & $\begin{array}{l}-0.006 \\
(0.015) \\
{[0.361]}\end{array}$ \\
\hline Tax wedge (in \%) & $\begin{array}{l}-1.827 \\
(0.297) \\
{[1.000]}\end{array}$ & $\begin{array}{l}-2.264 \\
(0.294) \\
{[1.000}\end{array}$ & $\begin{array}{l}-0.158 \\
(0.103) \\
{[0.268]}\end{array}$ & $\begin{array}{l}-0.171 \\
(0.056) \\
{[0.5771}\end{array}$ & $\begin{array}{l}-0.157 \\
(0.052) \\
{[0.536]}\end{array}$ & $\begin{array}{c}0.022 \\
(0.016) \\
{[0.126]}\end{array}$ & $\begin{array}{l}-0.617 \\
(0.129) \\
{[1.000]}\end{array}$ & $\begin{array}{l}-1.083 \\
(0.117) \\
{[1.000}\end{array}$ & $\begin{array}{l}-0.390 \\
(0.150) \\
{[0.770]}\end{array}$ & $\begin{array}{c}0.008 \\
(0.008) \\
{[0.190]}\end{array}$ & $\begin{array}{c}0.006 \\
(0.005) \\
{[0.126]}\end{array}$ & $\begin{array}{c}0.030 \\
(0.018) \\
0.356]\end{array}$ & $\begin{array}{l}-0.146 \\
(0.042) \\
{[0.9271}\end{array}$ & $\begin{array}{l}-0.096 \\
(0.035) \\
{[0.763]}\end{array}$ & $\begin{array}{l}-0.137 \\
(0.047) \\
{[0.974]}\end{array}$ \\
\hline $\begin{array}{l}\text { Product market regulation } \\
\text { indicator }\end{array}$ & $\begin{array}{l}-0.035 \\
(0.210) \\
0.107]\end{array}$ & $\begin{array}{c}0.188 \\
(0.221) \\
{[0.114]}\end{array}$ & $\begin{array}{c}7.824 \\
(2.212) \\
{[0.958]}\end{array}$ & $\begin{array}{c}0.174 \\
(0.082) \\
{[0.121]}\end{array}$ & $\begin{array}{c}0.169 \\
(0.080) \\
{[0.117]}\end{array}$ & $\begin{array}{l}2.271 \\
(0.600) \\
{[0.829]}\end{array}$ & $\begin{array}{l}-1.047 \\
(0.473) \\
{[0.533]}\end{array}$ & $\begin{array}{l}-0.098 \\
(0.087) \\
{[0.108]}\end{array}$ & $\begin{array}{c}0.248 \\
(0.278) \\
{[0.217]}\end{array}$ & $\begin{array}{c}0.658 \\
(0.202) \\
{[0.830]}\end{array}$ & $\begin{array}{c}0.170 \\
(0.072) \\
{[0.313]}\end{array}$ & $\begin{array}{l}1.412 \\
(0.239) \\
{[1.000]}\end{array}$ & $\begin{array}{c}0.020 \\
(0.025) \\
{[0.096]}\end{array}$ & $\begin{array}{c}0.010 \\
(0.031) \\
{[0.122]}\end{array}$ & $\begin{array}{l}-0.520 \\
(0.228) \\
{[0.870]}\end{array}$ \\
\hline Financial reform index & $\begin{array}{c}0.009 \\
(0.057) \\
{[0.102]}\end{array}$ & $\begin{array}{l}-0.002 \\
(0.046) \\
{[0.084]}\end{array}$ & $\begin{array}{l}-3.335 \\
(0.670) \\
{[0.998]}\end{array}$ & $\begin{array}{c}0.004 \\
(0.005) \\
{[0.026]}\end{array}$ & $\begin{array}{c}0.004 \\
(0.005) \\
{[0.026]}\end{array}$ & $\begin{array}{l}-0.027 \\
(0.024) \\
{[0.093]}\end{array}$ & $\begin{array}{c}0.003 \\
(0.029) \\
{[0.114]}\end{array}$ & $\begin{array}{l}-0.001 \\
(0.015) \\
{[0.063]}\end{array}$ & $\begin{array}{l}-1.247 \\
(0.358) \\
{[0.962]}\end{array}$ & $\begin{array}{c}{[0.312} \\
(0.074) \\
{[0.989]}\end{array}$ & $\begin{array}{c}0.250 \\
(0.066) \\
{[0.971]}\end{array}$ & $\begin{array}{c}1.0 .260 \\
(0.080) \\
{[0.924]}\end{array}$ & $\begin{array}{c}0.202 \\
(0.070) \\
{[0.786]}\end{array}$ & $\begin{array}{c}0.131 \\
0.131 \\
(0.055) \\
{[0.615]}\end{array}$ & $\begin{array}{l}-0.410 \\
(0.097) \\
{[1.000]}\end{array}$ \\
\hline Number of observations & 311 & 311 & 172 & 311 & 311 & 172 & 311 & 311 & 172 & 356 & 356 & 177 & 385 & 385 & 203 \\
\hline Prior mean model size & 7.5 & 8.0 & 8.0 & 7.5 & 8.0 & 8.0 & 7.5 & 8.0 & 8.0 & 7.5 & 8.0 & 8.0 & 6.0 & 6.5 & 6.5 \\
\hline Posterior mean model size & 9.1 & 8.4 & 8.7 & 2.6 & 2.7 & 5.7 & 9.2 & 7.8 & 10.7 & 9.9 & 7.8 & 10.5 & 6.6 & 8.8 & 11.5 \\
\hline
\end{tabular}


Table 2. Gauging the relative importance of the drivers of income inequality based on the BMA analysis

Impact of a one-standard-deviation change in the explanatory variable on the dependent variable (in ppt)

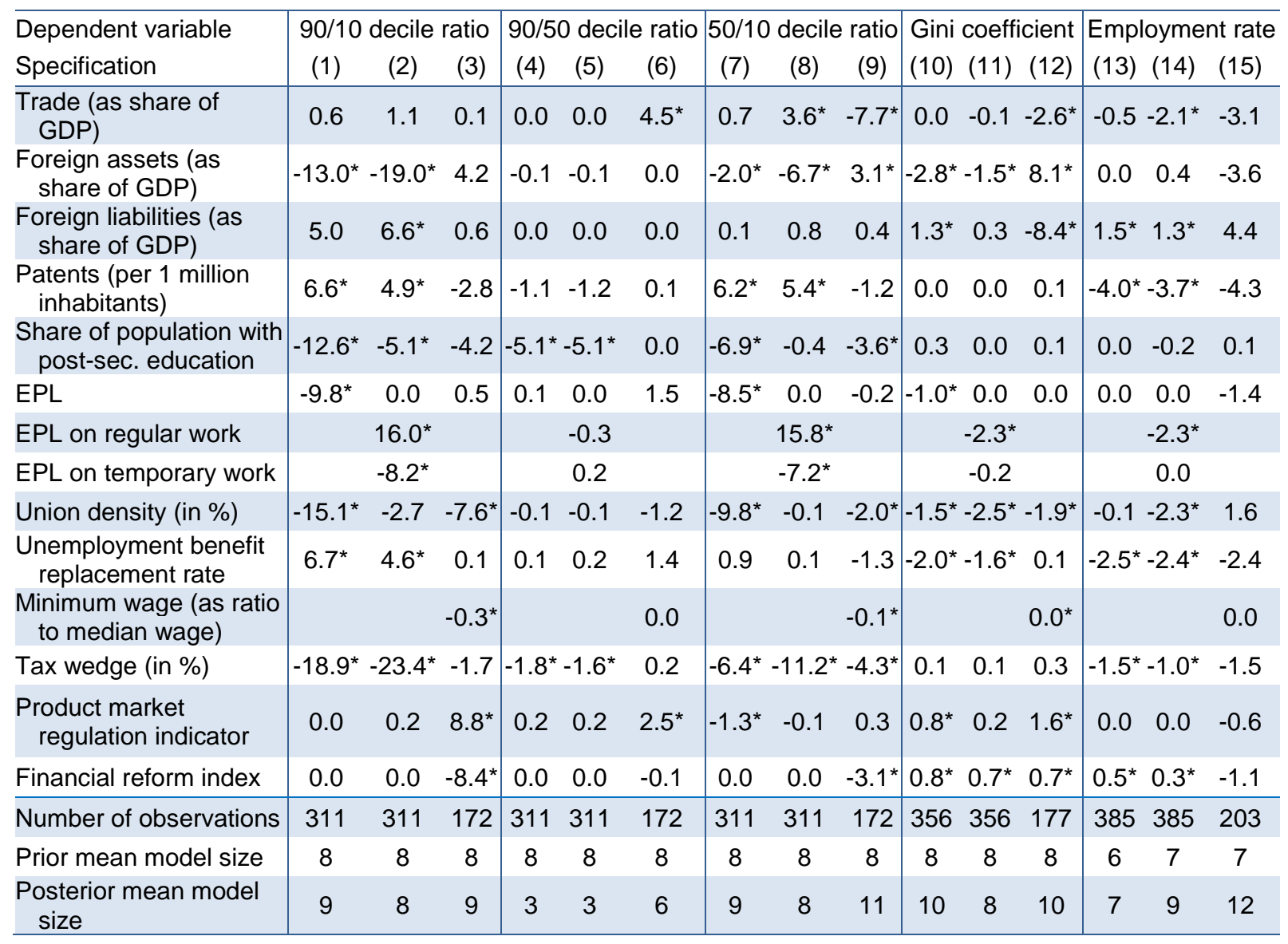

Note: The one-standard-deviation change in the explanatory variables is calculated across all countries that are included in the regression and across all years between 1981 and 2007 for which data are available. An asterisk means that the inclusion probability of the variable is above $50 \%$. 
Table 3. Results of the analysis of interaction terms for selected inequality measures

\begin{tabular}{|c|c|c|c|}
\hline Dependent variable & $90 / 10$ decile ratio & $90 / 10$ decile ratio & Employment rate \\
\hline Trade (as share of GDP) & $\begin{array}{l}0.575^{\star \star \star} \\
(0.209)\end{array}$ & $\begin{array}{l}0.659^{\star * \star} \\
(0.188)\end{array}$ & $\begin{array}{l}-12.880^{\star \star \star} \\
(2.622)\end{array}$ \\
\hline Foreign assets (as share of GDP) & $\begin{array}{l}-0.195^{\star \star *} \\
(0.044)\end{array}$ & $\begin{array}{l}-0.149^{* * *} \\
(0.042)\end{array}$ & $\begin{array}{l}-0.022 \\
(0.543)\end{array}$ \\
\hline Foreign liabilities (as share of GDP) & $\begin{array}{l}0.179^{* * *} \\
(0.043)\end{array}$ & $\begin{array}{l}0.135^{\star \star *} \\
(0.042)\end{array}$ & $\begin{array}{l}1.056^{*} \\
(0.561)\end{array}$ \\
\hline Patents (per 1 million inhabitants) & $\begin{array}{l}-0.002 \\
(0.015)\end{array}$ & $\begin{array}{l}0.037^{* *} \\
(0.015)\end{array}$ & $\begin{array}{l}-0.925^{\star \star *} \\
(0.201)\end{array}$ \\
\hline $\begin{array}{l}\text { Share of population with post-secondary } \\
\text { education }\end{array}$ & $\begin{array}{l}-1.846^{* * *} \\
(0.343)\end{array}$ & $\begin{array}{l}-1.180^{* * *} \\
(0.326)\end{array}$ & $\begin{array}{l}10.569^{* *} \\
(4.868)\end{array}$ \\
\hline Employment protection legislation & $\begin{array}{l}-10.845^{\star * *} \\
(3.016)\end{array}$ & & \\
\hline Employment protection legislation * trade & $\begin{array}{l}-0.354^{\star * *} \\
(0.130)\end{array}$ & & \\
\hline Union density (in \%) & & $\begin{array}{l}-0.821^{* * *} \\
(0.173)\end{array}$ & $\begin{array}{l}-3.396 \\
(2.560)\end{array}$ \\
\hline Union density * trade & & $\begin{array}{l}-0.013^{* * *} \\
(0.005)\end{array}$ & $\begin{array}{l}-0.488^{\star * *} \\
(0.070)\end{array}$ \\
\hline Number of cross sections & 22 & 22 & 22 \\
\hline Number of observations & 377 & 420 & 516 \\
\hline Adjusted R-squared & 0.97 & 0.96 & 0.92 \\
\hline
\end{tabular}

\subsection{Education policy}

Theory suggests that the link between education and labour income inequality is far from straightforward. The impact of a change in the educational composition of the workforce can have two separate effects (Knight and Sabot, 1983): i) a composition effect, whereby a rise in the share of highly-educated (high-wage) workers raises earnings inequality up to a certain point, but will then lower it as fewer low-education (low-wage) workers remain; ${ }^{10}$ and $i$ ) a compression rate-of-return effect, whereby a rise in the share of highly-educated workers alters the returns to education. ${ }^{11}$

Given these theoretical ambiguities, the impact of education - and, hence, education policy - on labour income inequality is an empirical issue. Owing to the lack of a comprehensive dataset on education policy indicators, the empirical literature has focused on the inequality effects of education outcomes rather than policies. ${ }^{12}$ In general, the better educated are found to benefit from higher employment rates and

10. Specifically, while the higher dispersion of wages among highly-educated individuals means that an increase in their share raises earnings inequality, a second inverted-U-shaped effect is superimposed on this monotonic first effect, whereby a rise in the share of highly-educated individuals initially raises earnings inequality as some of the workers now earn the higher wage that is associated with a higher education level, but eventually inequality declines as more and more individuals earn this higher wage (Robinson, 1976).

11. The direction of the change in (relative) returns depends on many factors, in particular the substitutability or complementarity between low- and highly-educated workers..

12. The main exception is education spending. However, the empirical results are mixed, possibly because spending alone does not take into account the country-specific institutional setup and because it is a poor measure of the quality of the education system. For example, Sylwester (2002) concludes that higher public spending on education is associated with lower earnings inequality, while Checchi (2000) draws the 
higher earnings than less educated individuals (Figure 3 and OECD, 2010a). Similarly, workplace training appears to boost wages, though there is uncertainty regarding the magnitude of this effect (e.g. Goux and Maurin, 2000, Arulampalam and Booth, 2001; Leuven and Oosterbeek, 2002). ${ }^{13}$

Figure 3. Employment rates of 25-64 year-olds by educational attainment

Number of 25-64 year-olds in employment as a percentage of the population aged 25 to 64, 2008

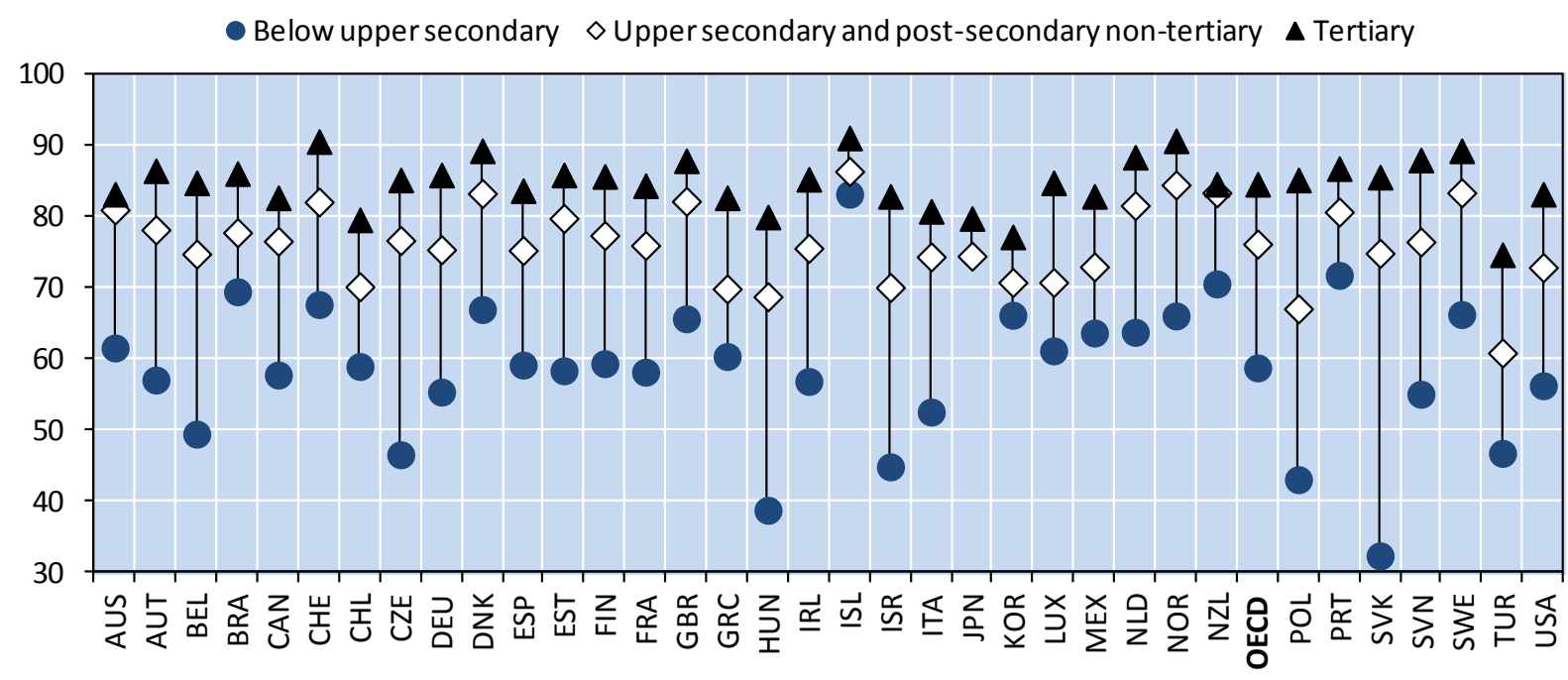

Source: OECD (2010), Education at a Glance 2010, Table A6.3a.

The quantile regressions presented in Fournier and Koske (2012) allow investigating the impact of a general rise in the educational attainment of the workforce on the level of earnings inequality. They show that through a pure composition effect the dispersion of earnings falls as more individuals go beyond lower-secondary school and acquire upper-secondary degrees - a result that is to be expected as the majority of individuals in the countries considered already have upper-secondary education (Figure 4, Panel A). ${ }^{14} \mathrm{~A}$ rise in the number of tertiary graduates, by contrast, raises the dispersion of earnings (Figure 4, Panel B). ${ }^{15}$ These composition effects may be strengthened or weakened by changes in the returns to education that result from changes in the relative supply of low- and high-educated workers, which are not taken into account by the quantile regressions, which are static in nature. As regards tertiary education, cross-country time-series analysis (which account for both the composition and the rate-ofreturn effects) tentatively indicates that a rise in the number of tertiary graduates may lower the relative returns to tertiary degrees enough to more than offset the composition effect, so that a rise in tertiary

opposite conclusion. Hoxby (2008) looks specifically at redistributive school spending and finds for the United States that it reduces earnings inequality among adults, but that the effect is very small.

13. Private returns to training tend to be higher for better educated workers, which may explain their more intensive participation in training (Bassanini et al., 2005).

14. Two notable exceptions are Portugal and Brazil, where upper secondary education is found to be more profitable for those at the top of the earnings distribution. This could be due to the lower average education level compared with the other countries in the sample. The results for Portugal are in line with existing empirical evidence (e.g. Machado and Mata, 2001; Hartog et al., 2001).

15. Increasing the share of tertiary graduates should influence income inequality also by raising the number of low-income students. This effect is not captured by the empirical analysis. 
graduation rates is associated with lower earnings inequality (Table 1 and OECD, 2011a) ${ }^{16}$ However, this result needs to be taken with great care given the fragility of the underlying empirical framework. A decomposition of cross-country differences in earnings inequality suggests that differences in the educational composition of the workforce play an important role (see Box 3).

\section{Figure 4. The impact of education on the distribution of earnings}

Panel A. Effect on log earnings of raising the number of upper-secondary or post-secondary non-tertiary graduates

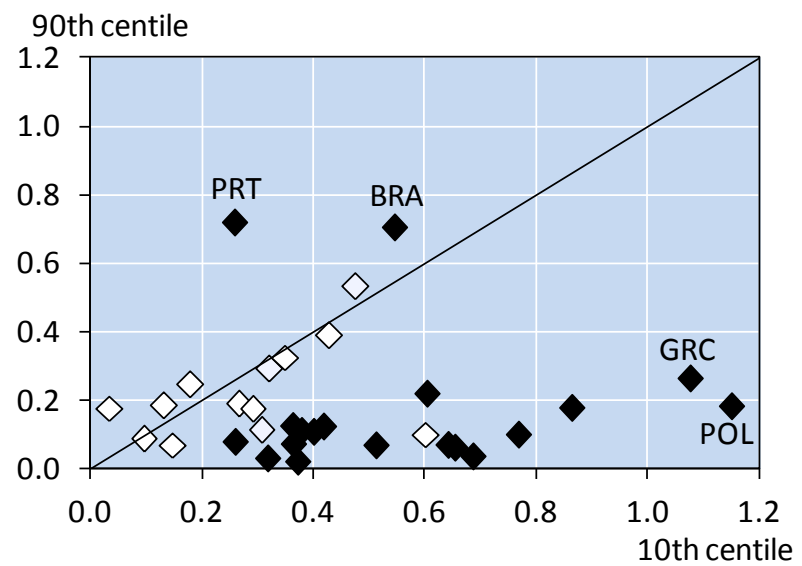

Panel B. Effect on log earnings of raising the number of tertiary graduates

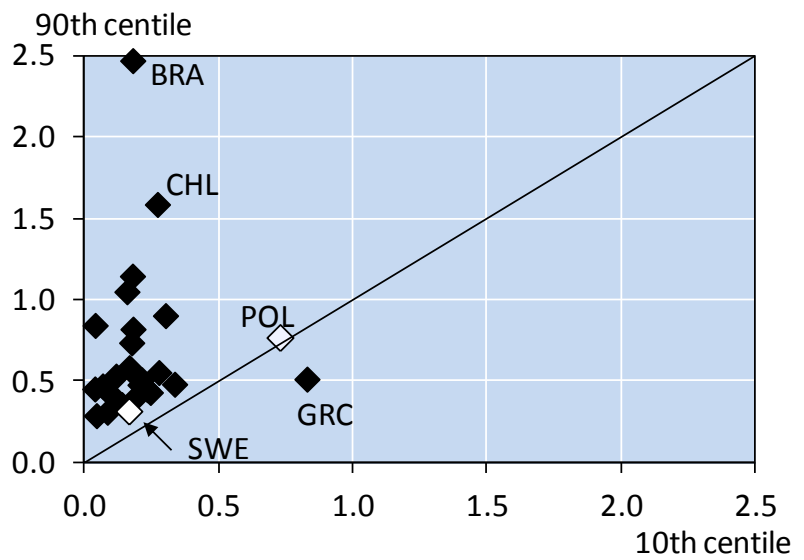

- Wage effect differs significantly $\diamond$ Wage effect is the same at the 10th and 90th centiles

Note: The horizontal axis shows the impact of a $1 \%$ increase in the proportion of workers with respectively uppersecondary or post-secondary non-tertiary education (Panel A) and tertiary education (Panel B) on the log earnings of the $10^{\text {th }}$ quantile. The vertical axis shows the impact of the same change on the log earnings of the $90^{\text {th }}$ quantile. A data point below (above) the 45 degree line indicates that the change in the educational composition of the workforce is associated with a fall (rise) in earnings inequality.

Source: Fournier, J.M. and I. Koske (2012), "Less Income Inequality and More Growth - Are they Compatible? Part 7. The Drivers of Labour Earnings Inequality - An Analysis Based on Conditional and Unconditional Quantile Regressions", OECD Economics Department Working Papers, No. 930.

The importance of acquiring knowledge for labour market outcomes has increased over the last couple of decades. An upper-secondary degree is today typically considered the minimum needed to find a decent job (OECD, 2010a). ${ }^{17}$ Moreover, while the returns to lower-secondary education have declined over time, those to post-secondary education have surged (e.g. Lemieux, 2006b; Machado and Mata, 2001), most likely reflecting a rise in the demand for skills that has outpaced the rise in supply. ${ }^{18}$ Among workers

16. This implies that the return to tertiary education falls with a rise in the share of individuals who hold a tertiary degree. A simple panel regression analysis that regresses the average rate of return to a tertiary degree on the share of employed and unemployed individuals holding such a degree and country-fixed effects lends support to this negative relationship. For upper-secondary and post-secondary non-tertiary degrees by contrast, the regression does not provide any evidence for a significant link between the returns to such degrees and the share of workers holding them, suggesting that the composition effect estimated with the quantile regressions can be regarded as the total effect. See Fournier and Koske (2012) for details.

17. Upper secondary education (ISCED level 3) includes both general and (pre-)vocational/(pre-)technical education.

18. Specifically, while the rise in the supply of workers with post-secondary education is likely to have put downward pressure on the returns to post-secondary degrees, this effect was more than offset by an opposite effect stemming from a rise in demand associated with skill-biased technological change. 
with post-secondary education, relative wage gains have been higher for those higher up in the wage distribution.

Both theoretical and empirical studies suggest that greater equality in educational attainment - measured, for example, by the variance of the number of years of schooling - lowers earnings inequality (e.g. De Gregorio and Lee, 2002). This is supported by the quantile regressions of Fournier and Koske (2012), which show that the returns to education differ across education levels (Figure 4). Policies to promote equity in education by ensuring a basic minimum standard of education for all and by limiting the influence of personal and social circumstances on educational potential should thus also be beneficial for earnings equality.

\section{Box 3. Explaining cross-country differences in labour earnings inequality - a decomposition based on unconditional quantile regressions}

There are many ways to decompose cross-country differences in labour earnings inequality (see, for example, the survey by Fortin et al., 2011). Building on unconditional quantile regressions, Fournier and Koske (2012) use a methodology close to the one proposed by Firpo et al. (2007b) to decompose cross-country differences into: $i)$ differences in the composition of the workforce (for example, inequality should be higher in countries with a more unequal distribution of education endowment) and ii) differences in rates of return (for example, inequality should be higher in countries with a larger wage gap between high- and low-educated workers).

The measure of labour earnings inequality used for the decomposition is the $90 / 10$ percentile ratio calculated over full-time and part-time workers with positive earnings during the reference year. The United States is used as reference country so that each country's 90/10 percentile ratio is compared with the 90/10 percentile ratio of the United States. For each explanatory variable, the composition effect is constructed from a comparison of the variable's mean in the two countries. ${ }^{2}$ The rate-of-return effect is computed by running two separate unconditional quantile regressions, one for the United States and another one for the country of interest, and then comparing the coefficients for the $10^{\text {th }}$ and $90^{\text {th }}$ percentiles obtained from the two regressions. ${ }^{3,4}$

The following findings emerge from this decomposition (Figure 5 and Fournier and Koske, 2012):

- Differences in the rates of return to personal characteristics such as the level of education, the age or hours worked account for a large part of the gap in the 90/10 percentile ratio between OECD countries.

- While cross-country differences in the composition of the workforce in terms of the education level, hours worked or the sector of employment also play a role, the effects of these differences on crosscountry differences in labour earnings inequality roughly offset each other in most cases.

- Factors such as the share of public sector employment, the share of financial sector employment or the age structure of the workforce do not play a big role in shaping differences in earnings inequality within the OECD. 
Figure 5. Decomposition of cross-country differences in the logarithm of the $90 / 10$ percentile ratio

United States is used as the reference country, $2007^{1}$

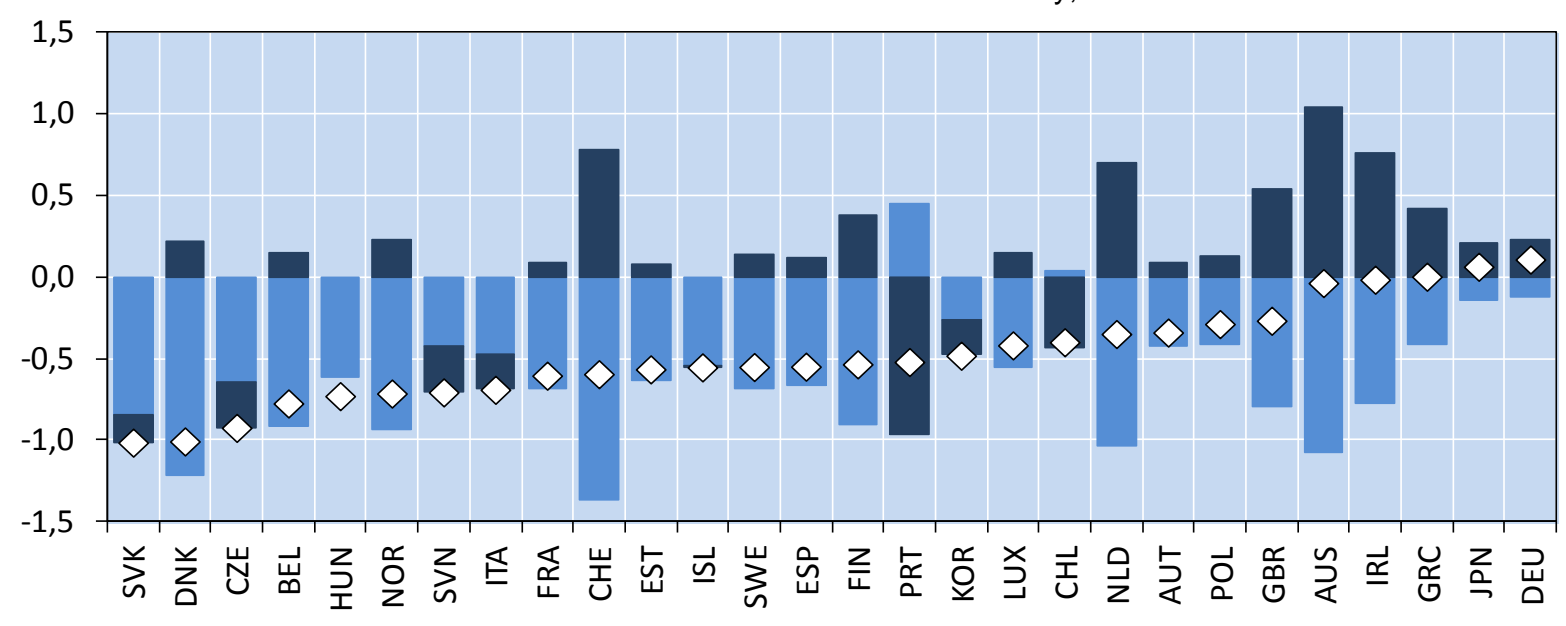

Sum of rate of return effects plus contribution of the constant $\square$ Sum of composition effects

$\diamond$ Total difference in the $90 / 10$ percentile ratio ${ }^{2}$

1. 2008 for Canada; 2009 for Chile and Japan.

2. 90/10 percentile ratio of the country shown on the horizontal axis minus 90/10 percentile ratio of the United States.

Source: Fournier, J.M. and I. Koske (2012), "Less Income Inequality and More Growth - Are They Compatible? Part 7. The Drivers of Labour Earnings Inequality - An Analysis Based on Conditional and Unconditional Quantile Regressions", OECD Economics Department Working Papers, No. 930.

1. Although the results depend somewhat on the choice of the reference country, the general conclusions are fairly robust to this choice.

2. While focusing on the mean is a strong simplification for continuous variables such as hours worked, the mean contains all information in the case of dummy variables.

3. The methodology by Firpo et al. (2007b) makes use of a third regression that is run on the country of interest and assumes that all explanatory variables follow the same distribution in that country as they do in the United States. This third regression is omitted in the approach adopted in this paper and the information is instead taken from the regression on the country of interest without changing the distribution of the explanatory variables. This simpler approach assumes that the probability to be above a certain quantile in the distribution is linear in the set of explanatory variables.

4. The precise magnitude of the rate-of-return effect needs to be interpreted with caution as it also reflects the uncertainty that is attached to the unconditional quantile regression estimates.

\section{2. $\quad$ Labour market institutions}

Labour market institutions affect labour income inequality through their (possibly conflicting) impacts on relative earnings and employment: ${ }^{19}$

- Minimum wages: A minimum wage set above the market-clearing level can raise unemployment and thus inequality by lowering labour demand. ${ }^{20}$ At the same time, a rise in the minimum wage may reduce the dispersion of wages among those who remain employed (by raising the wages of those at

19. While the discussion of the employment effects focuses mostly on the unemployed, similar arguments apply to those that use disability or early retirement schemes as substitutes for unemployment.

20. If firms have monopsony power, a rise in the minimum wage above the equilibrium wage reduces the monopsonistic rent of employers, thereby increasing employment until the minimum wage reaches the wage level that would prevail in a perfectly competitive labour market. Further increases in the minimum wage beyond this level will reduce employment (e.g. Bassanini and Duval, 2006). 
the bottom of the wage distribution), thereby contributing to a decline in inequality. ${ }^{21}$ The latter effect is confirmed by a large number of empirical studies (e.g. Di Nardo et al., 1996; Lee, 1999; and Lemieux, $2006 a$ for the United States; Dickens et al., 1999, for the United Kingdom; Koeniger et al., 2007 and Checchi and García-Peñalosa, 2010 for panels of OECD countries) as well as the crosscountry time-series analysis conducted in this study (Table 1). However, the literature is less conclusive regarding employment: while some studies, including the present one cannot detect a link between minimum wages and overall employment, others find negative employment effects for at least some types of workers such as the very low-skilled (see Neumark and Wascher, 2007 for a recent review). ${ }^{22}$ Although this issue is unsettled, the few studies that looked at the combined effect conclude that an increase in minimum wages from their current level would generally raise inequality (as measured by the Gini index), as unemployment increases and this dominates the effect of a more compressed wage distribution (e.g. Calderón et al., 2005; Checchi and García-Peñalosa, 2008).

- Wage bargaining: In theory, the influence of the wage bargaining system on wage inequality is ambiguous and depends on $i$ ) the number of workers who are covered by collective agreements (be it through union membership or through administrative extensions of collective agreements), ii) the strength of unions as manifested in their wage effects (in terms of the difference between union and non-union members of the level and the dispersion of wages) and iii) the level at which bargaining takes place (e.g. at the firm, industry or economy-wide level). ${ }^{23}$ Regarding the first effect, the new cross-country time-series analysis conducted in this study (Table 1) suggests that, on average across countries, a rise in the share of workers affiliated to a trade union is associated with lower wage inequality among full-time workers - consistent with earlier evidence (e.g. Kahn, 2000; Burniaux et al., 2006). The quantile regressions by Fournier and Koske (20112), which are more robust and also account for the second effect (i.e. the strength of unions), confirm this conclusion for some, but not all countries (Figure 6): higher union membership is found to decrease the dispersion of wages in Australia, Canada, Switzerland and the United States, but to have no effect in Korea and favours middle-income earners in Chile and Japan. Studies that account for legal extensions by looking at union coverage instead of union membership also point to wage equalising effects (e.g. OECD, 2011a, Kahn, 2000), though legal extensions appear to be detrimental to employment (Murtin et al., 2012). Regarding the third effect, i.e. the level of bargaining, several studies show that highly centralised/coordinated wage bargaining systems deliver better employment outcomes than decentralised/uncoordinated or intermediate systems (e.g. Bassanini and Duval, 2006). ${ }^{24}$ Moreover, the wage compression effect of unionisation appears to be smaller if wage bargaining is more

21. There are two effects: a direct one, which is the elimination of the left tail of the wage distribution, and an indirect, general equilibrium one that leads to changes in relative wages as firms substitute the laid-off workers with workers of slightly higher productivity (Teulings, 2003).

22. This may reflect different effects of minimum wages of different levels.

23. The relationship between the number of workers covered by collective agreements and economy-wide earnings inequality is inverted U-shaped with the shape of the curve depending on the relative means and variances of the wages of union and non-union workers (the wages of union workers tend to be higher on average than those of non-union workers and less dispersed; see Gosling and Machin, 1995, Machin, 1997, and Fournier and Koske, 2012). The strength of unions and the level of bargaining influence the shape of the curve by affecting the average level and/or the dispersion of union members' wages.

24. The literature on the relationship between the level of bargaining and employment is, however, far from being conclusive (Flanagan, 1999). Some studies even indicate a humped-shaped relationship so that intermediate systems based on branch-level bargaining yield the worst labour market outcomes (e.g. Elmeskov et al., 1998; Calmfors and Driffill, 1988). 
coordinated, possibly because unions take the adverse employment effects of wages that are pushed beyond the market-clearing level better into account (Koeniger et al., 2007).

- Employment protection legislation (EPL): If firms incur costs when dismissing workers, they will be reluctant to hire, thereby lowering labour turnover. The impact of EPL on employment is thus theoretically ambiguous and also the empirical literature is inconclusive (Bassanini and Duval, 2006). Empirical evidence on the impact of EPL on the earnings distribution is scarce. The crosscountry time-series regressions carried out in OECD (2011a) as well as in the present study (Table 1) tentatively indicate that strict EPL narrows the dispersion of wages among full-time employees, ${ }^{25}$ thereby confirming the results of Koeniger et al. (2007). The substantial fixed administrative burden that is associated with employment protection may make it relatively more costly to fire low-income workers, so that EPL raises the bargaining power of low-income earners more than that of their high-income counterparts (Boeri et al., 2006). The negative effect of EPL on wage dispersion has been found to be stronger in the presence of minimum wages, which prevent firms from passing the cost of EPL on to low-income workers (Koeniger et al., 2007).

- EPL of regular versus temporary contracts: Not only the overall stringency of EPL but also the gap between EPL on regular versus temporary contracts may matter for earnings inequality. If EPL is much stricter for regular than for temporary contracts, this may contribute to higher inequality since workers at the margin of the labour market such as the young risk getting trapped as they move between temporary work and unemployment, with adverse implications for human capital and career progression (OECD, 2004). This premise is tentatively supported by the quantile regressions of Fournier and Koske (2012) that reveal differences between the bottom and the top of the wage distribution. Low-income workers on temporary contracts indeed earn less than those on permanent ones, and this effect fades for workers that are better paid (Figure 7). Moreover, the cross-country panel analysis tentatively indicates that a larger gap between EPL on regular and temporary contracts is associated with more dispersed earnings (Table 1). At the same time more stringent EPL for regular workers appears to be associated with higher unemployment, at least for certain categories of workers (e.g. Bassanini et al., 2010; Murtin et al., 2012).

25. When drawing conclusions from the cross-country time-series regressions for variables other than the minimum wage, the focus is on those specifications that exclude the minimum wage since the inclusion of this variable cuts the sample size approximately in half. 
Figure 6. Union membership - unconditional quantile regression results

Effect on log earnings of raising the share of workers affiliated to a union by one percentage point
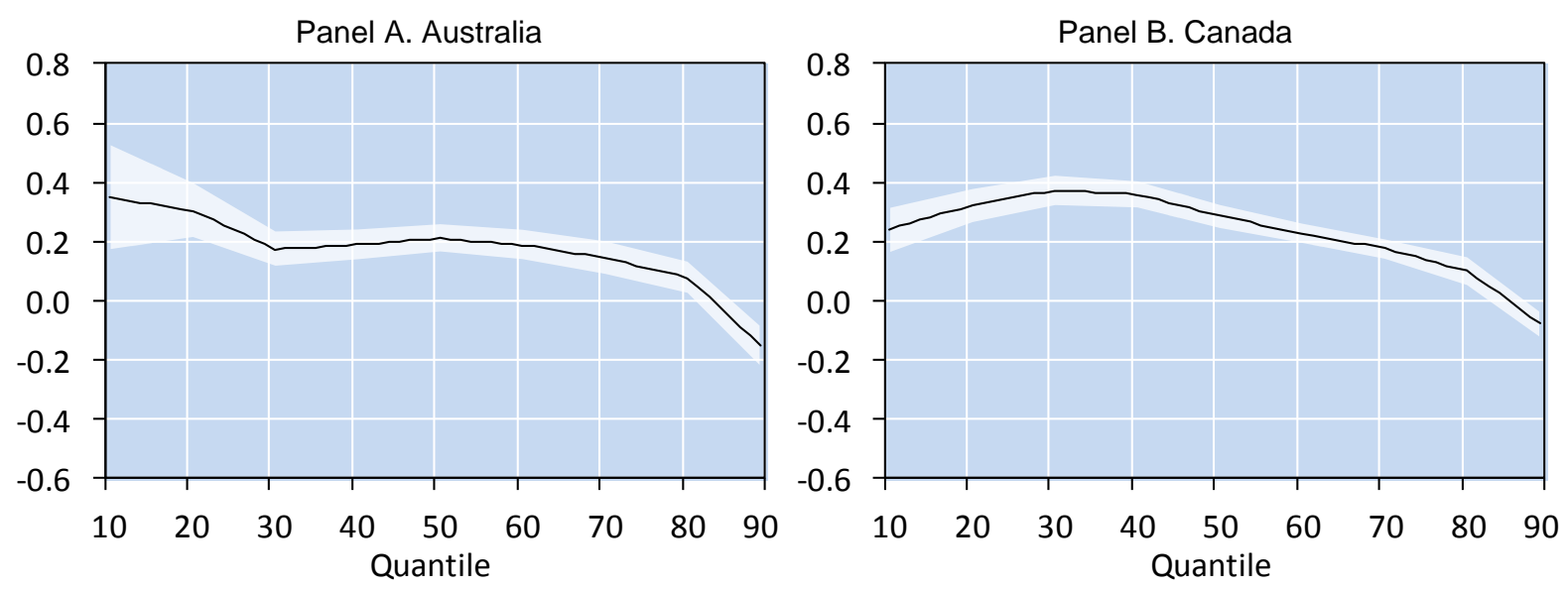

Panel C. Chile

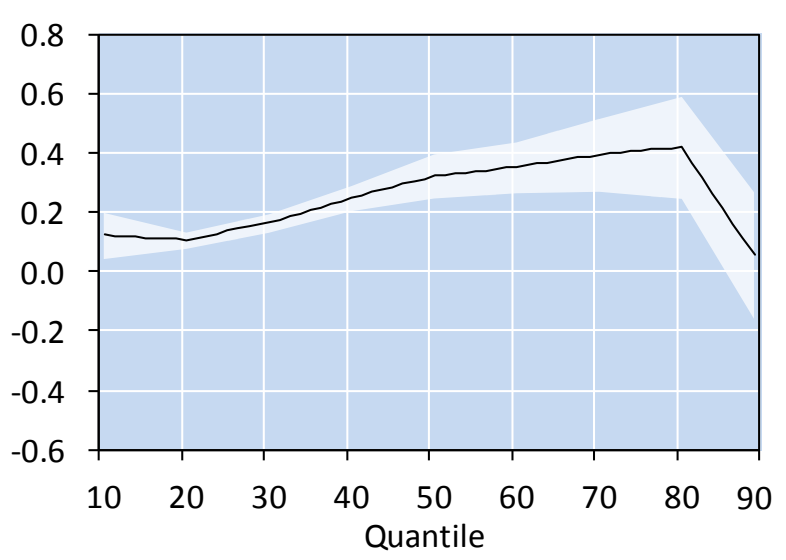

Panel D. Japan

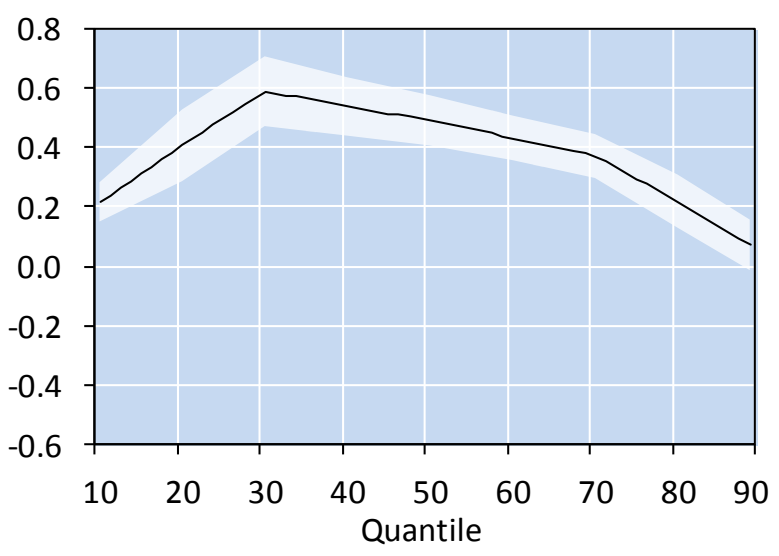

Panel E. Korea

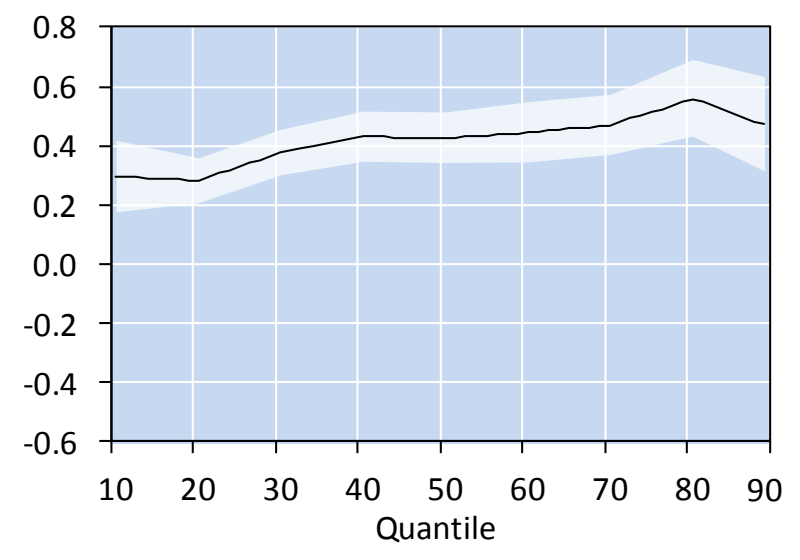

Panel F. Switzerland

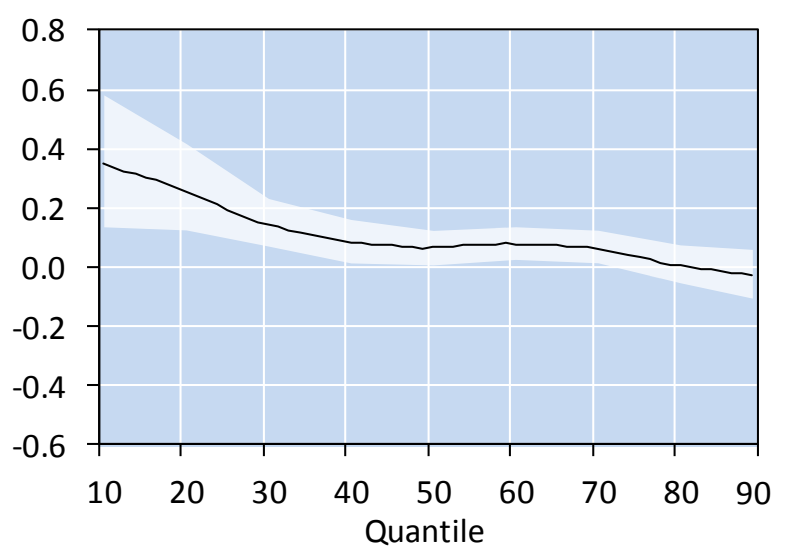


Figure 6. Union membership - unconditional quantile regression results (continued)

Panel G. United States

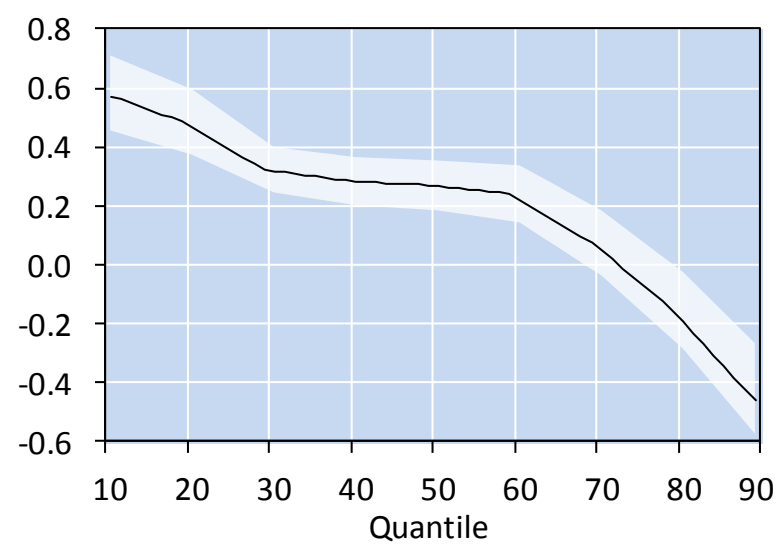

Note: The solid lines show the effect of a 1 percentage point increase in the share of workers affiliated with a union (evenly spread across the population) and the white areas show the $95 \%$ confidence band.

Source: Fournier, J.M. and I. Koske (2012), "Less Income Inequality and More Growth - Are They Compatible? Part 7. The Drivers of Labour Earnings Inequality - An Analysis Based on Conditional and Unconditional Quantile Regressions", OECD Economics Department Working Papers, No. 930.

Figure 7. Earnings effect of having a temporary instead of a permanent work contract

Effect on log earnings of raising the share of workers with a temporary work contract by one percentage point

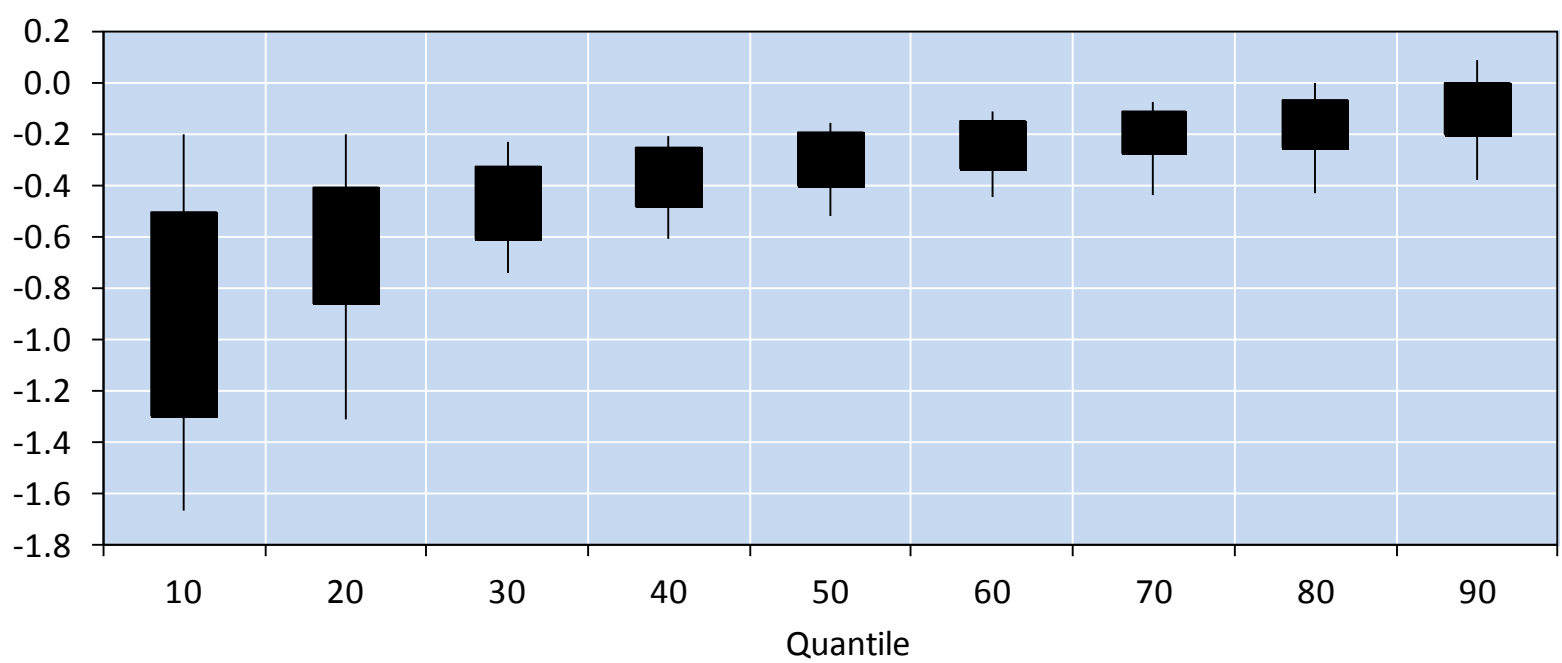

Note: The thick bars depict the cross-country mean of the estimated effect $+/-1$ standard deviation across countries on the logarithm of gross earnings of full-time and part-time workers, while the thin bars depict the crosscountry maximum and minimum of the estimated effect.

Source: Fournier, J.M. and I. Koske (2012), "Less Income Inequality and More Growth - Are They Compatible? Part 7. The Drivers of Labour Earnings Inequality - An Analysis Based on Conditional and Unconditional Quantile Regressions", OECD ECOnomics Department Working Papers, No. 930.

- Unemployment and social assistance benefits: By providing income support, such benefits have a direct income inequality reducing effect (Joumard et al., 2012). At the same time, since unemployment benefits are progressive in most OECD countries due to benefit floors and ceilings that imply relatively higher benefits for people with low income, they may reduce inequality by 
strengthening the bargaining position of low-income workers more than that of high-income workers (Koeniger et al., 2007). However, they may also increase inequality by lowering employment. ${ }^{26}$ The negative employment effects of unemployment benefits are supported both by existing empirical studies (e.g. Nunziata, 2002; Bassanini and Duval, 2006; Murtin et al., 2012; Nickell et al., 2005; OECD, 2011a) and the cross-country time-series regressions carried out in this paper (Table 1). ${ }^{27}$ While Koeniger et al. (2007) and OECD (2011a) find that generous unemployment benefits also compress the wage distribution, the analysis undertaken in this paper does not find support for this view. It appears difficult to establish a significant relationship with broader measures of inequality such as the Gini index (e.g. Vanhoudt, 1997; Burniaux et al. 2006), possibly because the various effects cancel each other.

- Active labour market policies (ALMPs): ALMPs can reduce unemployment and thus labour income inequality through improvements in the job matching process and by raising the skills of the inactive via training programmes. However, the effectiveness of ALMPs in reducing unemployment appears to vary widely across different types of programmes, suggesting that programme design is key (Martin and Grubb, 2001; Kluve and Schmidt, 2002) ${ }^{28}$ In addition, high public spending on ALMPs may contribute to reducing inequality by mitigating the negative employment effects of generous unemployment benefits (Bassanini and Duval, 2006). In line with the positive labour market effects of ALMPs, Vanhoudt (1997) finds that higher spending on ALMPs improves the income share of those at the bottom while reducing the income share of those at the top.

Labour market policies may also shape the way in which globalisation has affected labour income inequality. The cross-country time-series analysis conducted in this study finds, for example, that the effect of trade on labour income inequality may be influenced by institutional features. Trade seems to increase the dispersion of wages among full-time employees if unions are weak, but to have no effect on them if unions are strong (Figure 8, panel A). ${ }^{29}$ By contrast, the negative employment effects of trade are more pronounced under the presence of strong unions (Figure 9), pointing to a role of unions in maintaining existing wage levels in the face of cost-cutting pressure from foreign competition. Moreover, trade is found to increase the dispersion of wages among full-time employees only if employment protection is rather lax, possibly because strict employment protection may delay wage adjustments associated with trade liberalisation (Figure 8, panel B).

26. Specifically, such benefits may reduce the job-search intensity of the unemployed and their willingness to accept job offers (though this effect could be mitigated through more conditionality and activation strategies) and put upward pressure on their wage claims by lowering the economic cost of being unemployed. On the other hand, they may allow jobseekers more time to find better matches, thereby lowering the likelihood of subsequent job separations (Bassanini and Duval, 2006).

27. The conclusions drawn from the cross-country time-series regressions do not change when using the unemployment benefit replacement rate for lower-wage workers instead of the one for all workers.

28. For this reason, no attempt has been made to account for ALMPs in the cross-country time-series regressions presented in Box 1 (adequate data are only available on spending, thus not capturing programme quality and design).

29. Due to the presence of fixed effects in the analysis, no conclusions can be drawn for the long-run equilibrium effects of labour market institutions on the link between trade and inequality. Rather, the estimated effects should be interpreted as referring to the short to medium run. 


\subsection{Product market regulation}

Rents associated with lower product market competition may not be fully appropriated by capital owners but could accrue at least partially to workers. Greater competition, including through the removal of anti-competitive product market regulations, could thus affect earnings inequality through several channels (Nicoletti et al., 2001; Guadalupe, 2007): i) it tends to reduce rents that can be captured by workers through collective bargaining, potentially leading to a decline in union power or more decentralised bargaining, that may, in turn, result in greater labour income inequality; ii) it may induce firms to innovate, which, to the extent that technological advances favour the highly-skilled, could raise wage dispersion; iii) since stringent product market regulations are often associated with wage premia, inter-industry differences in regulation are likely to affect inter-industry wage relativities and thus labour income inequality; $i v$ ) by boosting potential output, product market liberalisation could potentially reduce inequality through higher employment. ${ }^{30}$

There is abundant empirical evidence that product market liberalisation raises employment (e.g. Bassanini and Duval, 2006; Griffith et al., 2007; Nicoletti and Scarpetta, 2005; Fiori et al., 2007; Nicoletti et al., 2001). However, its impact on the dispersion of earnings is less clear-cut: While Nicoletti et al. (2001) could not find much evidence that product market reforms have a long-run impact on earnings inequality, the empirical analysis by Guadalupe (2007) shows that greater competition is associated with a wider earnings distribution. The cross-country time-series evidence by OECD (2011a) also points in this direction, suggesting that product market liberalisation may raise wage dispersion by lowering the relative wages of those at the bottom of the income distribution. However, the cross-country time-series regressions conducted in this paper suggest that this result is not robust across different specifications (Table 1).

Figure 8. The interaction between labour market institutions and trade - wage dispersion

Percentage point change in $90 / 10$ percentile of full-time employees ratio due to a $1 \%$ increase in the trade-to-GDP ratio as a function of:

Panel A. Union density

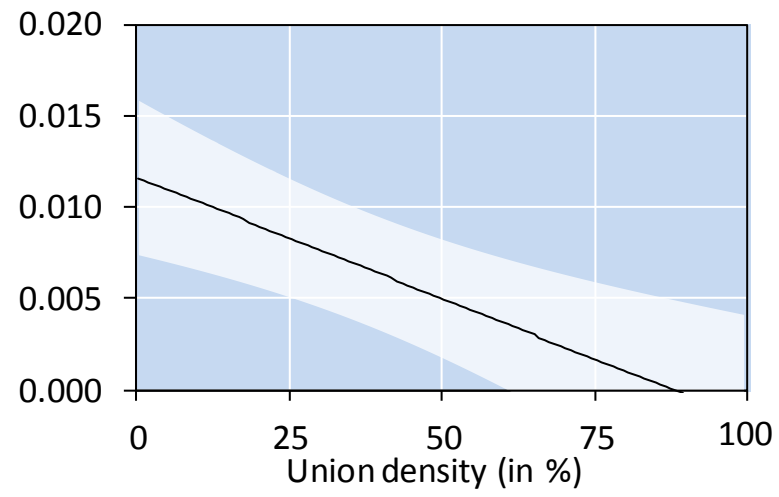

Panel B. Employment protection legislation

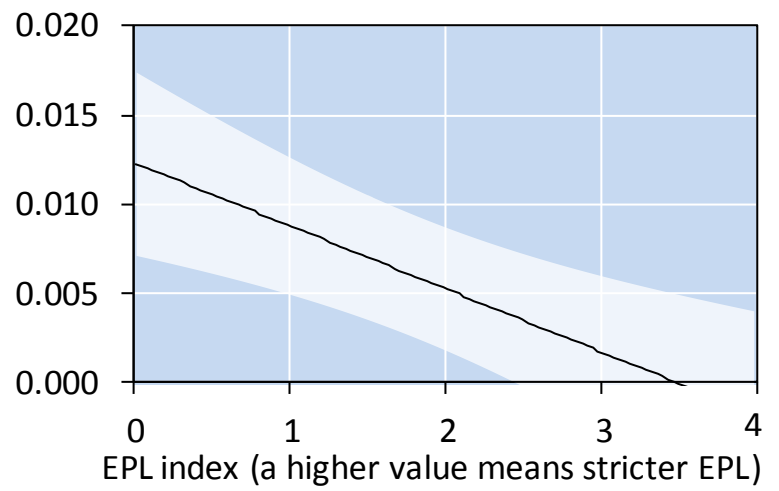

Note: The lines show the point estimates of the effect of trade on the 90/10 decile ratio among full-time employees and the shaded areas indicate the $90 \%$ confidence intervals.

Source: Based on the coefficients estimates presented in Table 3.

30. Product market regulation can affect income inequality also through its impact on relative prices. For example, relative price declines resulting from regulatory reform in a particular industry will advantage those groups who are intensive consumers of this industry's products (Nicoletti et al., 2001). 
Figure 9. The interaction between labour market institutions and trade - employment

Percentage point change in the employment rate due to a $1 \%$ increase in the trade-to-GDP ratio as a function of union density

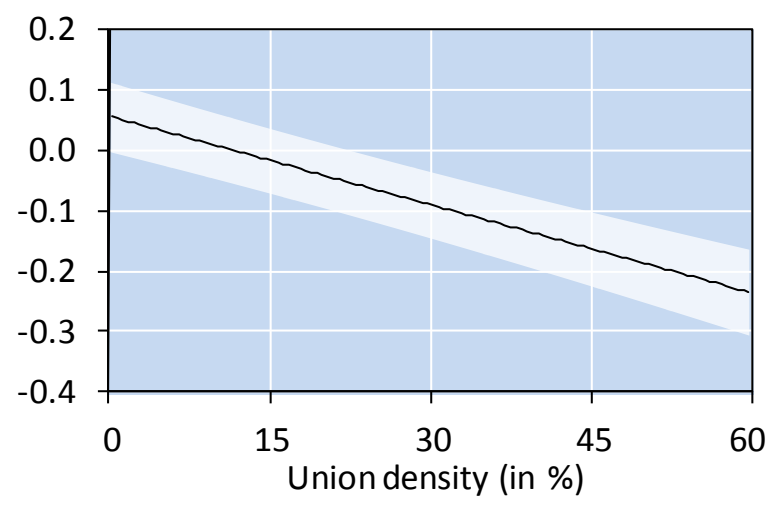

Note: The line shows the point estimate of the effect of trade on the $90 / 10$ decile ratio among full-time employees and the shaded area indicates the $90 \%$ confidence interval.

Source: Based on the coefficients estimates presented in Table 3.

\subsection{Tax policy}

The tax system influences inequality by altering the after-tax distribution of income. While this effect is discussed in detail in Joumard et al. (2012), tax policy may also influence the distribution of pre-tax earnings through its impact on labour demand and supply. In a perfectly competitive labour market higher labour taxes should not affect equilibrium unemployment since workers will bear the entire tax burden through lower net wages. However, if firms cannot shift the entire tax burden onto workers (for example, because of minimum wages or strong trade unions) higher taxes will reduce labour demand. Several empirical studies support this view (e.g. Belot and van Ours, 2004; Bassanini and Duval, 2006; Murtin et al., 2012). The impact appears to be particularly strong in countries with strong trade unions and an intermediate (or low) degree of wage bargaining coordination (e.g. Elmeskov et al., 1998; Daveri and Tabellini, 2000; Murtin et al., 2012). To the extent that job losses are more prevalent among low-wage workers, a higher tax wedge may, however, reduce the dispersion of wages (among those who retain their job). New OECD evidence tentatively supports this premise (OECD, 2011 $a$ and Table 1).

\subsection{Other policy issues}

\section{Gender inequality}

Inequality among women and among men has increased between the mid-1990s and mid-2000s in the majority of OECD countries (Figure 10). In many countries, the increase in inequality was more pronounced among men than among women. At the same time, despite some convergence between men and women, gender differences in labour market performance are still striking in most countries. Women are less likely to be employed than men (on average across countries, $76 \%$ of men of working age are in employment compared with $67 \%$ of women) and those who are working typically earn less than their male counterparts (on average across countries, women earn one-third less than men). Using an Oaxaca-Blinder-type decomposition to break down the gender earnings gap into differences in personal characteristics and differences in the returns to these characteristics suggests the following (Figure 11):

- Women's shorter working hours (which are most likely due to the fact that they take on more caring obligations for children and elderly relatives than men; see OECD 2011b) play an important role in 
explaining differences in earnings between men and women - a finding that is in line with previous evidence (e.g. Ponthieux and Meurs, 2005).

- Secondary and tertiary attainment rates of women are equal to or exceed those of men in most OECD countries as reflected in a zero or negative contribution of the composition of education to the gender earnings gap. ${ }^{31}$

- In the majority of countries, gender differences in the choice of occupation and/or sector of employment raise the gender wage gap - notable exceptions are Ireland, Poland and Portugal.

- A large part of the gender earnings gap is explained by the constant and rate-of-return effects (i.e. differences between men and women in the returns to personal characteristics such as age or the level of education). While this might be due to the omission of important variables in the regression, ${ }^{32}$ it may also capture gender discrimination.

Figure 10. Annual change in inequality among women and men between the mid-1990s and the mid-2000s

Difference in logarithm of percentile ratios (full-time employees)

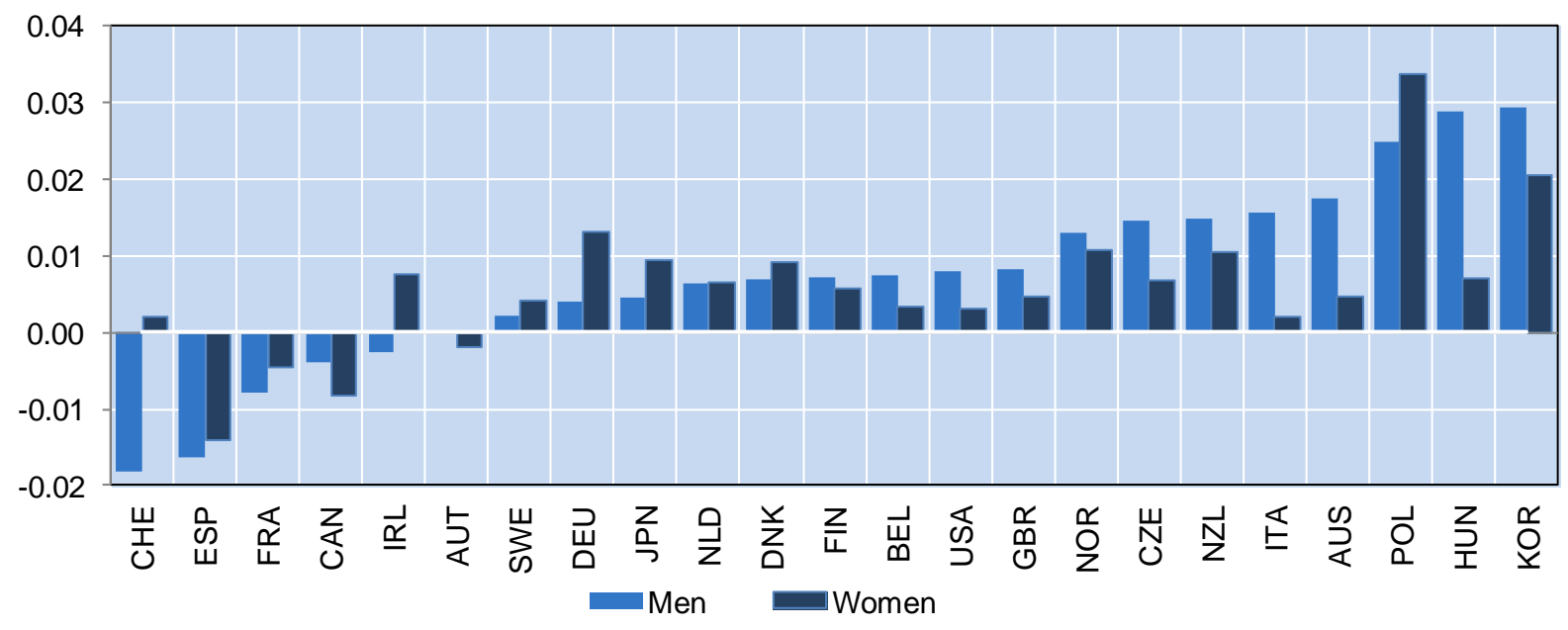

Note: $\quad$ The mid-1990s refer to 1995 for all countries but Canada (1994), Ireland (1997), Denmark (1996), Norway (1997), Belgium (1990) and the Czech Republic (1996). The mid-2000s refer to 1995 for all countries but Poland (2004).

Source: National household and enterprise surveys.

31. Flabbi (2011) shows that although women are slightly more likely than men to obtain a tertiary degree, these are more often in the first level of tertiary education (e.g. Bachelor) than in the post-graduate level (e.g. Masters or Ph.D.).

32. For example, women might lack behind men in job market experience caused by career breaks around childbirth (e.g. Dupuy and Fernández-Kranz, 2011; Buligescu et al., 2008) or might pursue different fields of study which may give rise to different returns to tertiary education. 
Figure 11. Decomposition of the gender earnings gap

Full-time and part-time workers, 2008 or latest available year ${ }^{1}$

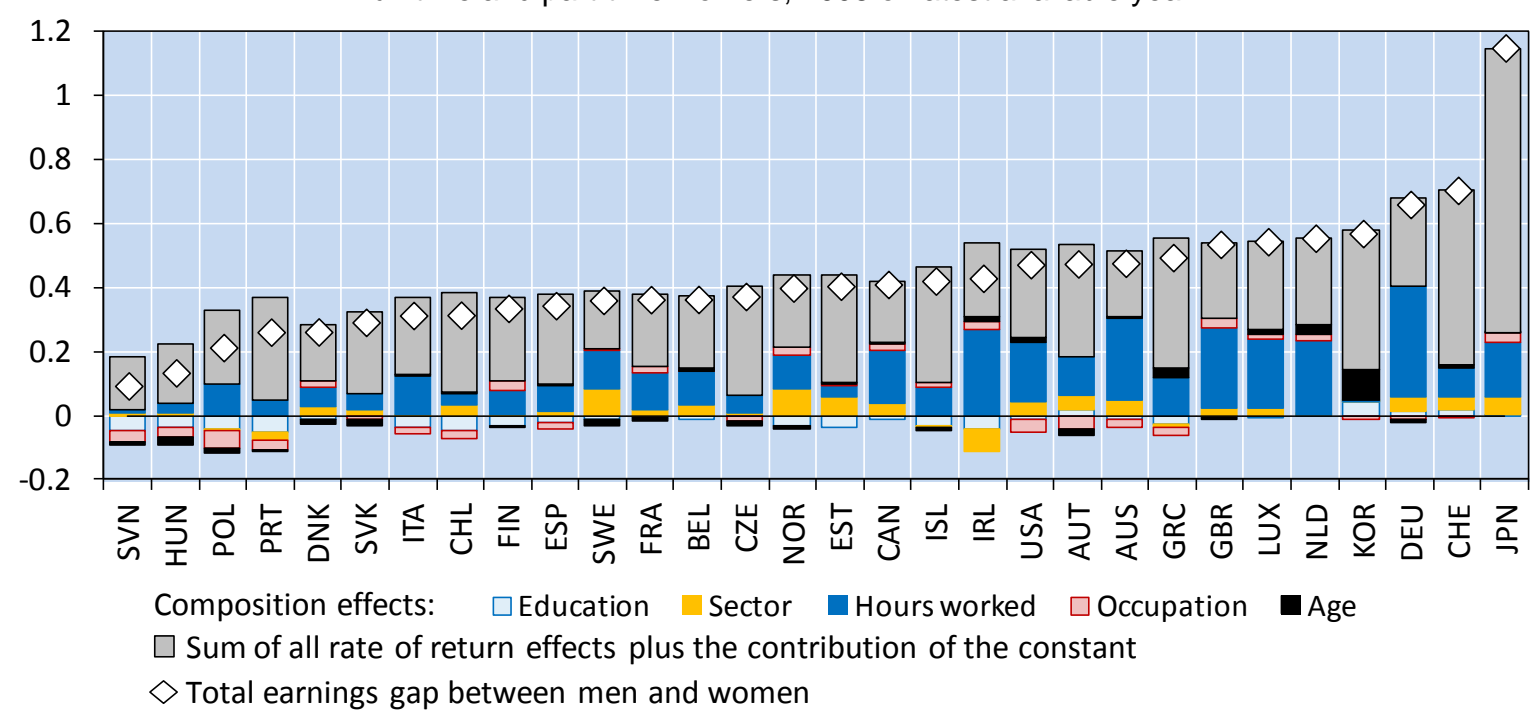

1. 2009 for Australia and Japan; 2007 for France, Korea and the United States. Ratio between men's and women's average log

earnings.

Source: Panel Study of Income Dynamics (PSID) for the United States; Household Income and Labour Dynamics in Australia Survey (HILDA) for Australia; Survey of Labour and Income Dynamics (SLID) for Canada; National Socioeconomic Characterization Survey (CASEN) for Chile; Korean Labour and Income Panel Study (KLIPS) for Korea; Japan Household Panel Survey (JHPS) for Japan; Swiss Household Panel (SHP) for Switzerland; European Union Statistics on Income and Living Conditions (EU-SILC) for the other countries.

\section{Migration}

Migration influences labour earnings inequality in the host country because $i$ ) the labour market outcomes of foreign-born differ from those of natives and ii) immigrants alter the labour market outcomes of natives. ${ }^{33}$ As regards the former effect, immigrants tend to under-perform natives in terms of employment, earnings or both, suggesting that immigration raises inequality through this channel. ${ }^{34}$ Decomposing the average earnings gap between natives and immigrants into differences in personal characteristics and differences in the returns to these characteristics points to considerable cross-country heterogeneity (Figure 12), which may, for example, be due to differences in the structure of the immigrant population (in terms of country of origin, timing of immigration waves or motivation) or differences in countries' policy settings. Two factors that contribute positively to the gap in many countries are a lower average level of education of immigrants and the sector composition, with immigrants working in sectors that pay less well. In addition, the contribution of the constant and rate-of-return effects raise the gap, as migrants earn less than natives, even if they have exactly the same characteristics (in terms of age, gender, education level and so on). This may, for example, reflect firms' difficulties in properly assessing qualifications obtained in a foreign country, the fact that at least some of the immigrants may have acquired their skills in a lower-quality educational environment, immigrants' lack of work experience in

33. At the same time, the policy setting and the level of earnings inequality in the destination country (relative to that in the source country) may influence migration flows (see Liebig and Sousa-Poza, 2004, for a brief overview of the theoretical underpinnings as well as empirical evidence).

34. Even though the gap decreases over time as immigrants assimilate, it typically persists even after a long period of stay (Jean et al., 2010). 
the host country (which is an important channel of integration, but not controlled for in the regression), and also discrimination.

Figure 12. Decomposition of the earnings gap between natives and immigrants

Full-time and part-time workers, 2003 to latest available year

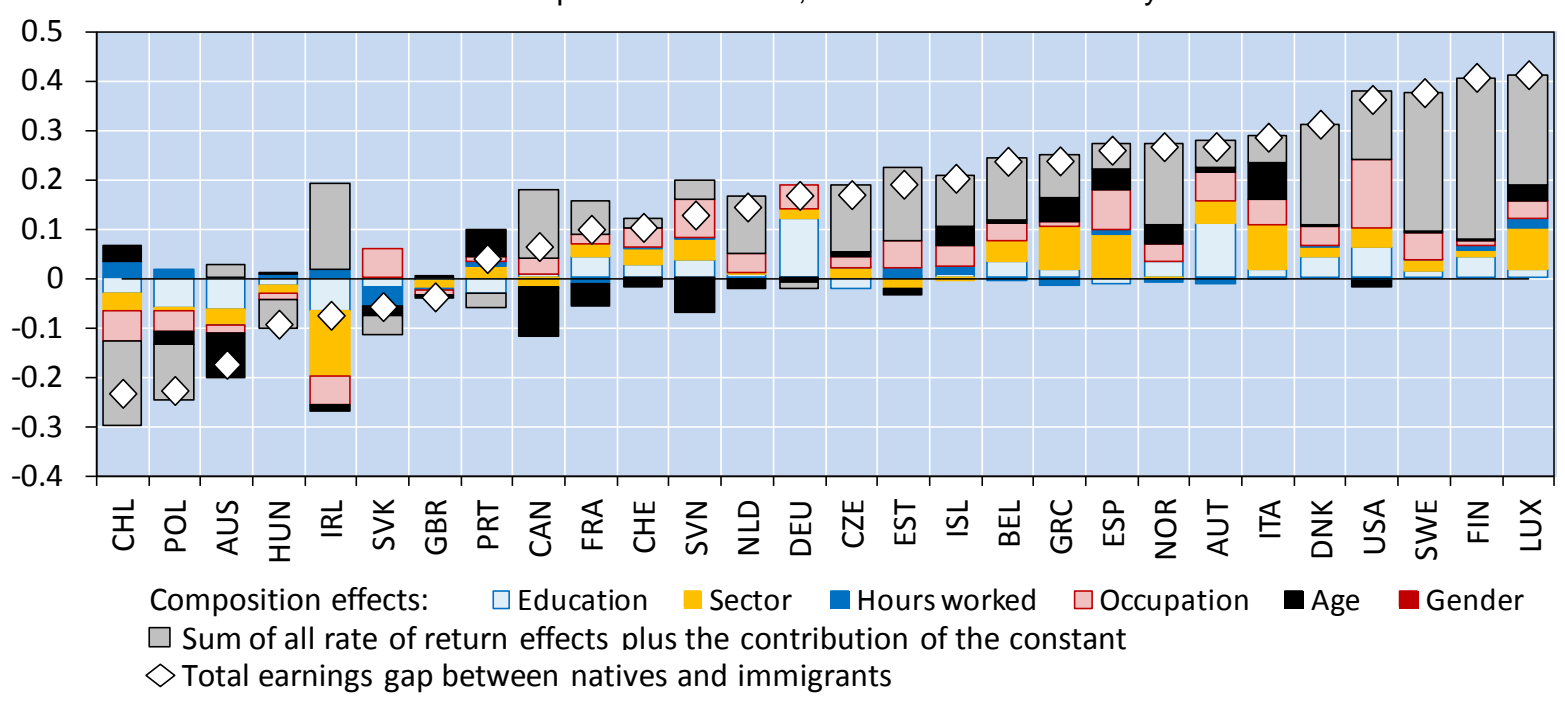

Note: Individuals are defined as immigrants if they are born outside the country or, in case of EU member countries, outside the EU. The earnings gap is defined as the ratio between natives' and immigrants' average log earnings.

Source: Panel Study of Income Dynamics (PSID) for the United States; Household Income and Labour Dynamics in Australia Survey (HILDA) for Australia; National Socioeconomic Characterization Survey for Chile; Korean Labour and Income Panel Study (KLIPS) for Korea; Luxembourg Income Study (LIS) for Israel; Japan Household Panel Survey (JHPS) for Japan; Swiss Household Panel (SHP) for Switzerland; European Union Statistics on Income and Living Conditions (EU-SILC) for the other countries.

While there is not much evidence that immigrants have a sizable impact on the employment rate of natives with similar skills or occupations (e.g. Bonin, 2005; Cohen-Goldner and Paserman, 2004; Jean and Jiménez, 2011; D'Amuri and Peri, 2011), they appear to reduce the wages of workers with similar skills or occupations (e.g. Borjas, 2003; Aydemir and Borjas 2007; Orrenius and Zavodny, 2007; Steinhardt, 2011). At the same time, the wages of natives whose skills are complementary to those of immigrants (Ottaviano and Peri, 2012, point to sizable complementarities in the case of the US) may rise in response to the arrival of new immigrants. The impact of immigration on earnings inequality among natives is thus unclear a priori and depends in particular on the skill mix of the immigrant population. Aydemir and Borjas (2007) who investigate the impact of immigration in countries with very different skill-structures among immigrants find that it reduces inequality in Canada (characterised by high-skilled immigration), but increases it in the United States (low-skilled immigration). In many OECD countries the distribution of educational attainment among immigrants is increasingly U-shaped, with an over-representation of both highly skilled and unskilled individuals (Jean et al., 2010). This suggests that the inequality effects of immigration are likely to be small in many countries, with the effect on highly and less educated natives offsetting each other at least to some extent.

Migration may influence earnings inequality also in the source country. Most importantly, emigration alters the skill-composition of the workforce in the source country and, thus, the relative returns to skills. The direction of the impact on earnings inequality is theoretically ambiguous and depends in particular on the skill mix of emigrants and the substitutability between low-skilled workers, high-skilled workers and capital (Davies and Wooton, 1992). Aydemiar and Borjas (2007) find for Mexico, for example, that 
emigration has increased relative wages in the middle of the skill distribution (where emigration rates are highest in the case of Mexico) and lowered the relative wages at the extremes.

\section{Identifying reform options to reduce labour income inequality}

As discussed in the previous section, structural policies influence labour income inequality through their impact on employment and the dispersion of earnings. To identify reform options for individual OECD countries that could help them to reduce inequality, country diamonds have been constructed which show for each country the link between various policies and earnings equality as well as employment. In addition, the diamonds show countries' performance with respect to equality of opportunity. For each policy indicator a value above the OECD average means that the country is better positioned than the OECD average to reduce inequality and vice versa.

Figure 13 illustrates the use of these diamonds using the example of Canada (see the Annex for other OECD countries). The indicator set shows that in Canada labour and product market regulations tend to be more employment-friendly than the OECD average (light blue shaded area), but they have adverse effects on the equality of earnings (dark blue shaded area). The education system delivers more equitable outcomes than the systems of most other OECD countries, as indicated by a smaller impact of pupils' socioeconomic background and area of residence (rural versus urban) on PISA scores (white shaded area). This is also reflected in relatively high inter-generational social mobility: the impact of parental earnings on the earnings of their offspring is relatively small. However, intra-generational mobility is relatively low: both the probability of remaining poor once fallen into poverty (indicator on poverty mobility) and the probability of staying in a given income quintile (indicator on income mobility) are high compared with other OECD countries. This does not seem to be driven by difficulties in re-entering the labour market after a spell of unemployment, as the share of long-term unemployed (defined here as those who are unemployed for more than one year) is somewhat lower than in other countries.

\section{Figure 13. Indicators of policies that influence labour income inequality - the example of Canada}

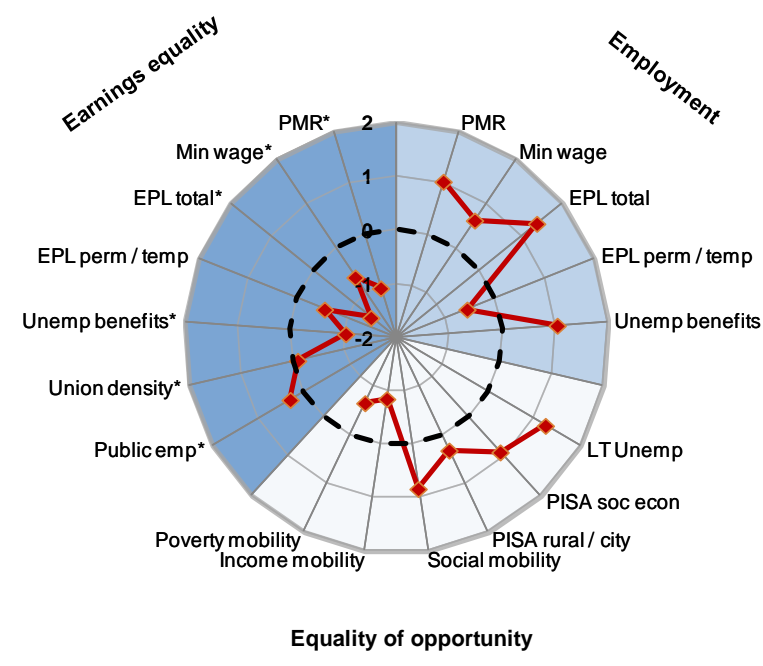
PMR = Economy-wide product market regulation
Min wage = Ratio of minimum to median wage
Union density $=$ Share of workers affiliated to a trade union
$\mathrm{EPL}=$ Employment protection legislation
EPL perm/temp = Difference in EPL between permanent and temporary workers
Unemp benefits $=$ Average gross unemployment benefit replacement rate
Public emp $=$ Employment in general government and public corporations as $\%$ of total labour force
LT Unemp $=$ Long-term unemployed as share of total unemployed
PISA soc econ $=$ Average reading score point difference associated with socio-economic background
$\mathrm{PISA}$ rural/city = Reading performance, difference between students attending city schools vs. rural/village schools
Social mobility = Intergenerational earnings elasticity
Income mobility = Average share of people staying within $\mathrm{HDI}$ quintile (average of all quintiles)
Poverty mobility = Share of people experiencing persistent poverty

Note: The dotted line represents the OECD average, the solid line and diamond markers represent the country shown. Where the solid line falls outside the OECD average, this implies better results than the OECD average with regard to the three policy goals shown. The series with asterisks have been inversed to take into account the relationship between these policy variables and the policy goal. The indicators are presented in standard deviation units. 


\section{Reducing labour income inequality and boosting GDP per capita: policy trade-offs and complementarities}

The primary goal of structural reforms is to improve long-run living standards. As part of its Going for Growth process, the OECD analyses the link between structural policies and GDP per capita and translates the results into concrete policy recommendations. At the same time, as illustrated above, most of the reforms recommended in Going for Growth also influence income inequality, which is another policy objective (see Table 4 for a summary of the empirical findings obtained in the present as well as in previous studies). Against this background, this section uses the results from this study and previous OECD work on the drivers of growth - most of which has been published in various editions of Going for Growth - to assess possible policy trade-offs and complementarities between growth and income distribution objectives. In doing so, growth-enhancing policy reforms are classified into three categories: i) those that are likely to reduce income inequality; ii) those that are likely to raise it; and iii) those that seem to have an ambiguous effect. While the discussion focuses on labour income inequality, any fall in labour income inequality should translate into a fall in disposable income inequality, all else equal (in particular the tax and transfer system).

Table 4. The impact of structural policies on labour income equality

\begin{tabular}{|c|c|c|c|c|}
\hline A rise in: & $\begin{array}{l}\text { Employment } \\
\text { rate }\end{array}$ & $\begin{array}{l}\text { Earnings } \\
\text { equality }\end{array}$ & $\begin{array}{l}\text { Total labour } \\
\text { income } \\
\text { equality }\end{array}$ & $\begin{array}{c}\text { GDP per } \\
\text { capita }\end{array}$ \\
\hline Initiatives to increase the tertiary graduation rate & $\sim$ & + & $(+)$ & + \\
\hline Initiatives to increase the upper-secondary graduation rate & $\sim$ & + & $(+)$ & + \\
\hline Initiatives to promote equity in education & $\sim$ & + & $(+)$ & + \\
\hline The minimum wage (as share of the median wage) & $0 /-$ & + & $\sim$ & $(0 /-)$ \\
\hline Unionisation & $\sim$ & + & + & $(\sim)$ \\
\hline Legal extensions of collective wage agreements & - & $\sim$ & $(-)$ & $(-)$ \\
\hline The overall level of EPL & $0 /-$ & + & $\sim$ & - \\
\hline The gap between EPL on regular versus temporary work & - & - & $(-)$ & - \\
\hline Replacement rate and duration of unemployment benefits & - & + & $\sim$ & - \\
\hline Spending on active labour market policies & $0 /+$ & $\sim$ & + & + \\
\hline Anti-competitive product market regulation & - & $0 /+$ & $\sim$ & - \\
\hline Initiatives to foster the integration of immigrants & + & + & $(+)$ & $(+)$ \\
\hline Initiatives to combat discrimination in the labour market & + & + & $(+)$ & $(+)$ \\
\hline Initiatives to raise female labour force participation & + & + & $(+)$ & $(+)$ \\
\hline Initiatives to avoid gender stereotyping in education & $\sim$ & + & $(+)$ & $(\sim)$ \\
\hline
\end{tabular}

Note: The term "earnings inequality" refers to inequality among the working population and the term "total labour income inequality" refers to inequality among the working-age population, thus accounting for both employment and earnings inequality effects.

,,+- 0 denote respectively a significant rise, a significant fall, and no impact on the variable of interest. In cases where some studies find a significant effect while other don't, this is indicated by combining the symbols; e.g. 0/+ means that some studies cannot find a significant effect while others find a positive effect or that studies find a significantly positive effect only on some groups of workers but not on all of them. A tilde means that the sign of the effect is unknown because the empirical literature is inconclusive or because studies on the link are not available. When the sign of the total labour income inequality effect is unknown but can be deducted from the signs of the employment and earnings equality effects, the results are reported in brackets. The GDP per capita effects are based on the findings of previous OECD and other studies (reported e.g. in Barnes et al., 2011 and Bouis and Duval, 2011) or deducted from the employment rate effect (in which case the GDP per capita effect is written in brackets).

Caution is needed in interpreting the results for a number of reasons. First, the results may not reflect the overall (general equilibrium) effects of policy changes. This may, for example, be the case whenever the conclusions are based on the quantile regression framework by Fournier and Koske (2012). Moreover, the question of how policy reforms are financed is ignored, meaning that the effects shown here are only partial. ${ }^{35}$ Second, the uncertainties that surround the estimated effects are not taken into account. Third, no

35. See Joumard et al. (2012) for a discussion of the trade-offs and complementarities of tax policy reform with respect to the growth and income distribution objectives. 
comparison is made of the magnitudes of the effects on GDP per capita and income inequality. Fourth, the focus is on labour income inequality instead of overall income inequality, meaning that important population subgroups (such as retirees) and income sources (such as capital income) are ignored. The reason for this restricted focus is the lack of studies on the linkages between many of the policies considered and overall income inequality. Fifth, it abstracts from possible additional direct interactions between economic growth and inequality. The reason is that although these interactions have attracted great attention in the economic debate in recent years, both the theoretical and the empirical literature remain by and large inconclusive regarding both causality and the direction of influence (see Fields, 2001 and Ehrhart, 2009 for surveys of the vast literature).

\section{Growth enhancing policy reforms that are likely to reduce income inequality}

- Improving the quality and quantity of education: Reforms that facilitate the accumulation of human capital are among the most important for improving long-run living standards (OECD, 2011c). Examples of reforms include inter alia enhanced accountability and autonomy for both secondary and tertiary education institutions and better teacher recruitment and training. Although it can take up to a generation until all the GDP per capita gains from such reforms have been realised, small improvements in the skills of a nation's labour force can have a large effect on future GDP per capita (OECD, 2010b). Many of the reforms are likely to have additional benefits in terms of a more equitable distribution of labour income and at least some of them could be achieved without raising government spending. Since an upper secondary degree is today generally a prerequisite for being successful in the labour market in most OECD countries, reducing the number of pupils that leave school without a lower-secondary degree (e.g. by providing support to pupils at risk to reduce drop outs) should help reduce income inequality. Similar benefits can be expected from reforms that encourage more students to pursue tertiary studies. Although a rise in the number of tertiary graduates raises inequality via a pure composition effect according to the quantile regressions (at least until highly-educated workers reach a certain share of the workforce), there is some tentative evidence from the cross-country time-series regressions that this effect is more than offset by a decline in the relative returns to tertiary education enough to more than offset this effect. ${ }^{36}$ Still, tuition fees that make students share at least part of the cost of tertiary education could lower inequality as the current financing of education is regressive, provided the poor are not excluded from tertiary education - for example by combining tuition fees with student loans whose repayment is contingent on income. ${ }^{37}$

- Promoting equity in education: Making educational outcomes less dependent on personal and social circumstances should boost GDP per capita by enhancing entrepreneurship, work incentives, the overall quality and allocation of human capital and, ultimately, productivity (OECD, 2011b). At the same time, a more equitable distribution of educational opportunities has been shown to entail a more equitable distribution of labour income. Examples of policy initiatives that have been shown to raise equity in education include postponing early tracking (e.g. Hanushek and Wößmann, 2006; Bauer and Riphahn, 2005) and strengthening links between school and home to help disadvantaged children to learn (e.g. Mullis et al., 2003). Providing early childhood care and basic schooling for all is also key as it may yield large positive returns over the entire lifetime, particularly for the most disadvantaged (Chetty et al., 2011; McCabe and Smyth, 2000; Leseman, 2002; OECD, 2006a).

36. While a high share of workers with a $\mathrm{PhD}$ degree should be beneficial for innovation and growth, it may raise the share of top-income earners, thus contributing to higher income inequality.

37. Empirical evidence suggests that any negative effect of tuition fees on participation rates can be fully offset through improvements in the financial support for students (Santiago et al., 2008; Heller, 1999). Looking at household data from the province of Québec in Canada, Makdissi and Yazbeck (2011) also conclude that levying low higher-education tuition fees is not regressive. 
Similarly, carefully managing school choice to prevent that pupils with weaker parental support are at a disadvantage, and channelling resources to students and regions with the greatest needs, removing dead ends in upper secondary education and offering a second chance to those who fail at school through, for example, the recognition of informal learning may help to achieve more equity in education (see OECD, 2007 for detailed policy recommendations on how to promote equity in education).

- Reducing the gap between employment protection on temporary and permanent work: A smaller gap in job protection between temporary and permanent contracts lowers income inequality by improving the labour market prospects of those at the margin of the labour market (e.g. the youth) both in terms of employment and wages. It is also likely to reduce the income gap between immigrants and natives, as immigrants also suffer disproportionately from labour market dualism (Causa and Jean, 2007). The associated human capital accumulation may spur productivity improvements which, in turn, lead to higher GDP per capita.

- Increasing spending on active labour market policies: As ALMPs are often accompanied by a strong emphasis on activation, they may limit the adverse effects of high social benefits on work incentives and employment, thereby contributing to higher GDP per capita and, abstracting from their financing, lower labour income inequality. The same effects may arise from ALMP-driven improvements in skills and job matching - to the extent that they benefit disproportionately marginal labour market groups.

- Promoting the integration of immigrants: Targeted policies such as language courses and transparent systems of recognising foreign qualifications should help reduce labour income inequality by closing the gap between immigrants and natives' labour market performance. ${ }^{38}$ Better labour market integration of immigrants should also raise GDP per capita as it raises labour force participation and improves the allocation of human capital (and, thereby, productivity).

- Fostering female labour market participation: Since women tend to take on more caring responsibilities than men, they work fewer hours and thus take home less pay. Ideally, the higher labour supply elasticity of women should be dealt with by taxing them at a lower rate than men. Since this is not feasible in practice, policies to improve the availability of formal care for children and the elderly can serve as a second best solution. Such policies should help to reduce gender differences in working hours and pay, and at the same time improve long-run living standards through higher participation rates. Such policies might further contribute to higher earnings equality by influencing women's career choices. ${ }^{39}$

- Avoiding gender stereotyping in education: To the extent that differences in occupational choice do not reflect only personal preferences but also gender stereotyping they need to be addressed. This should reduce inequality between men and women with a positive effect on GDP per capita through better allocation of resources.

- Fighting discrimination: The analyses presented above suggest that at least part of the earnings gap between men and women and between immigrants and natives is due to discrimination.

38. Such policies may also influence the size of migration inflows as immigration is endogenous to the policy setting in the country.

39. As women traditionally take career breaks for child rearing reasons, they may choose occupations with flatter earnings profiles (Polachek, 1981), or trade lower earnings for job characteristics that improve their family life such as shorter commuting or flexible hours (Killingsworth, 1987). 
Discrimination is likely to entail negative consequences for long-run living standards as it reduces work incentives and leads to a suboptimal allocation of human capital. ${ }^{40}$ To combat discrimination, legal rules can be made more effective, for example by empowering well-resourced specialised bodies to investigate employers even in the absence of individual complaints and to take legal action against those who engage in discriminatory practices (OECD 2011b).

\section{Growth enhancing policy reforms that are likely to raise income inequality}

- Increasing the flexibility of wage determination: Administrative extensions of collective wage agreements to firms that are not party to the original settlement may set labour costs at too-high levels for some employers, thus hampering productivity through lower competitive pressures from the entry of new firms and possibly reducing employment. At the same time, the less dispersed wages among union members indicate that such extensions may be associated with lower earnings inequality. While empirical evidence also suggests that centralised as opposed to intermediate wage bargaining might be beneficial for both employment and income equality, the level of bargaining is not under the immediate influence of policy makers.

\section{Growth enhancing policy reforms that have an uncertain impact on income inequality}

- Avoiding too high and long-lasting unemployment benefits: If unemployment benefits are too high or long-lasting, they risk reducing job-search incentives and raising wages above market clearing levels, thereby lowering employment with negative effects on GDP per capita (OECD, 2006b). These labour market effects have implications for income inequality: the lower employment rate is likely to be associated with higher income inequality, whereas the more compressed wage distribution - to the extent that unemployment benefits are progressive or lower-income workers are more likely to receive them - has the opposite effect. While in the short run, these effects on inequality are likely to be dominated by the direct inequality-reducing effect that stems from the income support for the unemployed (see Joumard et al., 2012), the direction of the overall effect is less clear-cut in the long run. However, cutting back on benefits may help to reduce the income gap between immigrants and natives as the former appear to suffer more from adverse employment effects (Causa and Jean, 2007).

- Liberalising product markets: A wide range of industry and country-level studies illustrate the large beneficial effects of product market liberalisation on the pace of productivity convergence to technologically advanced countries (e.g. Bourlès et al., 2010, Conway et al., 2006). At the same time, the impact of product market liberalisation on labour income inequality is uncertain: while higher employment should reduce inequality, ceteris paribus, this may potentially be offset by a wider earnings dispersion.

- Lowering minimum labour costs: If minimum wages are set too high, they risk limiting the job market opportunities of young workers and the low skilled. Under such circumstances, lowering relative labour costs may boost employment of marginal groups in the labour market. Higher employment raises GDP per capita and reduces labour income inequality, but a wider wage distribution raises it, ceteris paribus, resulting in an ambiguous effect. The employment effect is likely to be the smaller the lower the initial level of minimum labour costs, in which case the likelihood that cuts result in higher labour income inequality will be greater.

40. Livanos et al. (2009) provide some evidence that discrimination contributes to lower employment rates of women relative to men's. 


\section{Bibliography}

Abiad, A., E. Detragiache and T. Tressel (2010), “A New Database of Financial Reforms”, IMF Staff Papers, Vol. 57, pp. 281-302.

Acemoglu, D. (2002), “Technical Change, Inequality, and the Labor Market”, Journal of Economic Literature, Vol. 40, No. 1, pp. 7-72.

Acemoglu, D. and D.H. Autor (2010), "Skills, Tasks and Technologies: Implications for Employment and Earnings”, NBER Working Papers, No. 16082, National Bureau of Economic Research.

Amiti, M. and D.R. Davis (2008), "Trade, Firms, and Wages: Theory and Evidence", NBER Working Papers, No. 14106, National Bureau of Economic Research.

Arulampalam, W. and A.L. Booth (2001), "Learning and Earning: Do Multiple Training Events Pay? A Decade of Evidence from a Cohort of Young British Men", Economica, Vol. 68, No. 271, pp. 379-400.

Autor, D.H., F. Levy and R. Mumane (2003), “The Skill Content of Recent Technological Change: An Empirical Exploration”, Quarterly Journal of Economics, Vol. 118, No. 4, pp. 1279-1333.

Autor, D.H., L.F. Katz and M.S. Kearney (2006), "The Polarization of the US Labour Market", American Economic Review, Vol. 96, No. 2, pp. 189-194.

Aydemir, A. and G.J. Borjas (2007), "Cross-Country Variation in the Impact of International Migration: Canada, Mexico, and the United States", Journal of the European Economic Association, Vol. 5, No. 4, pp. 663-708.

Barnes, S. et al. (2011), "The GDP Impact of Structural Reform: A Simple Simulation Framework", OECD Economics Department Working Papers, No. 834.

Bassanini, A. et al. (2005), "Workplace Training in Europe”, IZA Discussion Papers, No. 1640.

Bassanini, A. and R. Duval (2006), "Employment Patterns in OECD Countries: Reassessing the Role of Policies and Institutions", OECD Economics Department Working Papers, No. 486.

Bassanini, A., A. Garnero, P. Marianna and S. Martin (2010), "Institutional Determinants of Worker Flows: A Cross-Country/Cross-Industry Approach", OECD Social, Employment and Migration Working Papers, No. 107.

Bauer, P. and R. Riphahn (2005), "Timing of School Tracking as a Determinant of Intergenerational Transmission of Education”, Economics Letters, Vol. 91, pp. 90-97.

Belot, M. and J. Van Ours (2004), "Does the Recent Success of Some OECD Countries in Lowering their Unemployment Rates Lie in the Clever Design of their Labour Market Reform?", Oxford Economic Papers, Vol. 56, No. 4. 
Boeri, T., J. Ignacio Conde-Ruiz and V. Galasso (2006), “The Political Economy of Flexicurity”, mimeo, Bocconi University.

Bonin, H. (2005), "Wage and Employment Effects of Immigration to Germany: Evidence from a Skill Group Approach", IZA Discussion Papers, No. 1875.

Boone, J. (2000), “Competition”, CEPR Discussion Papers, No. 2636.

Borjas, G.J. (2003), "The Labor Demand Curve Is Downward Sloping: Reexamining the Impact of Immigration on the Labor Market", Quarterly Journal of Economics, Vol. 118, No. 4, pp. 1335-1374.

Bouis, R. and R. Duval (2011), "Raising Potential Growth After the Crisis: A Quantitative Assessment of the Potential Gains from Various Structural Reforms in the OECD Area and Beyond", $O E C D$ Economics Department Working Papers, No. 835.

Bourlès, R. et al. (2010), "Do Product Market Regulations in Upstream Sectors Curb Productivity Growth: Panel Data Evidence for OECD Countries", OECD Economics Department Working Papers, No. 791 .

Broda, C. and J. Romalis (2009), “The Welfare Implications of Rising Price Dispersion”, mimeo, University of Chicago.

Buligescu, B. et al. (2008), "Panel estimates for the wage penalty for maternal leave", Oxford Economic Papers, Vol. 61, pp. i35-i55.

Burniaux, J., F. Padrini and N. Brandt (2006), "Labour Market Performance, Income Inequality and Poverty in OECD Countries", OECD Economics Department Working Papers, No. 500.

Bustos, P. (2007), "The Impact of Trade on Technology and Skill Upgrading: Evidence from Argentina", mimeo, CREI and Universitat Pompeu Fabra.

Caldéron, Chong, A. And R. Valdés, (2005), "Labor Market Regulations and Income Inequality: Evidence for a panel of Countries", in: J.E. Restrepo et al. (eds.), Labor Markets and Institutions, Chapter 7, pp. 221-279, Central Bank of Chile.

Calmfors, L. and J. Driffill (1988), "Bargaining Structure, Corporatism and Macroeconomic Performance", Economic Policy, No. 6, pp. 13-61.

Causa, O. and S. Jean (2007), "Integration of Immigrants in OECD Countries: Do Policies Matter?", OECD Economics Department Working Papers, No. 564.

Checchi, D. (2000), “Does Educational Achievement Help to Explain Income Inequality?”, UNU/WIDER Working Papers, No. 208.

Checchi, D. and C. Garcia-Peñalosa (2008), "Labour Market Institutions and Income Inequality”, Economic Policy, Vol. 23, No. 56, pp. 601-649.

Checchi, D. and C. Garcia-Peñalosa (2010), "Labour Market Institutions and the Personal Distribution of Income in the OECD”, Economica, Vol. 77, No. 307, pp. 413-450. 
Chetty, R. et al. (2011), "How Does Your Kindergarten Classroom Affect Your Earnings? Evidence from Project Star", Quarterly Journal of Economics, Vol. 126, No. 4.

Cohen-Goldner, S. and M.D. Paserman (2004), “The Dynamic Impact of Immigration on Natives' Labor Market Outcomes: Evidence from Israel," IZA Discussion Papers, No. 1315.

Conway, P. et. al. (2006), "Regulation, Competition and Productivity Convergence", OECD Economics Department Working Papers, No. 509.

D’Amuri, F. And G. Peri (2011), “Immigration, Jobs and Employment Protection: Evidence from Europe”, NBER Working Paper No. 17139.

Daveri, F. and G. Tabellini (2000), "Unemployment, Growth and Taxation in Industrial Countries", Economic Policy, Issue 30, pp. 47-104.

Davies, J. and I. Wooton (1992). "Income Inequality and International Migration", Economic Journal, Vol. 102, pp. 789-802.

De Gregorio, J. and J. Lee (2002), "Education and Income Inequality: New Evidence from Cross-Country Data", Review of Income and Wealth, Vol. 48, No. 3, pp. 395-416.

Dickens, R., S. Machin and A. Manning (1999), "The Effects of Minimum Wages on Employment: Theory and Evidence from Britain”, Journal of Labor Economics, Vol. 17, No. 1, pp. 1-22.

DiNardo, J., N.M. Fortin and T. Lemieux (1996), "Labor Market Institutions and the Distribution of Wages, 1973-1992: A Semiparametric Approach”, Econometrica, Vol. 64, No. 5, pp. 1001-1044.

Dinopoulos, E. and P. Segerstrom (1999), “A Schumpeterian Model of Protection and Relative Wages," American Economic Review, Vol. 89, No. 3, pp. 450-472.

Dupuy, A. and D. Fernández-Kranz (2011), "International Differences in the Family Gap in Pay: the Role of Labour Market Institutions", Applied Economics, Vol. 43, pp. 413-438.

Ebenstein, A. et al. (2009), "Estimating the Impact of Trade and Offshoring on American Workers Using the Current Population Surveys", NBER Working Papers, No. 15107, National Bureau of Economic Research.

Edwards, S. (1997), “Trade Policy, Growth, and Income Distribution?”, American Economic Review, Vol. 87, No. 2, pp. 205-210.

Egger, H. and U. Kreickemeier (2009), "Firm Heterogeneity and the Labor Market Effects of Trade Liberalization", International Economic Review, Vol. 50, No. 1, pp. 187-216.

Ehrhart, C. (2009), "The Effects of Inequality on Growth: A Survey of the Theoretical and Empirical Literature", ECINEQ Working Paper Series, No. 107, February.

Elmeskov, J., J. Martin and S. Scarpetta (1998), "Key Lessons for Labour Market Reforms: Evidence from OECD Countries’ Experiences”, Swedish Economic Policy Review, Vol. 5, No. 2.

Feenstra, R.C. (2008), "Offshoring in the Global Economy: Lecture 1: Microeconomic Structure; Lecture 2: Macroeconomic Implications," The Ohlin Lectures Presented at the Stockholm School of Economics 17-18 September. 
Feenstra, R.C. and G.H. Hanson (1996), "Foreign Investment, Outsourcing and Relative Wages", NBER Working Paper, No. 5121, National Bureau of Economic Research.

Fields G. (2001), Distribution and Development, A New Look at the Developing World, Russel Sage Foundation, New York, MIT Press.

Fiori, G., G. Nicoletti, S. Scarpetta and F. Schiantarelli (2007), "Employment Outcomes and the Interaction between Product and Labor Market Deregulation: Are they Substitutes or Complements?", IZA Discussion Papers, No. 2770, Institute for the Study of Labor (IZA).

Firpo, S., N. Fortin and T. Lemieux (2007a), “Unconditional Quantile Regression”, NBER Technical Working Paper, No. 339, July.

Firpo, S., N. Fortin and T. Lemieux (2007b), "Decomposing Wage Distributions using Recentered Influence Function Regression", mimeo, University of British Columbia, June.

Firpo, S., N. Fortin and T. Lemieux (2009), “Unconditional Quantile Regression”, Econometrica, Vol. 77, pp. 953-973, May.

Flabbi, L. (2011), "Gender Differentials in Education, Career, Choices and Labour Market Outcomes on a Sample of OECD Countries", mimeo.

Flanagan, R.J. (1999), "Macroeconomic Performance and Collective Bargaining: An International Perspective", Journal of Economic Literature, Vol. 37, pp. 1150-1175.

Fortin N.M., T. Lemieux, S. Firpo (2011), "Decomposition Methods in Economics", in: O. Ashenfelter and D. Card (eds.), Handbook of Labor Economics, Amsterdam: North-Holland, Elsevier, Vol. 4A, pp. 1-102.

Fournier, J.-M. and I. Koske (2012), "Less Income Inequality and More Growth - Are they Compatible? Part 7: The Drivers of Labour Earnings Inequality - An Analysis Based on Conditional and Unconditional Quantile Regressions”, OECD Economics Department Working Papers, No. 930.

Goldberg, P.K. and N. Pavcnik (2007), "Distributional Effects of Globalization in Developing Countries", Journal of Economic Literature, Vol. 45, No. 1, pp. 39-82.

Goos, M., A. Manning and A. Salomons (2009), "The Polarization of the European Labor Market", American Economic Review Papers and Proceedings, Vol. 99, No. 2, pp. 58-63.

Gosling, A. and S. Machin (1995), "Trade Unions and the Dispersion of Earnings in British Establishments, 1980-90”, Oxford Bulletin of Economics and Statistics, Vol. 57, No. 2, pp. 167-184.

Goux, D. and E. Maurin (2000), "Returns to Firm-Provided Training: Evidence from French Worker-Firm Matched Data", Labour Economics, Vol. 7, No. 1, pp. 1-19.

Griffith, R., R. Harrison and G. Macartney (2007), "Product Market Reforms, Labour Market Institutions and Unemployment", Economic Journal, Vol. 117.

Guadalupe, M. (2007), "Product Market Competition, Returns to Skill, and Wage Inequality", Journal of Labor Economics, Vol. 25, No. 3, pp. 439-474. 
Hanushek, E.A. and L. Wößmann (2006), "Does Educational Tracking Affect Performance and Inequality? Differences-In-Differences Evidence Across Countries", Economic Journal, Vol. 116, pp. C63-C76.

Harrison, A., J. McLaren and M.S. McMillan, (2010), "Recent Findings on Trade and Inequality", NBER Working Papers, No. 16425, National Bureau of Economic Research.

Hartog, J., P.T. Pereira and J.A.C. Vieira (2001), "Changing Returns to Education in Portugal during the 1980s and early 1990s: OLS and Quantile Regression Estimators", Applied Economics, Vol. 33, pp. 1021-1037.

Heller, D.E. (1999), "The Effects of Tuition and State Financial Aid on Public College Enrollment", Review of Higher Education, Vol. 23, No. 1, pp. 65-89.

Helpman, E., O. Itskhoki and S.J. Redding (2010), "Inequality and Unemployment in a Global Economy", Econometrica, Vol. 78, No. 4, pp. 1239-1283.

Hoxby, C.M. (2008), "School Spending, Income, and Inequality: The Efficient Redistribution Hypothesis", mimeo, Stanford University.

Jean, S. and J. Jiménez (2011), "The Unemployment Impact of Immigration in OECD Countries", European Journal of Political Economy, Vol. 27, No. 2, pp. 241-256.

Jean, S., O. Causa, M. Jiménez and I. Wanner (2010), "Migration and Labour Market Outcomes in OECD Countries", OECD Economic Studies, Vol. 2010, OECD Publishing.

Joumard, I., M. Pisu and D. Bloch (2012), "Less Income Inequality and More Growth - Are they Compatible? Part 3: Income Redistribution via Taxes and Transfers across OECD Countries", OECD Economics Department Working Papers, No. 926.

Kahn, L. (2000), "Wage Inequality, Collective Bargaining and Relative Employment from 1985 to 1994: Evidence from 15 OECD Countries", Review of Economics and Statistics, Vol. 82, No. 4, pp. 564-579.

Katz, L.F. and K.M. Murphy (1992), "Changes in Relative Wages, 1963-1987: Supply and Demand Factors", Quarterly Journal of Economics, Vol. 107, No. 1, pp. 35-78.

Killingsworth, M. (1987), "Heterogeneous Preferences, Compensating Wage Differentials and Comparable Worth", Quarterly Journal of Economics, Vol. 102, pp. 727-742.

Kluve, J. and C.M. Schmidt (2002), "Can Training and Employment Subsidies Combat European Unemployment?”, Economic Policy, Vol. 17, No. 35, pp. 409-448.

Knight, J.B. and R.H. Sabot (1983), "Educational Expansion and the Kuznets Effect", American Economic Review, Vol. 73, No. 5, pp. 1132-1136.

Koeniger, W., M. Leonardi and L. Nunziata (2007), "Labor Market Institutions and Wage Inequality", Industrial and Labor Relations Review, Vol. 60. No. 3, pp. 340-356.

Koenker, R. and G. Basset (1978), "Quantile Regression”, Econometrica, Vol. 46, pp. 33-50, January.

Lee, D.S. (1999), "Wage Inequality in the US during the 1980s: Rising Dispersion or Falling Minimum Wage?", Quarterly Journal of Economics, Vol. 114, No. 3, pp. 941-1023. 
Lemieux, T. (2006a), "Increasing Residual Wage Inequality: Composition Effects, Noisy Data, or Rising Demand for Skill?", American Economic Review, Vol. 96, No. 3, pp. 461-498.

Lemieux, T. (2006b), "Postsecondary Education and Increasing Wage Inequality", American Economic Review, Vol. 96, No. 2, pp. 195-199.

Lemieux, T., W.B. MacLeod and D. Parent (2009), "Performance Pay and Wage Inequality", Quarterly Journal of Economics, Vol. 144, Issue 1.

Leseman, P.P.M. (2002), "Early Childhood Education and Care for Children from Low-Income or Minority Backgrounds", a paper for discussion at the OECD Oslo Workshop, 6-7 June, 2002.

Leuven, E. and H. Oosterbeek (2002), "A new Method to Estimate the Returns to Work-related Training", mimeo, Department of Economics, University of Amsterdam.

Liebig, T. and A. Sousa-Poza (2004), "Migration, Self-Selection and Income Inequality: An International Analysis", Kyklos, Vol. 57, 125-146.

Liu, R. and D. Trefler (2008), "Much Ado About Nothing: American Jobs and The Rise of Service Outsourcing to China and India", NBER Working Papers, No. 14061, National Bureau of Economic Research.

Livanos, I., Ç. Yalkin and I. Nuñez, (2009), "Gender Employment Discrimination: Greece and the United Kingdom”, International Journal of Manpower, Vol. 30, pp. 815-834.

Machado, J.A.F. and J. Mata (2001), "Earning Functions in Portugal 1982-1994: Evidence from Quantile Regressions", Empirical Economics, Vol. 26, No. 1, pp. 115-134.

Machin, S. (1997), "The Decline of Labour Market Institutions and the Rise in Wage Inequality in Britain”, European Economic Review, Vol. 41, No. 3-5, pp. 647-657.

Makdissi, P. and M. Yazbeck (2011), "Assessing the Redistributive Impact of Higher Education Tuition Fees Reforms: the Case of Québec”, Applied Economics Letters, Vol. 18, pp. 143-147.

Martin, J.P. and D. Grubb (2001), "What Works and for Whom: A Review of OECD Countries' Experiences with Active Labour Market Policies", Swedish Economic Policy Review, Vol. 8, No. 2, pp. 9-56.

McCabe, B. and E. Smyth (2000), "The Educational Situation of Disadvantaged Children", in: I. Nicaise (ed.), The Right to Learn: Educational Strategies for Socially Excluded Youth in Europe, The Policy Press, Bristol.

Mullis, I. et al. (2003), "PIRLS 2001 International Report: IEA's Study of Reading Literacy Achievement in Primary Schools in 35 Countries", International Study Center, Lynch School of Education, Boston College.

Murtin, F., de Serres, A. and A. Hijzen (2012), “The Ins and Outs of Unemployment: The role of Labour Market Institutions”, OECD Economics Department Working Paper, forthcoming.

Neary, J.P. (2003), "Presidential Address: Globalization and Market Structure", Journal of the European Economic Association, Vol. 1, No. 2-3, pp. 245-271. 
Neumark, D. and W. Wascher (2007), "Minimum Wages and Employment", IZA Discussion Papers, No. 2570.

Nickell, S., L. Nunziata and W. Ochel (2005), "Unemployment in the OECD Since the 1960s: What Do We Know?”, Economic Journal, Vol. 115.

Nicoletti, G. et al. (2001), "Product and Labour Markets Interactions in OECD Countries", OECD Economics Department Working Papers, No. 312.

Nicoletti, G. and S. Scarpetta (2005), "Product Market Reforms and Employment in OECD Countries", OECD Economics Department Working Papers, No. 472.

Nunziata, L. (2002), "Unemployment, Labour Market Institutions and Shocks", Nuffield College Working Papers in Economics, 2002-W16.

OECD (2004), OECD Employment Outlook, OECD Publishing.

OECD (2006a), Starting Strong II: Early Childhood Education and Care, OECD Publishing.

OECD (2006b), OECD Employment Outlook 2006: Boosting Jobs and Incomes, OECD Publishing.

OECD (2007), No More Failures: Ten Steps to Equity in Education, OECD Publishing.

OECD (2010a), Education at a Glance 2010, OECD Publishing.

OECD (2010b), The High Cost of Low Educational Performance: The Long-run Economic Impact of Improving PISA Outcomes, OECD Publishing.

OECD (2011a), Divided We Stand: Why Inequality Keeps Rising, OECD Publishing.

OECD (2011b), Report on the Gender Initiative: Gender Equality in Education, Employment and Entrepreneurship, meeting of the OECD Council at Ministerial Level, 25-26 May 2011, Paris.

Orrenius, P.M. and M. Zavodny (2007), "Does Immigration Affect Wages? A Look at the OccupationLevel Evidence", Labour Economics, Vol. 14, No. 5, pp. 757-773.

Ottaviano, G. and G. Peri (2012), "Rethinking the Effects of Immigration on Wages", Journal of the European Economic Association, forthcoming.

Polachek, S. (1981), "Occupational Self-selection: A Human Capital Approach to Sex Differences in Occupational Structure", Review of Economics and Statistics, Vol. 63, pp. 60-69.

Ponthieux, S. and D. Meurs (2005), "The Gender Wage Gap in Europe: Women, Men and the Public Sector", INSEE, Direction des Statistiques Démographiques et Sociales Working Paper No. F0502.

Richardson, J.D. (1995), "Income Inequality and Trade: How to Think, What to Conclude", Journal of Economic Perspectives, Vol. 9, pp. 33-55.

Robinson, S. (1976), "Note on the U Hypothesis Relating Income Inequality and Economic Development", American Economic Review, Vol. 66, pp. 437-440. 
Sala-i-Martin, X., G. Doppelhofer and R.I. Miller (2004). "Determinants of Long-Term Growth: A Bayesian Averaging of Classical Estimates (BACE) Approach", American Economic Review, Vol. 94, pp. 813-835.

Santiago, P. et al. (2008), Tertiary Education for the Knowledge Society. Vol. 1: Special Features: Governance, Funding, Quality, OECD Publishing.

Steinhardt, M.F. (2011), "The Wage Impact of Immigration in Germany - New Evidence for Skill Groups and Occupations", The B.E. Journal of Economic Analysis and Policy, Vol. 11, No. 1, Article 31.

Stone, S. and R. Cavazos (2011), "Wage Implications of Trade Liberalisation: Evidence for Effective Policy Formation", OECD Trade Policy Working Papers No. 122.

Sylwester, K. (2002), “Can Education Expenditures Reduce Income Inequality?”, Economics of Education Review, Vol. 21.

Teulings, C.N. (2003), “The Contribution of Minimum Wages to Increasing Wage Inequality”, Economic Journal, Vol. 113, No. 490, pp. 801-833.

Tinbergen, J. (1974), “Substitution of Graduate by Other Labour”, Kyklos, Vol. 27, No. 2, pp. 217-226.

Tinbergen, J. (1975), "Substitution of Academically Trained by Other Manpower", Review of World Economics, Vol. 111, No. 2, pp. 466-476.

Vanhoudt, P. (1997), "Do Labor Market Policies and Growth Fundamentals Matter for Income Inequality in OECD Countries?", IMF - Staff Papers, Vol. 44, No. 3, pp. 356-373.

Wood, A. (1995), "How Trade Hurt Unskilled Workers”, Journal of Economic Perspectives, Vol. 9, No. 3, pp. 15-32. 
Table A1. Details on the data set used in the Bayesian model averaging analysis

\begin{tabular}{|c|c|c|}
\hline Title & Definition & Sources \\
\hline & Dependent variables & \\
\hline Earnings decile ratios & $\begin{array}{l}\text { Wage refers to gross weekly or monthly earnings of } \\
\text { full-time workers }\end{array}$ & OECD Earnings Database \\
\hline \multirow[t]{2}{*}{ Gini } & $\begin{array}{l}\text { Ratio of the area between the } 45 \text { degree line and } \\
\text { the Lorenz curve over the total area under the } 45 \\
\text { degree line }\end{array}$ & $\begin{array}{l}\text { UNU-WIDER World Income } \\
\text { Inequality Database, Version } \\
\text { 2.0c, May } 2008\end{array}$ \\
\hline & Macro variables & \\
\hline $\begin{array}{l}\text { Trade (as share of } \\
\text { GDP) }\end{array}$ & $\begin{array}{l}\text { Trade exposure (weighted average of import } \\
\text { penetration and export intensity) }\end{array}$ & $\begin{array}{l}\text { United Nations Conference on } \\
\text { trade and development } \\
\text { (UNCTAD) }\end{array}$ \\
\hline $\begin{array}{l}\text { Foreign assets (as } \\
\text { share of GDP) }\end{array}$ & $\begin{array}{l}\text { Total assets= FDI assets+portfolio equity } \\
\text { assets+debt assets+derivatives assets+FX reserves } \\
\text { as a share of GDP }\end{array}$ & $\begin{array}{l}\text { Updated and extended version } \\
\text { of the External Wealth of } \\
\text { Nations Mark II Database } \\
\text { developed by Lane and Milesi- } \\
\text { Ferretti (2007) }\end{array}$ \\
\hline $\begin{array}{l}\text { Foreign liabilities (as } \\
\text { share of GDP) }\end{array}$ & $\begin{array}{l}\text { Total liabilities= FDI liabilities+portfolio equity } \\
\text { liabilities+debt liabilities+derivatives liabilities as a } \\
\text { share of GDP }\end{array}$ & $\begin{array}{l}\text { Updated and extended version } \\
\text { of the External Wealth of } \\
\text { Nations Mark II Database } \\
\text { developed by Lane and Milesi- } \\
\text { Ferretti (2007) }\end{array}$ \\
\hline \multirow[t]{2}{*}{$\begin{array}{l}\text { Patents (per } 1 \text { million } \\
\text { inhabitants) }\end{array}$} & $\begin{array}{l}\text { Patents (total patent applications to both European } \\
\text { patent office and the United States Patent and } \\
\text { Trademark Office), per million population. }\end{array}$ & $\begin{array}{l}\text { OECD Science and Technology } \\
\text { Indicators and OECD Patents } \\
\text { Database }\end{array}$ \\
\hline & Policy variables & \\
\hline $\begin{array}{l}\text { Share of population } \\
\text { with post-secondary } \\
\text { education }\end{array}$ & $\%$ of population has post-secondary education ${ }^{1}$ & $\begin{array}{l}\text { OECD Education at a Glance } \\
\text { and Barro and Lee (2000) } \\
\text { dataset }\end{array}$ \\
\hline $\begin{array}{l}\text { Employment } \\
\text { protection legislation }\end{array}$ & Index scale of $0-6$ from least to more restrictive & $\begin{array}{l}\text { OECD, Employment Outlook } \\
\text { Database }\end{array}$ \\
\hline Union density (in \%) & $\%$ of employees who are members of a trade-union & $\begin{array}{l}\text { OECD, Employment Outlook } \\
\text { Database }\end{array}$ \\
\hline $\begin{array}{l}\text { Unemployment } \\
\text { benefit replacement } \\
\text { rate }\end{array}$ & $\begin{array}{l}\text { Average gross unemployment benefit replacement } \\
\text { rate }\end{array}$ & $\begin{array}{l}\text { OECD, Benefits and Wages } \\
\text { Database }\end{array}$ \\
\hline $\begin{array}{l}\text { Minimum wage (as } \\
\text { ratio to median wage) }\end{array}$ & Minimum wage relative to median of full time worker & $\begin{array}{l}\text { OECD, Employment Outlook } \\
\text { Database }\end{array}$ \\
\hline Tax wedge (in \%) & $\begin{array}{l}\text { Tax wedges are calculated by expressing the sum } \\
\text { of personal income tax as a percentage of labour } \\
\text { costs. The reference rates are for a single person } \\
\text { without children at } 100 \% \text { of the average level. }\end{array}$ & OECD, Taxing Wages \\
\hline $\begin{array}{l}\text { Product market } \\
\text { regulation indicator }\end{array}$ & $\begin{array}{l}\text { Indicators of regulation in seven non manufacturing } \\
\text { sectors (telecoms, electricity, gas, post, rail, air } \\
\text { passenger transport, and road freight). } \\
\text { Index scale of } 0-6 \text { from least to more restrictive. }\end{array}$ & $\begin{array}{l}\text { OECD, Product Market } \\
\text { Regulation Database }\end{array}$ \\
\hline Financial reform index & $\begin{array}{l}\text { Financial Reform Index, } 0 \text { to } 21 \text {, sum of seven } \\
\text { components; higher number means more } \\
\text { liberal/developed financial system. }\end{array}$ & Abiad et al. (2010) \\
\hline
\end{tabular}


Table A1. Details on the data set used in the Bayesian model averaging analysis, continued

\begin{tabular}{|c|c|c|}
\hline \multicolumn{3}{|c|}{ Control variables } \\
\hline $\begin{array}{l}\text { Sectoral employment } \\
\text { shares }\end{array}$ & $\begin{array}{l}\% \text { of employment in agriculture and industry as a } \\
\text { share of total employment. }\end{array}$ & OECD, Employment Database \\
\hline $\begin{array}{l}\text { Female employment } \\
\text { share }\end{array}$ & $\begin{array}{l}\% \text { of female employment as a share of total } \\
\text { employment. }\end{array}$ & OECD, Employment Database \\
\hline Output gap & $\begin{array}{l}\text { Deviations of actual GDP from potential GDP as a } \\
\text { percentage of potential GDP }\end{array}$ & $\begin{array}{l}\text { OECD, Economic Outlook } \\
\text { Database }\end{array}$ \\
\hline \multicolumn{3}{|c|}{$\begin{array}{l}\text { 1. Data for } 1980,85,90,95 \text { and } 2000 \text { are drawn from the Barro and Lee (2000) dataset, and for the years } 2001-08 \text { data are from } \\
\text { OECD Education at a Glance. For the years between } 1985 \text { and } 2000 \text {, data are interpolated linearly. }\end{array}$} \\
\hline \multicolumn{3}{|c|}{$\begin{array}{l}\text { Source: Lane, P.R. and G.M. Milesi-Ferretti (2007), "The External Wealth of Nations Mark II", Journal of International Econom } \\
\text { Vol. 73, pp. 223-250; Barro, R.J. and J.-W. Lee (2000), "International Data on Educational Attainment: Updates and Implications", } \\
\text { Working Paper No. 42; Abiad, A., E. Detragiache and T. Tressel (2010), "A New Database of Financial Reforms", IMF Staff Pap } \\
\text { Vol. 57, pp. 281-302. }\end{array}$} \\
\hline
\end{tabular}

Figure A1. Policies influencing the distribution of labour income

Australia

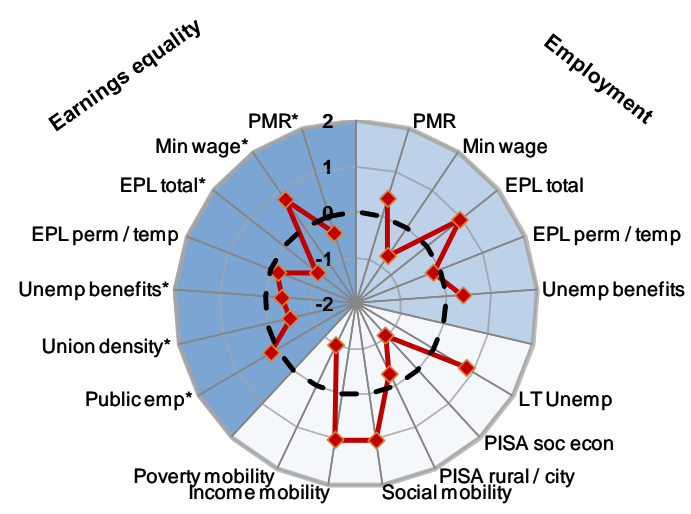

Equality of opportunity

Belgium

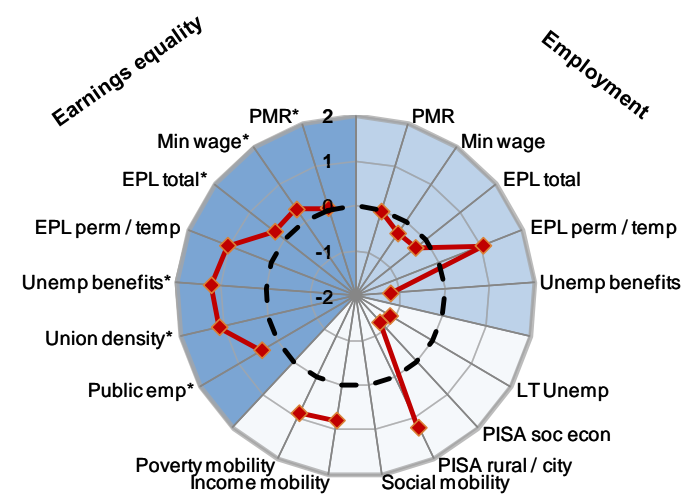

Equality of opportunity
Austria

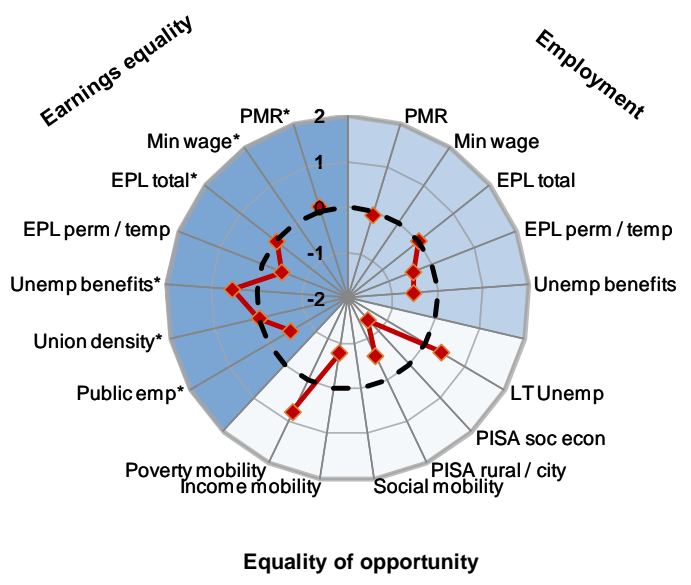

Canada

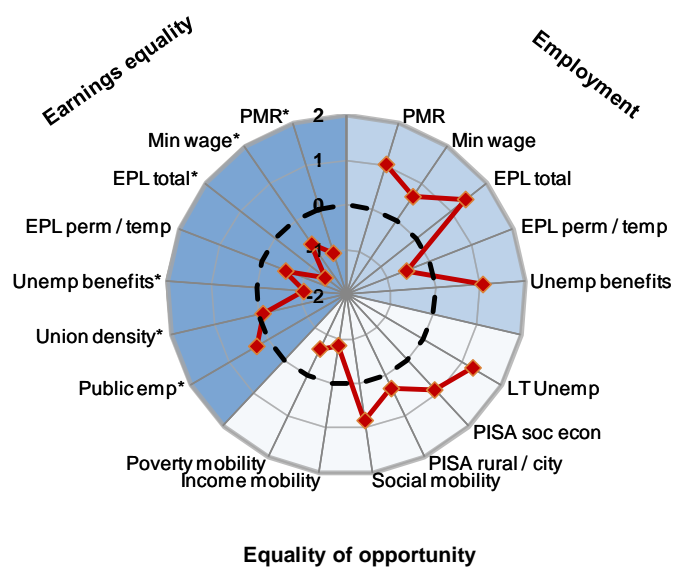


Figure A1. Policies influencing the distribution of labour income, continued

Chile

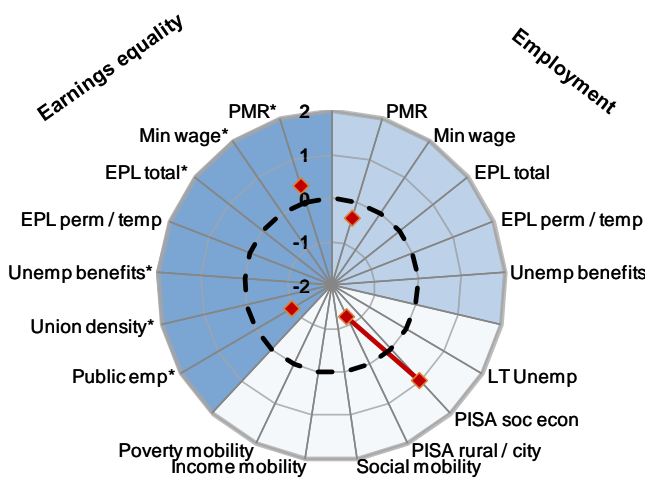

Equality of opportunity

Denmark

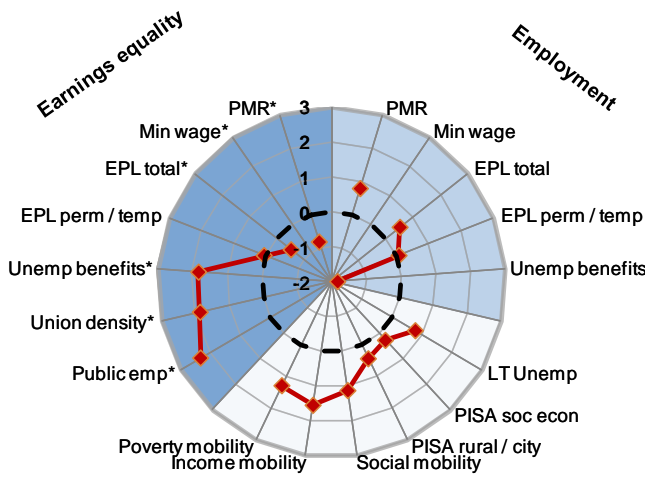

Equality of opportunity

Finland

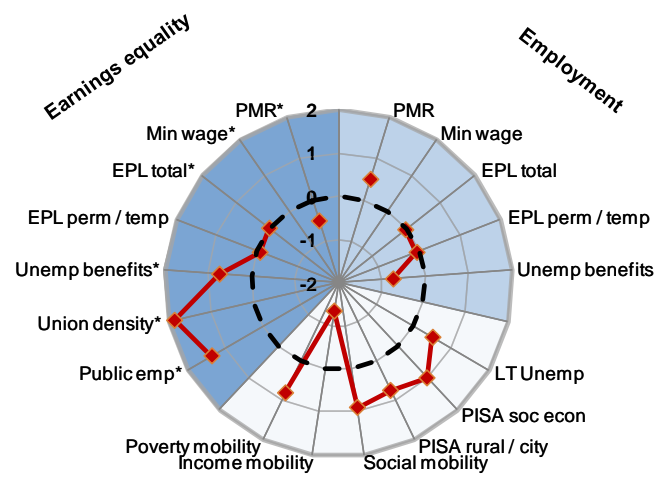

Equality of opportunity
Czech Republic

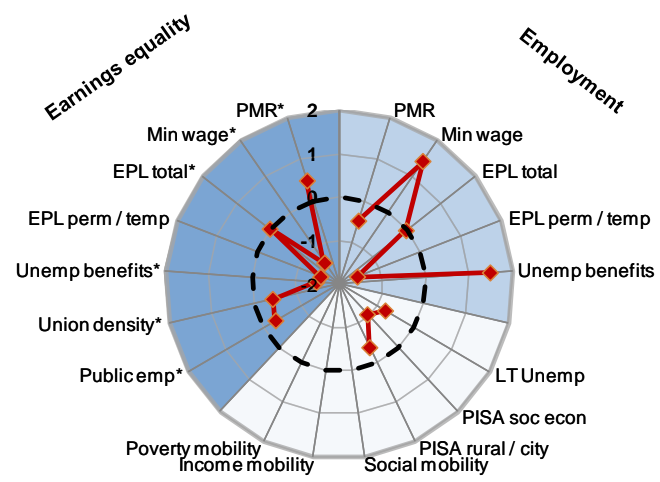

Equality of opportunity

Estonia

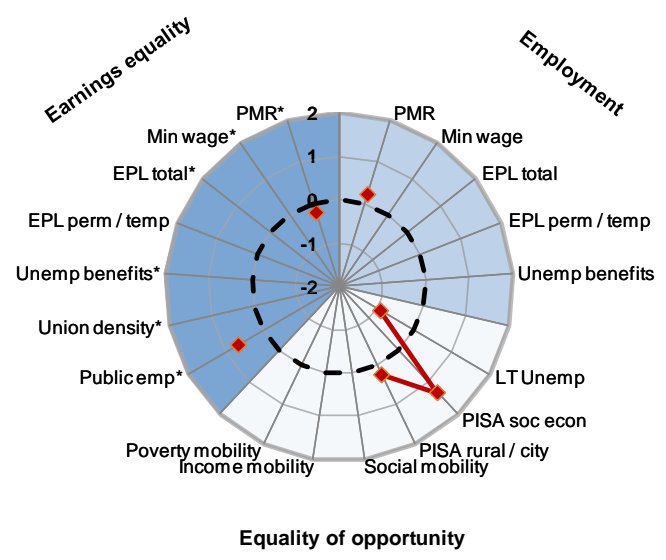

France

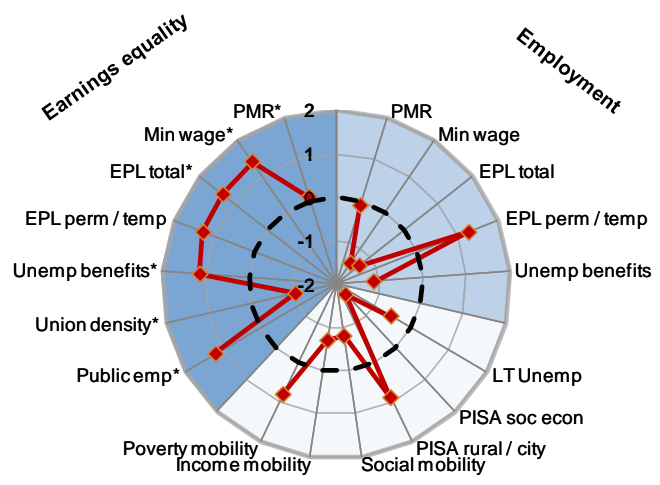

Equality of opportunity 
ECO/WKP(2012)2

Figure A1. Policies influencing the distribution of labour income, continued

Germany

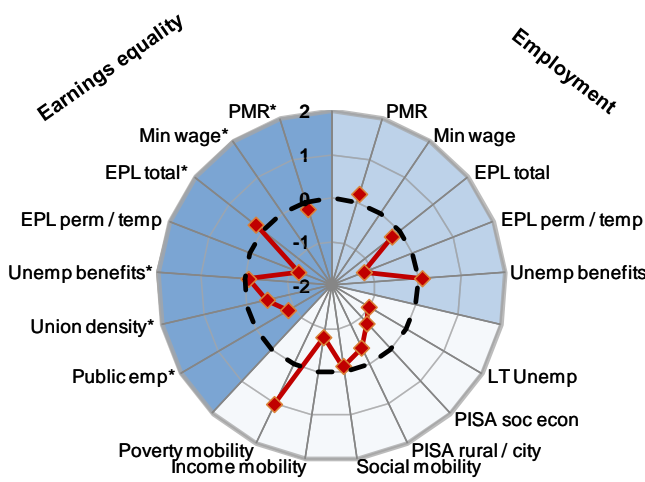

Equality of opportunity

Hungary

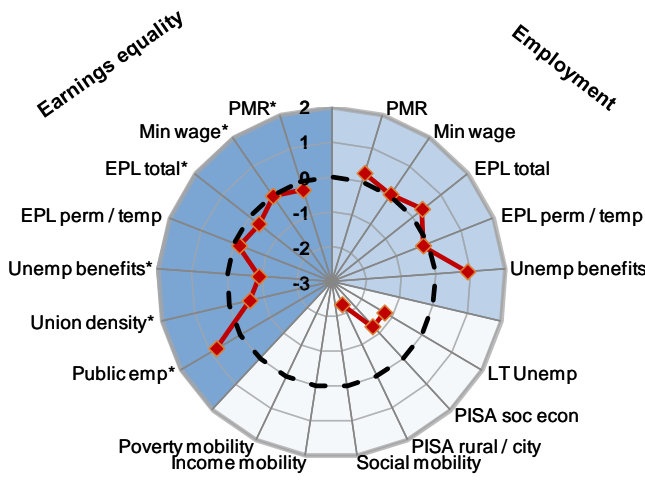

Equality of opportunity

Ireland

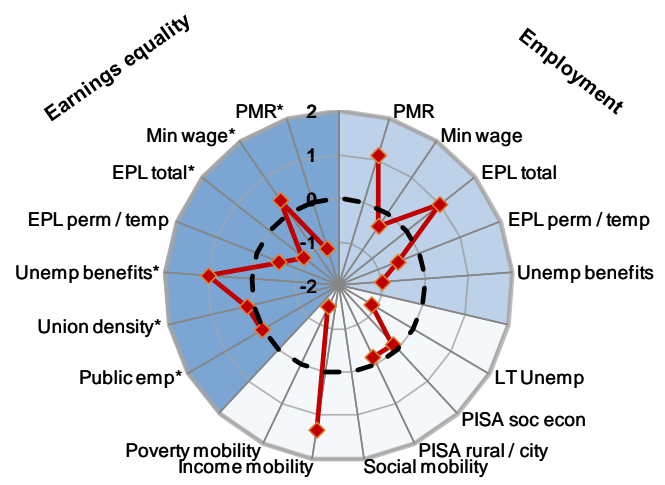

Equality of opportunity
Greece

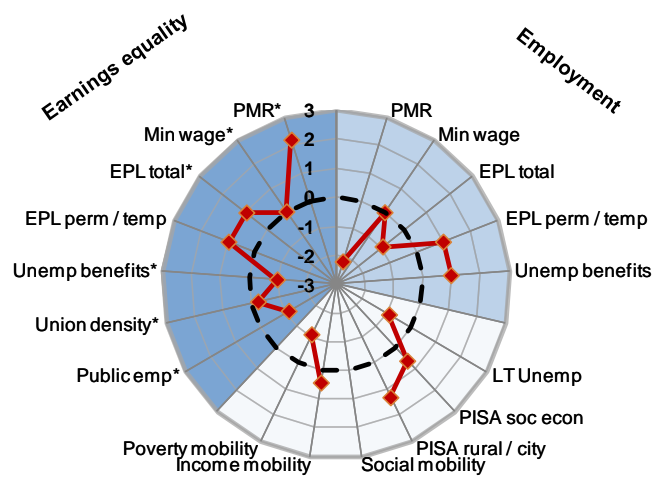

Equality of opportunity

Iceland

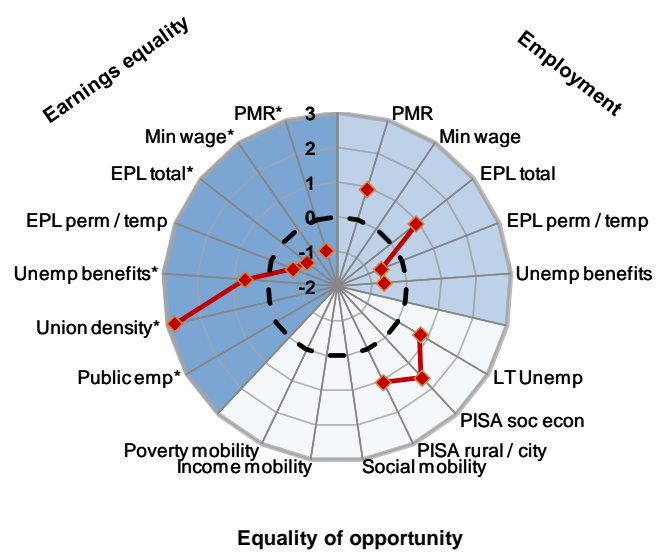

Israel

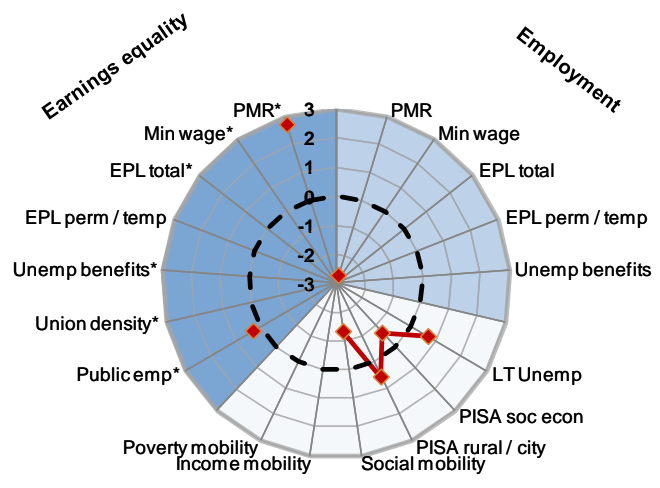

Equality of opportunity 
Figure A1. Policies influencing the distribution of labour income, continued

Italy

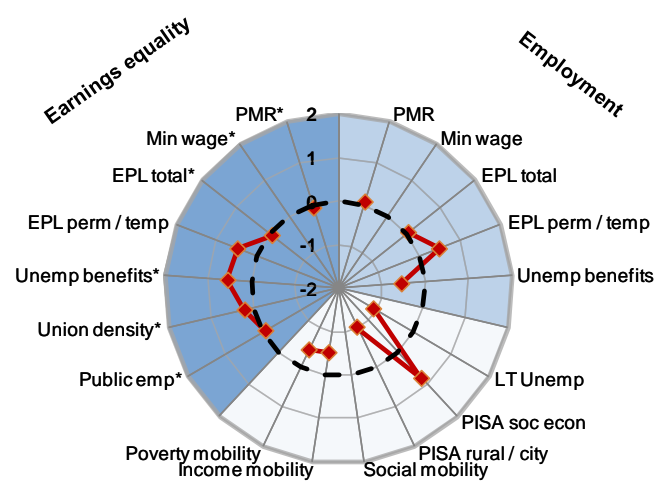

Equality of opportunity

Korea

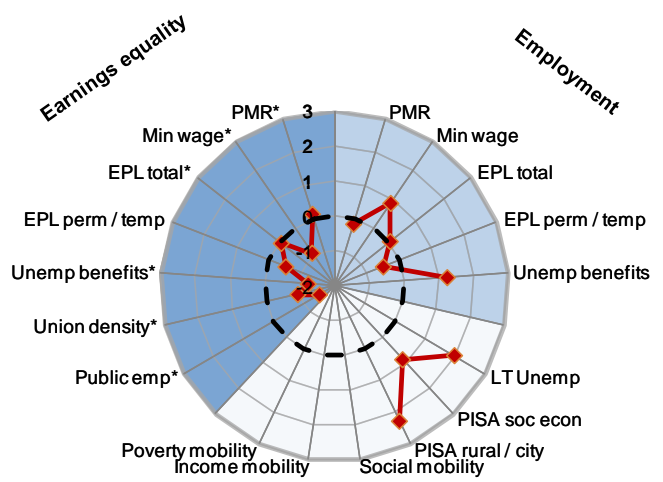

Equality of opportunity

Mexico

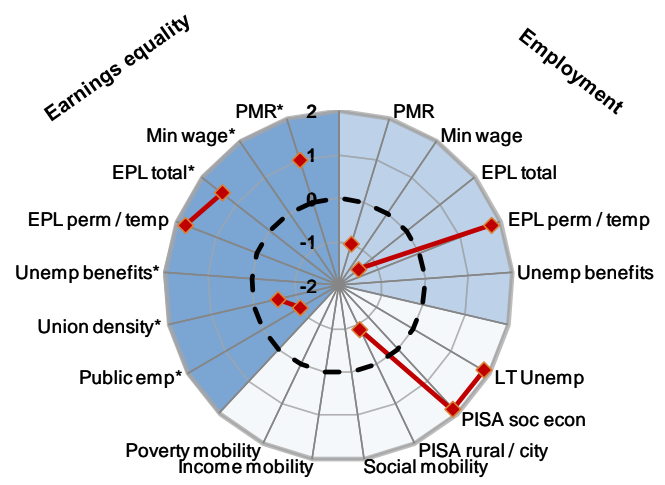

Equality of opportunity
Japan

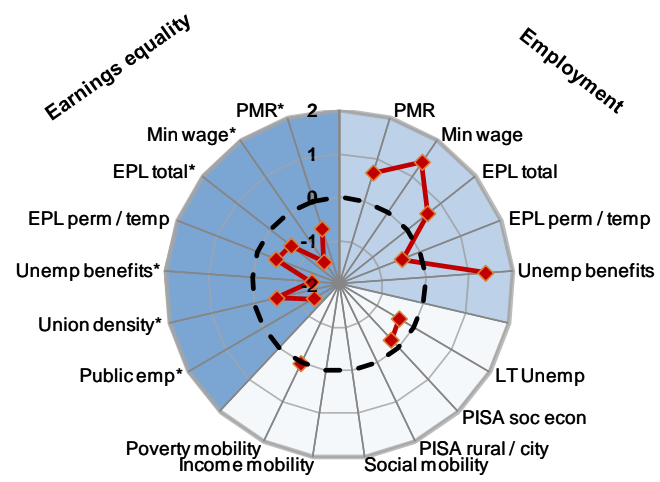

Equality of opportunity

Luxembourg

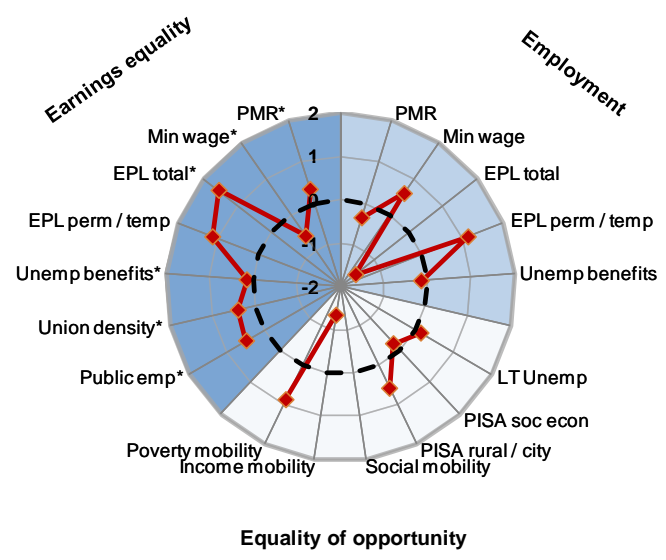

Netherlands

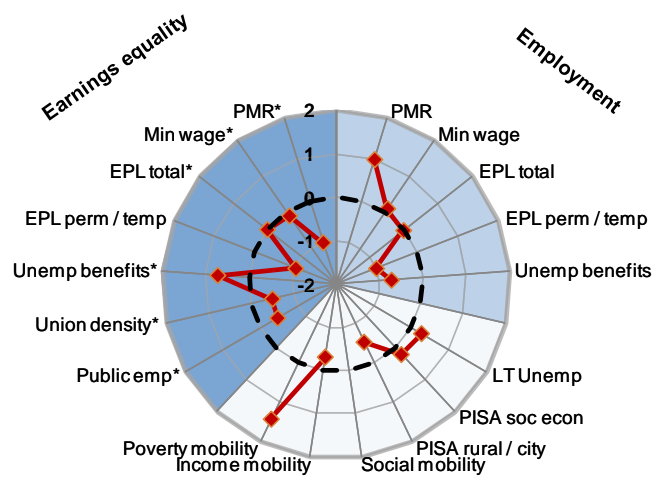

Equality of opportunity 
Figure A1. Policies influencing the distribution of labour income, continued

New Zealand

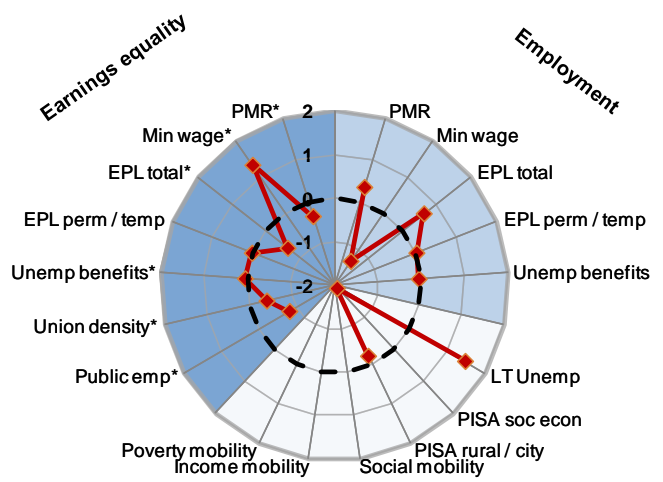

Equality of opportunity

Poland

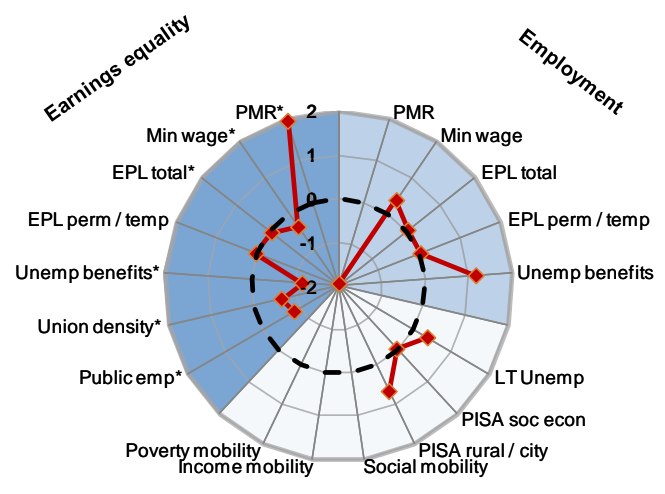

Equality of opportunity

Slovak Republic

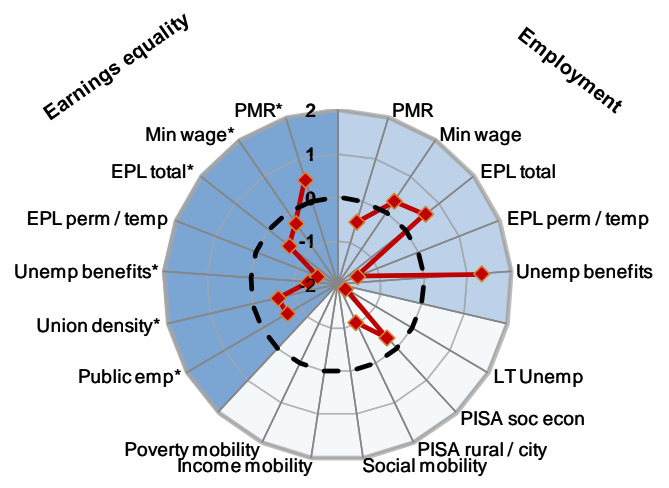

Equality of opportunity
Norway

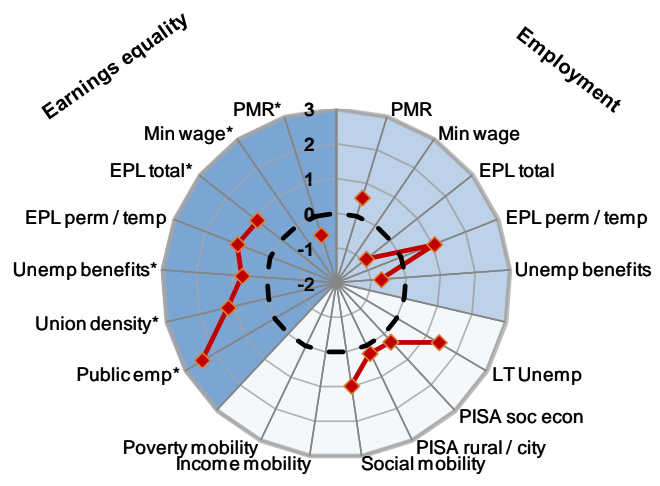

Equality of opportunity

Portugal

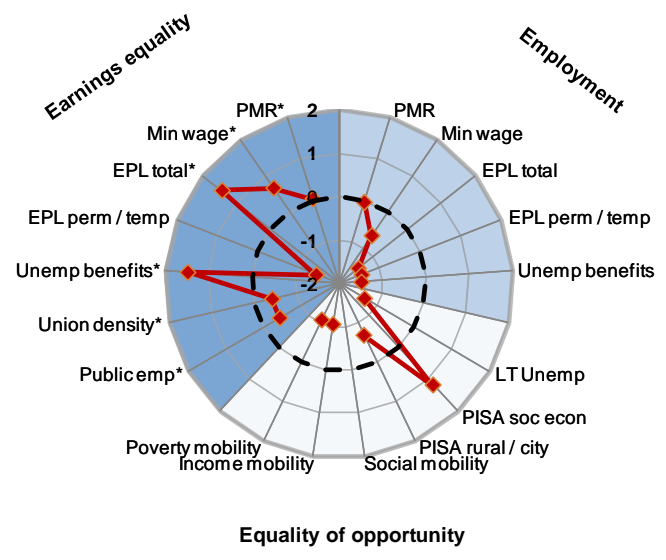

Slovenia

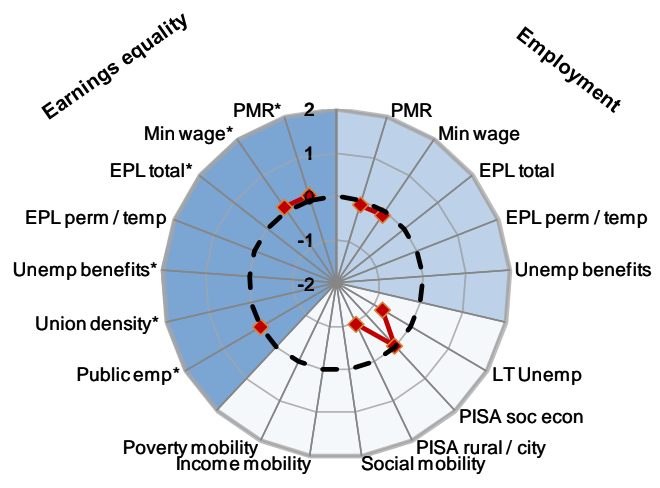

Equality of opportunity 
Figure A1. Policies influencing the distribution of labour income, continued

Spain

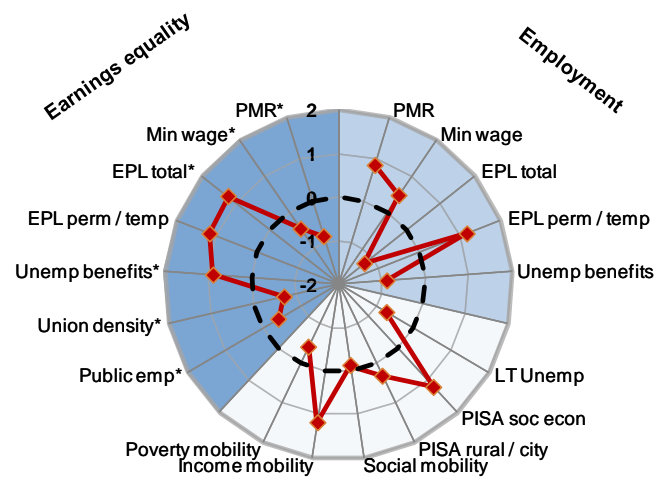

Equality of opportunity

Switzerland

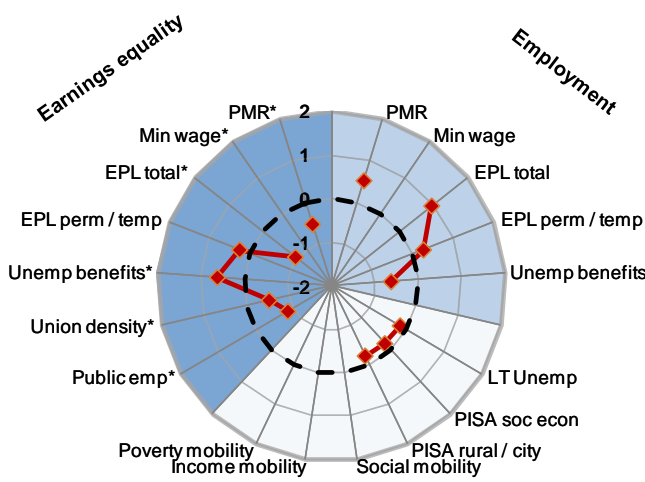

Equality of opportunity

United Kingdom

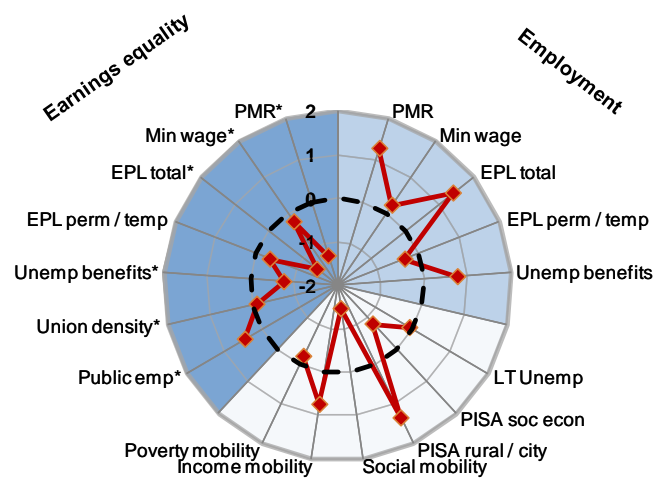

Equality of opportunity
Sweden

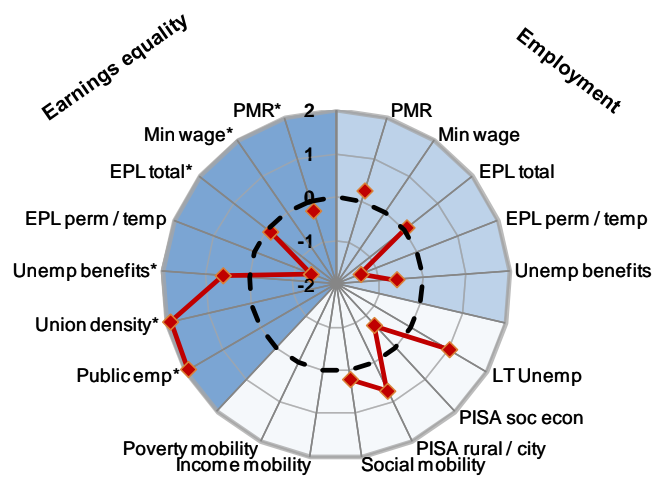

Equality of opportunity

Turkey

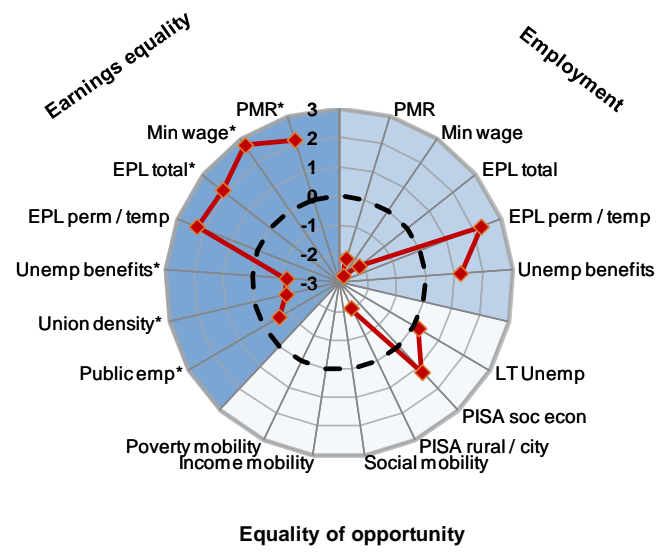

United States

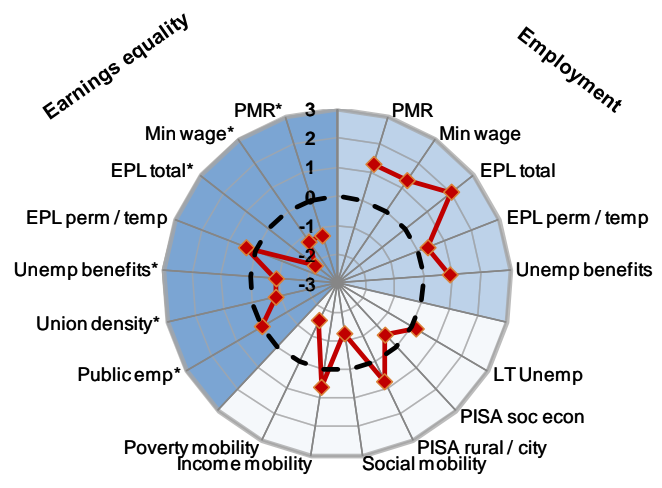

Equality of opportunity 
ECO/WKP(2012)2

Figure A1. Policies influencing the distribution of labour income, continued

\section{Legend:}

$\mathrm{PMR}=$ Economy-wide product market regulation

Min wage $=$ Ratio of minimum to median wage

Union density = Share of workers affiliated to a trade union

$\mathrm{EPL}=$ Employment Protection Legislation

EPL perm/temp = Difference in EPL between permanent and temporary workers

Unemp benefits $=$ Average gross unemployment benefit replacement rate

Public emp = Employment in general government and public corporations as \% of total labour force

LT Unemp = Long-term unemployed as share of total unemployed

PISA soc econ = Average reading score point difference associated with socio-economic background

$\mathrm{PISA}$ rural/city = Reading performance, difference between students attending city schools vs. rural/village schools

Social mobility = Intergenerational earnings elasticity

Income mobility = Average share of people staying within HDI quintile (average of all quintiles)

Poverty mobility = Share of people experiencing persistent poverty

Note: The dotted line represents the OECD average, the solid line and diamond markers represent the country shown. Where the solid line falls outside the OECD average, this implies better results than the OECD average with regard to the three policy goals shown. The series with asterisks have been inversed to take into account the relationship between these policy variables and the policy goal. The indicators are presented in standard deviation units. 


\section{WORKING PAPERS}

The full series of Economics Department Working Papers can be consulted at www.oecd.org/eco/workingpapers/

924. Less income inequality and more growth - Are they compatible?

Part 1. Mapping income inequality across the OECD

(January 2012) by Peter Hoeller, Isabelle Joumard, Mauro Pisu and Debbie Bloch

923. Current issues in managing government debt and assets

(December 2011) by Eckhard Wurzel and Lukasz Rawdanowicz

922. Public spending efficiency in the Czech Republic: fiscal policy framework and the main spending areas of pensions and healthcare

(December 2011) by Zuzana Smidova

921. Exploring determinants of subjective wellbeing in OECD countries - evidence from the World Value Survey

(December 2011) by Sarah Fleche, Conal Smith and Piritta Sorsa

920. Russia: progress in structural reform and framework conditions

(December 2011) by Yana Vaziakova, Geoff Barnard and Tatiana Lysenko

919. Explaining the interest-rate-growth differential underlying government debt dynamics

(December 2011) by David Turner and Francesca Spinelli

918. Reassessing the NAIRUs after the crisis

(December 2011) by Stéphanie Guichard and Elena Rusticelli

917. Employment protection legislation and plant-level productivity in India

(December 2011) by Sean Dougherty, Verónica Frisancho Robles and Kala Krishna

916. Systemically important banks and capital regulation challenge

(December 2011) by Patrick Slovik

915. Improving educational outcomes in Slovenia

(December 2011) by Mehmet Eris

914. Addressing challenges in the energy sector in Israel

(December 2011) by Philip Hemmings

913. Issues in private sector finance in Israel

(December 2011) by Philip Hemmings

912. How to improve the economic policy framework for the housing market in Israel

(December 2011) by Philip Hemmings

911. Fiscal Prospects and Reforms in India

(December 2011) by Richard Herd, Sam Hill and Vincent Koen 
910. Structural reforms to reduce unemployment and restore competitiveness in Ireland (December 2011) by Álvaro Pina

909. Getting back on track: restoring fiscal sustainability in Ireland (December 2011) by David Haugh

908. A welfare analysis of climate change mitigation policies (November 2011) by Alain de Serres and Fabrice Murtin

907. Overcoming the banking crisis in Ireland

(November 2011) by Muge Adalet McGowan

906. Macroeconomic and structural policies to further stabilise the Mexican economy (November 2011) by Cyrille Schwellnus

905. Reaping the benefits of a transition to greener growth in Slovakia (November 2011) by Caroline Klein

904. Fiscal reform for a stronger fairer and cleaner Mexican economy (October 2011) by Nicola Brandt and Rodrigo Paillacar

903. The demand for safe assets in emerging economies and global unbalances: new empirical evidence (October 2011) by Rudiger Ahrend and Cyrille Schwellnus

902. Drivers of systemic banking crises: the role of bank-balance-sheet contagion and financial account structure

(October 2011) by Rudiger Ahrend and Antoine Goujard

901. Explaining the appreciation of the Brazilian Real

(October 2011) by Annabelle Mourougane

900. Raising investment in Brazil

(October 2011) by Jens Arnold

899. Refining macroeconomic policies to sustain growth in Brazil

(October 2011) by Annabelle Mourougane

898. Promoting infrastructure development in Brazil

(October 2011) by Annabelle Mourougane and Mauro Pisu

897. Austria: public sector inefficiencies have become less affordable

(October 2011) by Karin Fischer, Rauf Gönenç and Robert Price

896. Informality in Mexico

(September 2011) by Nicola Brandt

895. Reforming Austria's highly regarded but costly health system

(September 2011) by Rauf Gönenç, Maria. M. Hofmarcher, Andreas Wörgötter 